\title{
2015 ESC/ERS Guidelines for the diagnosis and treatment of pulmonary hypertension
}

\author{
The Joint Task Force for the Diagnosis and Treatment of Pulmonary \\ Hypertension of the European Society of Cardiology (ESC) and the \\ European Respiratory Society (ERS) \\ Endorsed by: Association for European Paediatric and Congenital Cardiology \\ (AEPC), International Society for Heart and Lung Transplantation (ISHLT) \\ Nazzareno Galiè ${ }^{1}$ (ESC Chairperson), Marc Humbert ${ }^{2}$ (ERS Chairperson), \\ Jean-Luc Vachiery ${ }^{3}$, Simon Gibbs ${ }^{1}$, Irene Lang ${ }^{1}$, Adam Torbicki ${ }^{1}$, \\ Gérald Simonneau ${ }^{2}$, Andrew Peacock ${ }^{2}$, Anton Vonk Noordegraaf ${ }^{2}$, \\ Maurice Beghetti ${ }^{4}$, Ardeschir Ghofrani ${ }^{2}$, Miguel Angel Gomez Sanchez ${ }^{1}$, \\ Georg Hansmann ${ }^{4}$, Walter Klepetko ${ }^{3}$, Patrizio Lancellotti ${ }^{1}$, Marco Matucci ${ }^{5}$, \\ Theresa McDonagh ${ }^{1}$, Luc A. Pierard ${ }^{1}$, Pedro T. Trindade ${ }^{1}$, Maurizio Zompatori ${ }^{6}$ \\ and Marius Hoeper ${ }^{2}$
}

Affiliations: ${ }^{1}$ Representing the European Society of Cardiology. ${ }^{2}$ Representing the European Respiratory Society. ${ }^{3}$ Representing the International Society for Heart and Lung Transplantation. ${ }^{4}$ Representing the Association for European Paediatric and Congenital Cardiology. ${ }^{5}$ Representing the European League Against Rheumatism. ${ }^{6}$ Representing the European Society of Radiology. A full list of collaborators and document reviewers can be found in the Appendix.

\section{Correspondence:}

Nazzareno Galiè, Dept of Experimental, Diagnostic and Specialty Medicine-DIMES, University of Bologna, Via Massarenti 9, 40138 Bologna, Italy. E-mail: nazzareno.galiedunibo.it

Marc Humbert, Service de Pneumologie, Hôpital Bicêtre, Université Paris-Sud, Assistance Publique Hôpitaux de Paris, 78 rue du Général Leclerc, 94270 Le Kremlin-Bicêtre, France. E-mail: marc.humbertđaphp.fr

@ERSpublications

2015 ESC/ERS pulmonary hypertension guidelines incorporate changes and adaptations focusing on clinical management http://ow.ly/RiDLb

Editorial Comments in Eur Respir J 2015; 46: 879-882 [DOI: 10.1183/13993003.01177-2015].

This article has been revised according to the erratum published in the December 2015 issue of the European Respiratory Journal.

The content of these European Society of Cardiology (ESC) and European Respiratory Society (ERS) Guidelines has been published for personal and educational use only. No commercial use is authorized. No part of the ESC/ERS Guidelines may be translated or reproduced in any form without written permission from the ESC and/or ERS. Permission can be obtained upon submission of a written request to Oxford University Press, the publisher of the European Heart Journal or from the European Respiratory Society, the publisher of European Respiratory Journal and the party authorized to handle such permissions on behalf of the ESC and ERS.

This article is being published concurrently in the European Heart Journal (10.1093/eurheartj/ehv317) and the European Respiratory Journal (10.1183/13993003.01032-2015). The articles are identical except for minor stylistic and spelling differences in keeping with each journal's style. Either citation can be used when citing this article.

Conflict of interest: Disclosures can be found alongside the online version of this article at erj.ersjournals.com

Published on behalf of the European Society of Cardiology and European Respiratory Society. All rights reserved. ๑ 2015 European Society of Cardiology \& European Respiratory Society. 


\section{Table of Contents}

Abbreviation and acronyms

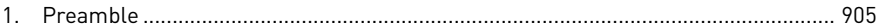

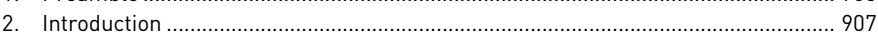

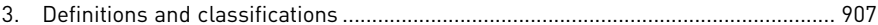

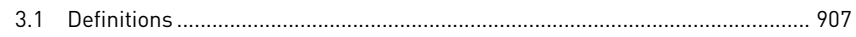

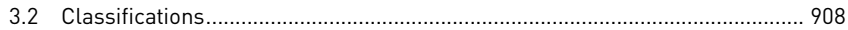

4. Epidemiology and genetics of pulmonary hypertension ....................................... 909

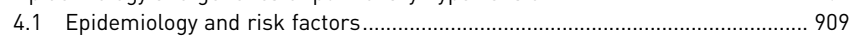

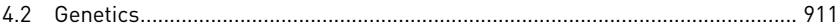

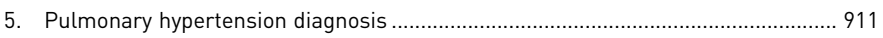

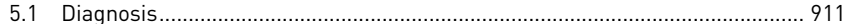

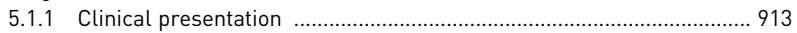

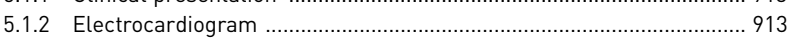

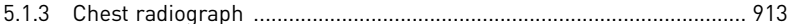

5.1.4 Pulmonary function tests and arterial blood gases .......................... 913

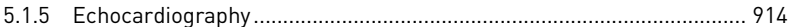

5.1.6 Ventilation/perfusion lung scan...................................................... 915

5.1.7 High-resolution computed tomography, contrast enhanced computed tomography, and pulmonary angiography ………............... 916

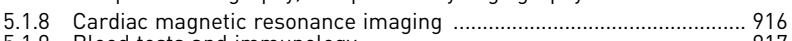

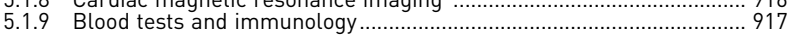

5.1.10 Abdominal ultrasound scan ..................................................... 917

5.1.11 Right heart catheterization and vasoreactivity ................................. 917

5.1.12 Genetic testing ........................................................................ 918

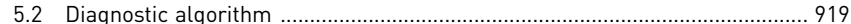

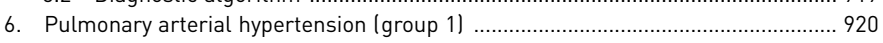

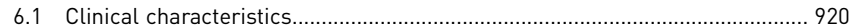

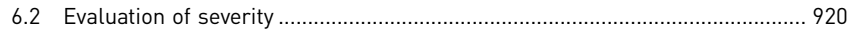

6.2.1 Clinical parameters, imaging and haemodynamics ......................... 920

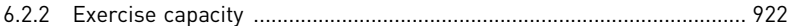

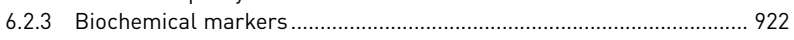

6.2.4 Comprehensive prognostic evaluation and risk

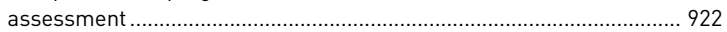

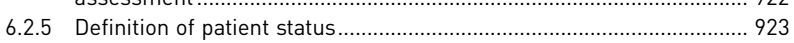

6.2.6 Treatment goals and follow-up strategy.............................................. 924

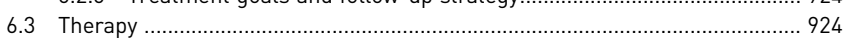

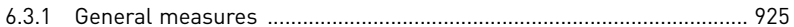

6.3.1.1 Physical activity and supervised rehabilitation ................................. 925

6.3.1.2 Pregnancy, birth control, and post-menopausal

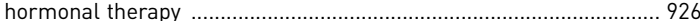

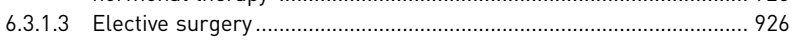

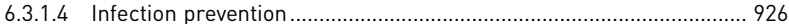

6.3.1.5 Psychosocial support ............................................................. 926

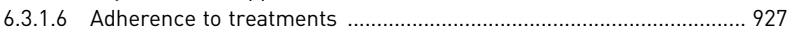

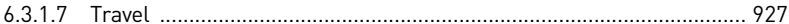

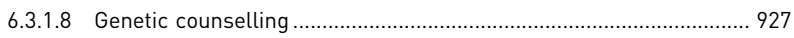

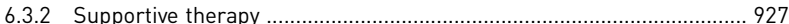

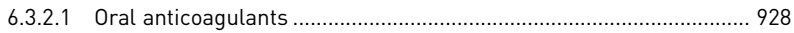

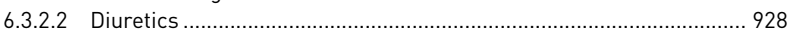

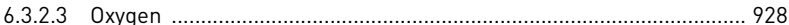

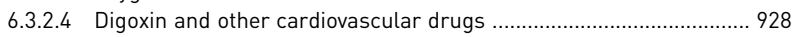

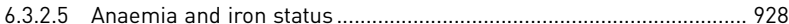

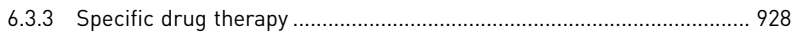

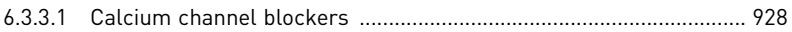

6.3.3.2 Endothelin receptor antagonists .................................................. 929

6.3.3.3 Phosphodiesterase type 5 inhibitors and guanylate cyclase

stimulators.
6.3.3.4 Prostacyclin analogues and prostacyclin receptor agonists ........... 931

6.3.3.5 Experimental compounds and strategies ...................................... 932

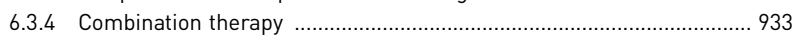

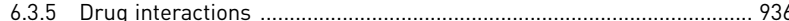

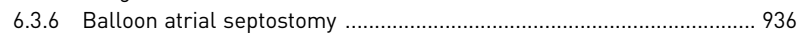

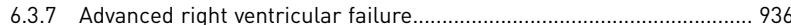

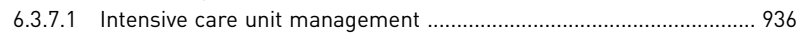

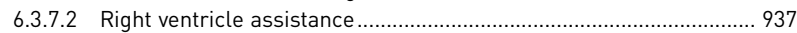

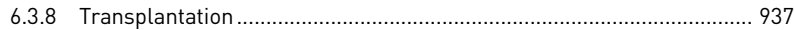

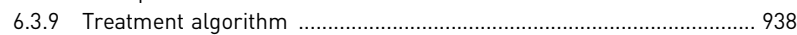

6.3.10 Diagnosis and treatment of pulmonary arterial hypertension complications ........................................................... 939

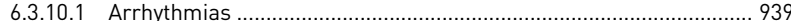

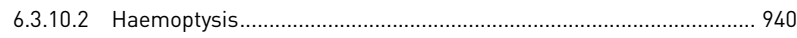

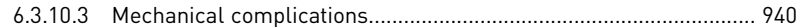

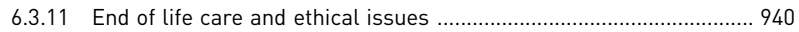

7. Specific pulmonary (arterial) hypertension subsets ........................................... 940

7.1 Paediatric pulmonary arterial hypertension .................................................. 940

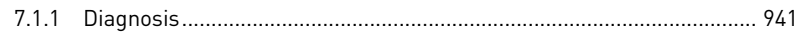

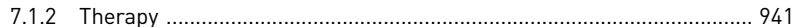

7.2 Pulmonary arterial hypertension associated with adult

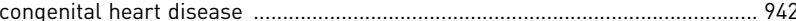

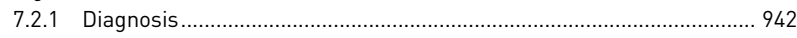

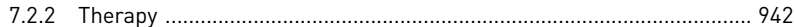

7.3 Pulmonary arterial hypertension associated with

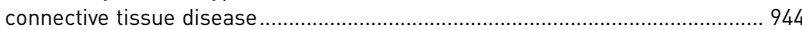

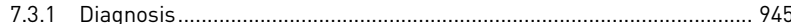

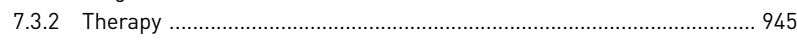

7.4 Pulmonary arterial hypertension associated with portal hypertension ............ 945

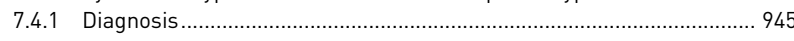

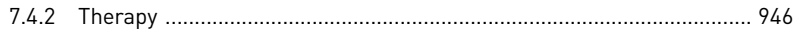

7.5 Pulmonary arterial hypertension associated with human

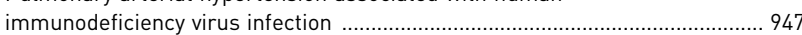

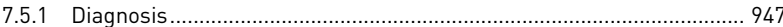

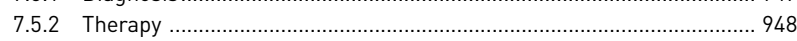

7.6 Pulmonary veno-occlusive disease and pulmonary

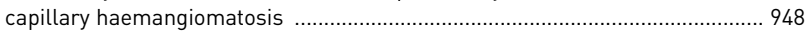

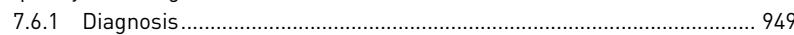

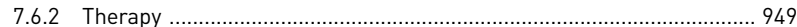

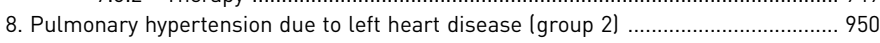

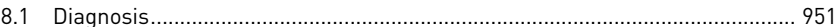

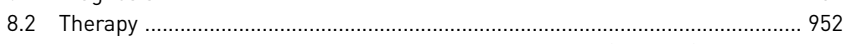

9. Pulmonary hypertension due to lung diseases and/or hypoxia (group 3) ................ 952

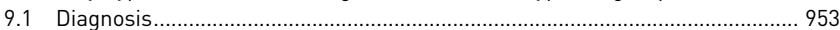

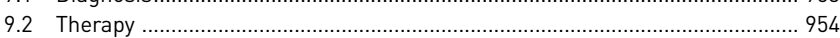

10. Chronic thromboembolic pulmonary hypertension (group 4) ............................... 954

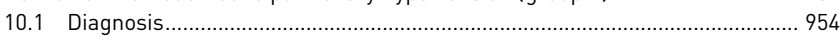

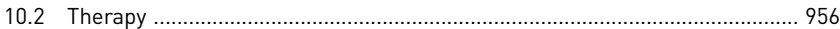

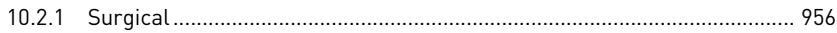

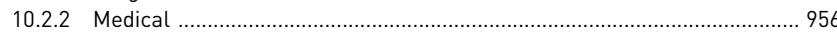

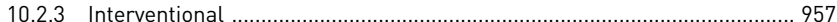

11. Pulmonary hypertension with unclear and/or

multifactorial mechanisms (group 5)

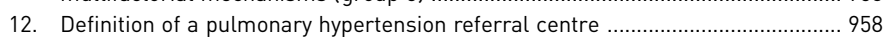

13. To do and not to do messages from the guidelines ........................................... 960

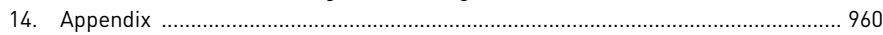

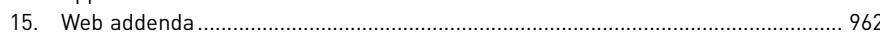

16. References........................ 962

Disclaimer: The ESC/ERS Guidelines represent the views of the ESC and ERS and were produced after careful consideration of the scientific and medical knowledge and the evidence available at the time of their publication. The ESC and ERS are not responsible in the event of any contradiction, discrepancy and/or ambiguity between the ESC/ERS Guidelines and any other official recommendations or guidelines issued by the relevant public health authorities, in particular in relation to good use of healthcare or therapeutic strategies. Health professionals are encouraged to take the ESC/ERS Guidelines fully into account when exercising their clinical judgment, as well as in the determination and the implementation of preventive, diagnostic or therapeutic medical strategies; however, the ESC/ERS Guidelines do not override, in any way whatsoever, the individual responsibility of health professionals to make appropriate and accurate decisions in consideration of each patient's health condition and in consultation with that patient and, where appropriate and/or necessary, the patient's caregiver. Nor do the ESC/ERS Guidelines exempt health professionals from taking into full and careful consideration the relevant official updated recommendations or guidelines issued by the competent public health authorities, in order to manage each patient's case in light of the scientifically accepted data pursuant to their respective ethical and professional obligations. It is also the health professional's responsibility to verify the applicable rules and regulations relating to drugs and medical devices at the time of prescription. 
Abbreviations and acronyms

ALAT
ASAT
APAH
BAS
BMPR2
BNP
BPA
BREATHE
CAV1
CCB
CGMP
CHD
CI
CMR
CO
COPD
CPC-PH
CPET
CPFE
CT
CTD
CTPA
CTEPH
DLCO
DPAH
DPG
EACVI
ECG
ECMO
EIF2AK4
EMA
ERA
FC
FDA
HAART
HIV
HF-pEF
HPAH
HRCT
ICU
INR
IPAH
IPC-PH
IPF

Antagonist THErapy

caveolin-1

cardiac index

cardiac output

hypertension

hypertension

monoxide

electrocardiogram

functional class

ejection fraction

intensive care unit alanine aminotransferase

aspartate aminotransferase

associated pulmonary arterial hypertension

balloon atrial septostomy

bone morphogenetic protein receptor 2

brain natriuretic peptide

balloon pulmonary angioplasty

Bosentan Randomised trial of Endothelin

calcium channel blocker

cyclic guanosine monophosphate

congenital heart disease

cardiac magnetic resonance

chronic obstructive pulmonary disease

combined post-capillary and pre-capillary pulmonary

cardiopulmonary exercise testing

combined pulmonary fibrosis and emphysema

computed tomography

connective tissue disease

computed tomography pulmonary angiogram

chronic thromboembolic pulmonary

diffusing capacity of the lung for carbon

drug-induced pulmonary arterial hypertension

diastolic pressure gradient (diastolic PAP - mean PAWP)

European association of cardiovascular imaging

extracorporeal membrane oxygenation

eukaryotic translation initiation factor 2 alpha kinase 4

European Medicines Agency

endothelin receptor antagonist

US Food and Drug Administration

highly active antiretroviral therapy

human immunodeficiency virus

heart failure with preserved left ventricular

heritable pulmonary arterial hypertension

high-resolution computed tomography

international normalized ratio

idiopathic pulmonary arterial hypertension

isolated post-capillary pulmonary hypertension

idiopathic pulmonary fibrosis

\begin{tabular}{|c|c|}
\hline i.v. & intravenous \\
\hline IVC & inferior vena cava \\
\hline LA & left atrium/atrial \\
\hline LHD & left heart disease \\
\hline LV & left ventricle/ventricular \\
\hline MR & magnetic resonance \\
\hline NYHA & New York Heart Association \\
\hline NO & nitric oxide \\
\hline NT-proBNP & $\mathrm{N}$-terminal pro-brain natriuretic peptide \\
\hline PA & pulmonary artery \\
\hline $\mathrm{PaCO}_{2}$ & arterial carbon dioxide pressure \\
\hline $\mathrm{PaO}_{2}$ & arterial oxygen pressure \\
\hline $\mathrm{PAH}$ & pulmonary arterial hypertension \\
\hline PAP & pulmonary arterial pressure \\
\hline PAPm & mean pulmonary arterial pressure \\
\hline PAPs & systolic pulmonary arterial pressure \\
\hline PAWP & pulmonary artery wedge pressure \\
\hline PASP & pulmonary artery systolic pressure \\
\hline $\mathrm{PCH}$ & pulmonary capillary haemangiomatosis \\
\hline PDE-5i & phosphodiesterase type 5 inhibitor \\
\hline PE & pulmonary embolism \\
\hline PEA & pulmonary endarterectomy \\
\hline PFTs & pulmonary function tests \\
\hline $\mathrm{PH}$ & pulmonary hypertension \\
\hline $\mathrm{PoPH}$ & porto-pulmonary hypertension \\
\hline PPHN & $\begin{array}{l}\text { persistent pulmonary hypertension of the } \\
\text { newborn }\end{array}$ \\
\hline PVOD & pulmonary veno-occlusive disease \\
\hline PVR & pulmonary vascular resistance \\
\hline RA & right atrium \\
\hline RAP & right atrial pressure \\
\hline RCT & randomized controlled trial \\
\hline $\mathrm{RHC}$ & right heart catheterization \\
\hline RV & right ventricle/ventricular \\
\hline 6MWD/6MWT & 6-minute walking distance/6-minute walking test \\
\hline SCD & sickle cell disease \\
\hline sGC & soluble guanylate cyclase \\
\hline $\mathrm{SSc}$ & systemic sclerosis \\
\hline $\mathrm{SvO}_{2}$ & mixed venous oxygen saturation \\
\hline SVR & systemic vascular resistance \\
\hline TAPSE & tricuspid annular plane systolic excursion \\
\hline t.i.d. & three times a day \\
\hline TGF- $\beta$ & transforming growth factor $\beta$ \\
\hline TPG & $\begin{array}{l}\text { transpulmonary pressure gradient (mean PAP - mean } \\
\text { PAWP) }\end{array}$ \\
\hline TRV & tricuspid regurgitant velocity \\
\hline $\mathrm{VE} / \mathrm{VCO}_{2}$ & $\begin{array}{l}\text { minute ventilation - carbon dioxide production } \\
\text { relationship }\end{array}$ \\
\hline$V / Q$ & ventilation/perfusion \\
\hline WHO-FC & World Health Organization functional class \\
\hline WU & Wood units \\
\hline
\end{tabular}

\section{Preamble}

Guidelines summarize and evaluate all available evidence on a particular issue at the time of the writing process, with the aim of assisting health professionals in selecting the best management strategies for an individual patient with a given condition, taking into account the impact on outcome, as well as the riskbenefit ratio of particular diagnostic or therapeutic means. Guidelines and recommendations should help health professionals to make decisions in their daily practice. However, the final decisions concerning an individual patient must be made by the responsible health professional(s) in consultation with the patient and caregiver as appropriate.

A great number of Guidelines have been issued in recent years by the European Society of Cardiology (ESC) and by the European Respiratory Society (ERS), as well as by other societies and organisations. Because of the impact on clinical practice, quality criteria for the development of guidelines have been established in order to make all decisions transparent to the user. The recommendations for formulating and issuing ESC Guidelines can be found on the ESC website (http://www.escardio.org/ Guidelines-\&-Education/Clinical-Practice-Guidelines/Guidelines-development/Writing-ESC-Guidelines). ESC Guidelines represent the official position of the ESC on a given topic and are regularly updated.

Members of this Task Force were selected by the ESC and ERS to represent professionals involved with the medical care of patients with this pathology. Selected experts in the field undertook a comprehensive 
review of the published evidence for management (including diagnosis, treatment, prevention and rehabilitation) of a given condition according to ESC Committee for Practice Guidelines (CPG) policy and approved by the ERS. A critical evaluation of diagnostic and therapeutic procedures was performed, including assessment of the risk-benefit ratio. Estimates of expected health outcomes for larger populations were included, where data exist. The level of evidence and the strength of the recommendation of particular management options were weighed and graded according to predefined scales, as outlined in Tables 1 and 2 .

The experts of the writing and reviewing panels provided declaration of interest forms for all relationships that might be perceived as real or potential sources of conflicts of interest. These forms were compiled into one file and can be found on the ESC website (http://www.escardio.org/guidelines). Any changes in declarations of interest that arise during the writing period must be notified to the ESC and ERS and updated. The Task Force received its entire financial support from the ESC and ERS without any involvement from the healthcare industry.

The ESC CPG supervises and coordinates the preparation of new Guidelines produced by task forces, expert groups or consensus panels. The Committee is also responsible for the endorsement process of these Guidelines. The ESC Guidelines undergo extensive review by the CPG and external experts, and in this case by ERS-appointed experts. After appropriate revisions the Guidelines are approved by all the experts involved in the Task Force. The finalized document is approved by the CPG and by ERS for publication in the European Heart Journal and in the European Respiratory Journal. The Guidelines were developed after careful consideration of the scientific and medical knowledge and the evidence available at the time of their dating.

The task of developing ESC/ERS Guidelines covers not only integration of the most recent research, but also the creation of educational tools and implementation programmes for the recommendations. To implement the guidelines, condensed pocket guideline versions, summary slides, booklets with essential messages, summary cards for non-specialists and an electronic version for digital applications (smartphones, etc.) are produced. These versions are abridged and thus, if needed, one should always refer to the full text version, which is freely available on the ESC website. The National Societies of the ESC are encouraged to endorse, translate and implement all ESC Guidelines. Implementation programmes are needed because it has been shown that the outcome of disease may be favourably influenced by the thorough application of clinical recommendations.

Surveys and registries are needed to verify that real-life daily practice is in keeping with what is recommended in the guidelines, thus completing the loop between clinical research, writing of guidelines, disseminating them and implementing them into clinical practice.

\section{TABLE 1 Classes of recommendations}

\begin{tabular}{|c|c|c|}
\hline $\begin{array}{l}\text { Classes of } \\
\text { recommendations }\end{array}$ & Definition & Suggested wording to use \\
\hline Class I & $\begin{array}{l}\text { Evidence and/or general agreement that a } \\
\text { given treatment or procedure is beneficial, } \\
\text { useful, effective. }\end{array}$ & Is recommended/is indicated \\
\hline Class II & $\begin{array}{l}\text { Conflicting evidence and/or a divergence of } \\
\text { opinion about the usefulness/efficacy of the } \\
\text { given treatment or procedure. }\end{array}$ & \\
\hline Class I/a & $\begin{array}{l}\text { Weight of evidence/opinion is in favour of } \\
\text { usefulness/efficacy. }\end{array}$ & Should be considered \\
\hline Class $/ / b$ & $\begin{array}{l}\text { Usefulness/efficacy is less well established by } \\
\text { evidence/opinion. }\end{array}$ & May be considered \\
\hline Class III & $\begin{array}{l}\text { Evidence or general agreement that the } \\
\text { given treatment or procedure is not } \\
\text { useful/effective, and in some cases may be } \\
\text { harmful. }\end{array}$ & Is not recommended \\
\hline
\end{tabular}


TABLE 2 Level of evidence

\begin{tabular}{|c|c|}
\hline $\begin{array}{c}\text { Level of } \\
\text { evidence A }\end{array}$ & $\begin{array}{c}\text { Data derived from multiple randomized clinical trials } \\
\text { or meta-analyses. }\end{array}$ \\
\hline $\begin{array}{c}\text { Level of } \\
\text { evidence B }\end{array}$ & $\begin{array}{c}\text { Data derived from a single randomized clinical trial or } \\
\text { large non-randomised studies. }\end{array}$ \\
\hline $\begin{array}{c}\text { Level of } \\
\text { evidence C }\end{array}$ & $\begin{array}{c}\text { Consensus of opinion of the experts and/or small } \\
\text { studies, retrospective studies, registries. }\end{array}$ \\
\hline
\end{tabular}

Health professionals are encouraged to take the ESC/ERS Guidelines fully into account when exercising their clinical judgment, as well as in the determination and the implementation of preventive, diagnostic or therapeutic medical strategies. However, the ESC/ERS Guidelines do not override in any way whatsoever the individual responsibility of health professionals to make appropriate and accurate decisions in consideration of each patient's health condition and in consultation with that patient and the patient's caregiver where appropriate and/or necessary. It is also the health professional's responsibility to verify the rules and regulations applicable to drugs and devices at the time of prescription.

\section{Introduction}

Pulmonary hypertension $(\mathrm{PH})$ is a pathophysiological disorder that may involve multiple clinical conditions and can complicate the majority of cardiovascular and respiratory diseases. The composition of the guidelines task force reflects the multidisciplinary nature of $\mathrm{PH}$, including members of different medical societies, associations and working groups. The current document follows the two previous ESC and ERS Guidelines, published in 2004 and 2009, focusing on clinical management of PH. A systematic literature review was performed from MEDLINE $^{\oplus}$ to identify new studies published since 2009 concerning the topic of PH. Task force members selected studies based on relevance and appropriateness. The main changes and adaptations as compared with the 2009 ESC and ERS PH guidelines are as follows:

- The table of contents structure has been simplified, with three initial general chapters including classifications, basic aspects and differential diagnosis, two chapters for pulmonary arterial hypertension (PAH) and one chapter each for PH due to left heart disease (LHD), lung disease and/or hypoxia, chronic thromboembolic pulmonary hypertension $(\mathrm{CTEPH})$ and unclear and/or multifactorial mechanisms.

- New wordings and parameters for the haemodynamic definition of post-capillary PH subgroups have been adopted. Pulmonary vascular resistance (PVR) has been included in the haemodynamic definition of PAH.

- An updated common clinical classification for adult and paediatric patients is reported.

- New advances in pathology, pathobiology, genetics, epidemiology and risk factors are reported.

- An updated diagnostic algorithm has been provided in an independent chapter and novel screening strategies are proposed in the web addenda.

- The importance of expert referral centres in the management of PH patients has been highlighted in both the diagnostic and treatment algorithms.

- New developments on PAH severity evaluation and on treatments and treatment goals are reported, including combination therapy and two new recently approved drugs. The treatment algorithm has been updated accordingly.

- The chapters on PH due to LHD and lung diseases have been updated. The term 'out of proportion PH' has been abandoned in both conditions.

- New diagnostic and treatment algorithms are reported in the CTEPH chapter, including general criteria for operability and balloon pulmonary angioplasty (BPA) and a newly approved drug.

- A short chapter on PH due to unclear and/or multifactorial mechanisms has been added.

\section{Definitions and classifications}

\subsection{Definitions}

$\mathrm{PH}$ is defined as an increase in mean pulmonary arterial pressure (PAPm) $\geqslant 25 \mathrm{mmHg}$ at rest as assessed by right heart catheterization (RHC) [1]. Available data have shown that the normal PAPm at rest is $14 \pm 3 \mathrm{mmHg}$ with an upper limit of normal of approximately $20 \mathrm{mmHg}[1,2]$. The clinical significance of a PAPm between 21 and $24 \mathrm{mmHg}$ is unclear. Patients presenting with a pulmonary artery pressure (PAP) in this range should be carefully followed when they are at risk for developing PAH [e.g. patients with connective tissue disease (CTD) or family members of patients with heritable PAH (HPAH)] [1]. 
Due to the lack of reliable data that define which levels of exercise-induced changes in PAPm or PVR have prognostic implications, a disease entity 'PH on exercise' cannot be defined and should not be used [1]. A recent retrospective study has proposed a definition of $\mathrm{PH}$ on exercise with the combination of PAPm and total PVR data, but no outcome prospective validation has been provided [3].

The term $\mathrm{PAH}$ describes a group of $\mathrm{PH}$ patients characterized haemodynamically by the presence of pre-capillary $\mathrm{PH}$, defined by a pulmonary artery wedge pressure (PAWP) $\leqslant 15 \mathrm{mmHg}$ and a PVR $>3$ Wood units (WU) in the absence of other causes of pre-capillary $\mathrm{PH}$ such as $\mathrm{PH}$ due to lung diseases, CTEPH or other rare diseases [1].

According to various combinations of PAP, PAWP, cardiac output (CO), diastolic pressure gradient (DPG) and PVR, assessed in stable clinical conditions, different haemodynamic definitions of $\mathrm{PH}$ are shown in Table 3 together with their corresponding clinical classification (Table 4) $[1,4]$. The reasons for the updated definitions of post-capillary $\mathrm{PH}$ are reported in the specific section (8.0).

\subsection{Classifications}

The clinical classification of $\mathrm{PH}$ is intended to categorize multiple clinical conditions into five groups according to their similar clinical presentation, pathological findings, haemodynamic characteristics and treatment strategy [5]. The clinical classification may be updated when new data are available on the above features or when additional clinical entities are considered. A comprehensive version of the clinical classification is presented in Table 4 [6]. A condensed version is provided in a web addenda (Web Table I).

The new findings are as follows:

- New conditions that are frequently found in children have been included in different clinical groups in order to provide a comprehensive classification appropriate to both adult and paediatric patients.

- Recently identified gene mutations have been included in the HPAH subgroup of clinical group 1 (PAH). The new mutations are more rare as compared with the traditional bone morphogenetic protein receptor 2 (BMPR2) mutations (Table 4).

- Pre-capillary PH associated with chronic haemolytic anaemia appears to be significantly different from other forms of PAH in regard to pathological findings (absence of plexiform lesions), haemodynamic characteristics (low PVR and high CO) and response to PAH-specific therapies (no demonstration of efficacy). Therefore these clinical conditions have been moved from group 1 (PAH) to group 5 (unclear and/or multifactorial mechanisms).

- Group $1^{\prime}$ [pulmonary veno-occlusive disease (PVOD) and/or pulmonary capillary haemangiomatosis $(\mathrm{PCH})]$ has been expanded and includes idiopathic, heritable, drug-, toxin- and radiation-induced and associated forms.

- Persistent PH of the newborn (PPHN) includes a heterogeneous group of conditions that may differ from classical PAH. As a consequence, PPHN has been subcategorised as group 1" [7-9].

\section{TABLE 3 Haemodynamic definitions of pulmonary hypertension ${ }^{a}$}

\begin{tabular}{|c|c|c|}
\hline Definition & Characteristics $^{\circ}$ & Clinical group (s)b \\
\hline $\mathrm{PH}$ & $\mathrm{PAPm} \geqslant 25 \mathrm{mmHg}$ & All \\
\hline Pre-capillary PH & $\begin{array}{l}\text { PAPm } \geqslant 25 \mathrm{mmHg} \\
\text { PAWP } \leqslant 15 \mathrm{mmHg}\end{array}$ & $\begin{array}{l}\text { 1. Pulmonary arterial hypertension } \\
\text { 3. PH due to lung diseases } \\
\text { 4. Chronic thromboembolic } \mathrm{PH} \\
\text { 5. PH with unclear and/or } \\
\text { multifactorial mechanisms }\end{array}$ \\
\hline Post-capillary PH & $\begin{array}{l}\text { PAPm } \geqslant 25 \mathrm{mmHg} \\
\text { PAWP }>15 \mathrm{mmHg}\end{array}$ & $\begin{array}{l}\text { 2. } \mathrm{PH} \text { due to left heart disease } \\
\text { 5. } \mathrm{PH} \text { with unclear and/or } \\
\text { multifactorial mechanisms }\end{array}$ \\
\hline $\begin{array}{l}\text { Isolated post-capillary PH } \\
\text { (Ipc-PH) }\end{array}$ & $\begin{array}{l}\mathrm{DPG}<7 \mathrm{mmHg} \text { and/or } \\
\mathrm{PVR} \leqslant 3 \mathrm{WUc}\end{array}$ & \\
\hline $\begin{array}{l}\text { Combined post-capillary and pre-capillary } \mathrm{PH} \\
(\mathrm{Cpc}-\mathrm{PH})\end{array}$ & $\begin{array}{l}\mathrm{DPG} \geqslant 7 \mathrm{mmHg} \text { and/or } \\
\mathrm{PVR}>3 \mathrm{WUc}\end{array}$ & \\
\hline
\end{tabular}

CO: cardiac output; DPG: diastolic pressure gradient (diastolic PAP - mean PAWP); mPAP: mean pulmonary arterial pressure; PAWP: pulmonary arterial wedge pressure; $\mathrm{PH}$ : pulmonary hypertension;

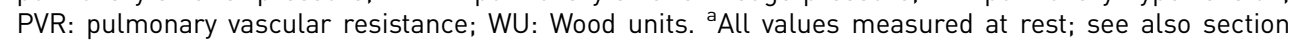
8.0. ${ }^{b}$ According to Table 4. ' Wood Units are preferred to dynes.s. $\mathrm{cm}^{-5}$. 
- Paediatric heart diseases such as congenital or acquired left heart inflow or outflow tract obstruction and congenital cardiomyopathies have been included in group 2 ( $\mathrm{PH}$ due to LHD).

- No changes are proposed for group 3 (PH due to lung diseases and/or hypoxia).

- Group 4 has been renamed as 'CTEPH and other pulmonary artery (PA) obstructions', which includes $\mathrm{CTEPH}$, pulmonary angiosarcoma, other intravascular tumours, arteritis, congenital pulmonary arteries stenoses and parasites (Table 4).

- Segmental PH is observed in discrete lung areas perfused by aorto-pulmonary collaterals in congenital heart diseases such as pulmonary or tricuspid atresia. This very unusual haemodynamic condition has been included in group 5 (unclear and/or multifactorial mechanisms).

- Some pathological and pathophysiological information on the clinical groups are reported in the web addenda.

Important pathophysiological and clinical definitions are reported in Table 5. A clinical classification of PAH associated with congenital heart disease (CHD) is reported in Table 6.

An anatomical-pathophysiological classification of congenital systemic-to-pulmonary shunts associated with PAH is presented in Web Table II. A list of developmental lung diseases associated with $\mathrm{PH}$ is presented in Web Table III.

\section{Epidemiology and genetics of pulmonary hypertension \\ 4.1 Epidemiology and risk factors}

Reporting in the literature of $\mathrm{PH}$ incidence data at the global level is poor. In the UK, a prevalence of 97 cases per million with a female:male ratio of 1.8 has been reported. The age-standardized death rate in the USA ranges between 4.5 and 12.3 per 100,000 population. Comparative epidemiological data on the prevalence of the different groups of $\mathrm{PH}$ are not widely available, but it is clear that LHD (group 2) is believed to be the most common cause of $\mathrm{PH}$, although severe $\mathrm{PH}$ is relatively uncommon in this setting. Although patients belonging to groups 2 and 3 represent an important part of the clinical practice, there is disproportionately little information about the demographics and clinical course of this segment of the $\mathrm{PH}$ population, suggesting that registry database methodology may be useful for these groups. Globally, schistosomiasis-associated PAH and high altitude-related $\mathrm{PH}$ represent an important burden to mankind.

- Group 1 (PAH): Several registries have described the epidemiology of PAH [10-12]. The lowest estimate of the prevalence of PAH and idiopathic PAH (IPAH) are 15 cases and 5.9 cases per million adult population, respectively. The lowest estimate of $\mathrm{PAH}$ incidence is 2.4 cases per million adult population per year. In Europe, PAH prevalence and incidence are in the range of 15-60 subjects per million population and 5-10 cases per million per year, respectively [11]. In registries, around half of PAH patients have idiopathic, heritable or drug-induced PAH. In the subgroup of associated PAH conditions (APAH), the leading cause is CTD, mainly systemic sclerosis (SSc) [10].

PAH may occur in different settings depending on associated clinical conditions [13]. IPAH corresponds to sporadic disease, without any familial history of PAH or known triggering factor. While the mean age of patients with IPAH in the first US National Institutes of Health registry created in 1981 was 36 years, PAH is now more frequently diagnosed in elderly patients, resulting in a mean age at diagnosis between 50 and 65 years in current registries. Furthermore, the female predominance is quite variable among registries and may not be present in elderly patients, and survival appears to have improved over time.

A number of risk factors for the development of PAH has been identified and are defined as any factor or condition that is suspected to play a predisposing or facilitating role in disease development. Risk factors were classified as definite, likely or possible, based on the strength of their association with $\mathrm{PH}$ and their probable causal role [13]. A definite association is acknowledged in the case of either an epidemic, such as occurred with appetite suppressants, or if large, multicentre epidemiological studies demonstrate an association between the clinical condition or drug and PAH. A likely association is acknowledged if a single-centre case-control study or multiple case series demonstrate an association or if clinical and haemodynamic recovery occurs after stopping exposure, such as occurred in dasatinib-induced PAH. A possible association can be suspected, for example, for drugs with similar mechanisms of action as those in the definite or likely category but which have not yet been studied, such as drugs used to treat attention deficit disorder. Definite clinical associations are listed among APAH in Table 4 and the risk level of different drugs and toxins are listed in Table 7 [6, 14-16].

- Group 2 (PH due to LHD): The prevalence of $\mathrm{PH}$ in patients with chronic heart failure increases with the progression of functional class (FC) impairment. Up to $60 \%$ of patients with severe left ventricular (LV) systolic dysfunction and up to $70 \%$ of patients with heart failure with preserved ejection fraction may present with $\mathrm{PH}$. In left-sided valvular diseases, the prevalence of $\mathrm{PH}$ increases with the severity of the defect and of the symptoms. $\mathrm{PH}$ can be found in virtually all patients with severe symptomatic mitral valve disease and in up to $65 \%$ of those with symptomatic aortic stenosis [17-19]. 
TABLE 4 Comprehensive clinical classification of pulmonary hypertension (updated from Simonneau et al. [5])

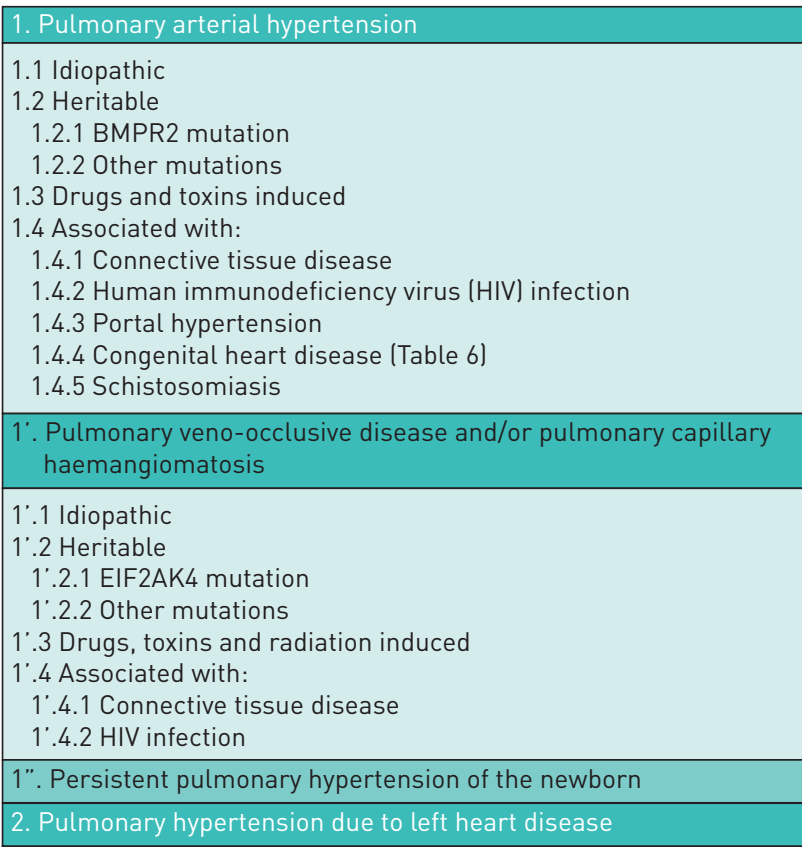

2.1 Left ventricular systolic dysfunction

2.2 Left ventricular diastolic dysfunction

2.3 Valvular disease

2.4 Congenital/acquired left heart inflow/outflow tract obstruction and congenital cardiomyopathies

2.5 Congenital/acquired pulmonary veins stenosis

3. Pulmonary hypertension due to lung diseases and/or hypoxia

3.1 Chronic obstructive pulmonary disease

3.2 Interstitial lung disease

3.3 Other pulmonary diseases with mixed restrictive and obstructive pattern

3.4 Sleep-disordered breathing

3.5 Alveolar hypoventilation disorders

3.6 Chronic exposure to high altitude

3.7 Developmental lung diseases (Web Table III)

4. Chronic thromboembolic pulmonary hypertension and other pulmonary artery obstructions

4.1 Chronic thromboembolic pulmonary hypertension

4.2 Other pulmonary artery obstructions

4.2.1 Angiosarcoma

4.2.2 Other intravascular tumors

4.2.3 Arteritis

4.2.4 Congenital pulmonary arteries stenoses

4.2.5 Parasites (hydatidosis)

5. Pulmonary hypertension with unclear and/or multifactorial mechanisms

5.1 Haematological disorders: chronic haemolytic anaemia, myeloproliferative disorders, splenectomy

5.2 Systemic disorders: sarcoidosis, pulmonary histiocytosis, lymphangioleiomyomatosis, neurofibromatosis

5.3 Metabolic disorders: glycogen storage disease, Gaucher disease, thyroid disorders

5.4 Others: pulmonary tumoral thrombotic microangiopathy, fibrosing mediastinitis, chronic renal failure (with/without dialysis), segmental pulmonary hypertension

BMPR2: bone morphogenetic protein receptor, type 2 EIF2AK4: eukaryotic translation initiation factor 2 alpha kinase 4; HIV: human immunodeficiency virus.
TABLE 5 Important pathophysiological and clinical definitions

1. Pulmonary hypertension $(\mathrm{PH})$ is a haemodynamic and pathophysiological condition defined as an increase in mean pulmonary arterial pressure $\geqslant 25 \mathrm{mmHg}$ at rest as assessed by right heart catheterization (Table 3). PH can be found in multiple clinical conditions (Table 4).

2. Pulmonary arterial hypertension (PAH, group 1) is a clinical condition characterized by the presence of pre-capillary PH (Table 3) and pulmonary vascular resistance $>3$ Wood units, in the absence of other causes of pre-capillary $\mathrm{PH}$ such as $\mathrm{PH}$ due to lung diseases, chronic thromboembolic $\mathrm{PH}$, or other rare diseases (Table 4). PAH includes different forms that share a similar clinical picture and virtually identical pathological changes of the lung microcirculation (Table 4).

3. There is no sufficient data to support the definition of ' $\mathrm{PH}$ on exercise'.

\section{TABLE 6 Clinical classification of pulmonary arterial hypertension associated with congenital heart disease (updated from Simonneau et al. [5]]}

\section{Eisenmenger's syndrome}

Includes all large intra- and extra-cardiac defects which begin as systemic-to-pulmonary shunts and progress with time to severe elevation of PVR and to reversal (pulmonary-to-systemic) or bidirectional shunting; cyanosis, secondary erythrocytosis, and multiple organ involvement are usually present.

2. PAH associated with prevalent systemic-to-pulmonary shunts

- Correctablea

- Non-correctable

Includes moderate to large defects; PVR is mildly to moderately increased, systemic-to-pulmonary shunting is still prevalent, whereas cyanosis at rest is not a feature.

3. PAH with small/coincidental defects ${ }^{b}$

Marked elevation in PVR in the presence of small cardiac defects lusually ventricular septal defects $<1 \mathrm{~cm}$ and atrial septal defects $<2 \mathrm{~cm}$ of effective diameter assessed by echol, which themselves do not account for the development of elevated PVR; the clinical picture is very similar to idiopathic PAH. Closing the defects is contra-indicated.

4. PAH after defect correction

Congenital heart disease is repaired, but PAH either persists immediately after correction or recurs/develops months or years after correction in the absence of significant postoperative haemodynamic lesions.

PAH: pulmonary arterial hypertension; PVR: pulmonary vascular resistance. ${ }^{a}$ With surgery or intravascular percutaneous procedure. ${ }^{\mathrm{b}}$ The size applies to adult patients. However, also in adults the simple diameter may be not sufficient for defining the haemodynamic relevance of the defect and also the pressure gradient, the shunt size and direction, and the pulmonary to systemic flows ratio should be considered (Web Table II). 
TABLE 7 Updated risk level of drugs and toxins known to induce pulmonary arterial hypertension

\begin{tabular}{|l|l|l|}
\hline \multicolumn{1}{|c|}{ Definite } & \multicolumn{1}{|c|}{ Likely } & \multicolumn{1}{c|}{ Possible } \\
\hline - Aminorex & - Amphetamines & - Cocaine \\
- Fenfluramine & - Dasatinib & - Phenylpropanolamine \\
- Dexfenfluramine & - L-tryptophan & - St John's Wort \\
$\begin{array}{l}\text { - Toxic rapeseed oil } \\
\text { - Senfluorex } \\
\text { reuptake inhibitors }\end{array}$ & - Methamphetamines & - Amphetamine-like drugs \\
\hline
\end{tabular}

ancreased risk of persistent pulmonary hypertension in the newborns of mothers with intake of selective serotonin reuptake inhibitors; ${ }^{b}$ Alkylating agents are possible causes of pulmonary veno-occlusive disease.

- Group 3 (PH due to lung diseases and/or hypoxaemia): Mild $\mathrm{PH}$ is common in both severe interstitial lung disease and severe chronic obstructive pulmonary disease (COPD) [20], while severe PH is uncommon [21]. Severe PH can be seen in the combined emphysema/fibrosis syndrome, where the prevalence of PH is high [22].

- Group 4 [CTEPH and other PA obstructions]: In the Spanish PH Registry, CTEPH prevalence and incidence were 3.2 cases per million and 0.9 cases per million per year, respectively [23]. Even though a prevalence of CTEPH of $3.8 \%$ has been reported in survivors of acute pulmonary embolism (PE), the true incidence of CTEPH after acute PE is lower, in the range of $0.5-2 \%$ [24]. A history of acute $\mathrm{PE}$ was reported for $74.8 \%$ of patients from the International CTEPH Registry [25]. Associated conditions included thrombophilic disorders (lupus anticoagulant/antiphospholipid antibodies, protein $\mathrm{S}$ and $\mathrm{C}$ deficiency, activated protein $\mathrm{C}$ resistance including factor $\mathrm{V}$ Leiden mutation, prothrombin gene mutation, antithrombin III deficiency and elevated factor VIII) in $31.9 \%$ of patients and splenectomy in $3.4 \%$.

\subsection{Genetics}

- Group 1 (PAH): Heterozygous BMPR2 mutations account for approximately $75 \%$ of familial PAH and up to $25 \%$ of apparently sporadic PAH cases [26]. BMPR2 encodes a type 2 receptor for bone morphogenetic proteins involved in the control of vascular cell proliferation. Mutations of genes coding for activin receptor-like kinase 1 and endoglin have been identified in PAH patients with a personal or family history of hereditary haemorrhagic telangiectasia, as well as in BMPR1B and SMAD9, supporting a prominent role for transforming growth factor $\beta$ (TGF- $\beta$ ) family members in PAH [26]. Whole exome sequencing has identified rare heterozygous mutations in genes coding for proteins such as caveolin 1 (CAV1) and the potassium channel subfamily $\mathrm{K}$ member 3 (KCNK3) [26, 27].

- Group 1: Heritable PVOD/PCH has been recognized in consanguineous families, suggesting recessive transmission. Whole genome sequencing demonstrated that bi-allelic mutations in eukaryotic translation initiation factor 2 alpha kinase 4 (EIF2AK4) were present in all familial PVOD/PCH and in 25\% of histologically confirmed sporadic PVOD/PCH [28]. EIF2AK4 encodes a serine-threonine kinase present in all eukaryotes that can induce changes in gene expression in response to amino acid deprivation.

- Group 2 (PH due to LHD): No specific genetic linkage has been identified [18].

- Group 3 (PH due to lung diseases and/or hypoxaemia): Gene polymorphism might contribute towards determining the severity of PH in hypoxaemic patients with COPD [29].

- Group 4 (CTEPH and other PA obstructions): No specific genetic mutations have been linked to the development of CTEPH.

- Group 5 (PH with unclear and/or multifactorial mechanisms): The heterogeneity of this group prevents an appropriate description of genetics, epidemiology and risk factors in these guidelines.

\section{Pulmonary hypertension diagnosis}

\subsection{Diagnosis}

The diagnosis of $\mathrm{PH}$ requires a clinical suspicion based on symptoms and physical examination and review of a comprehensive set of investigations to confirm that haemodynamic criteria are met and to describe the aetiology and the functional and haemodynamic severity of the condition. The interpretation of these investigations requires, at the very least, expertise in cardiology, imaging and 
respiratory medicine and may best be discussed at a multidisciplinary team meeting. This is particularly important for identifying patients who may have more than one cause of $\mathrm{PH}$. The main cause of $\mathrm{PH}$ should be identified according to the clinical classification in Table 4. An algorithm for reaching a diagnosis is shown in Figure 1.

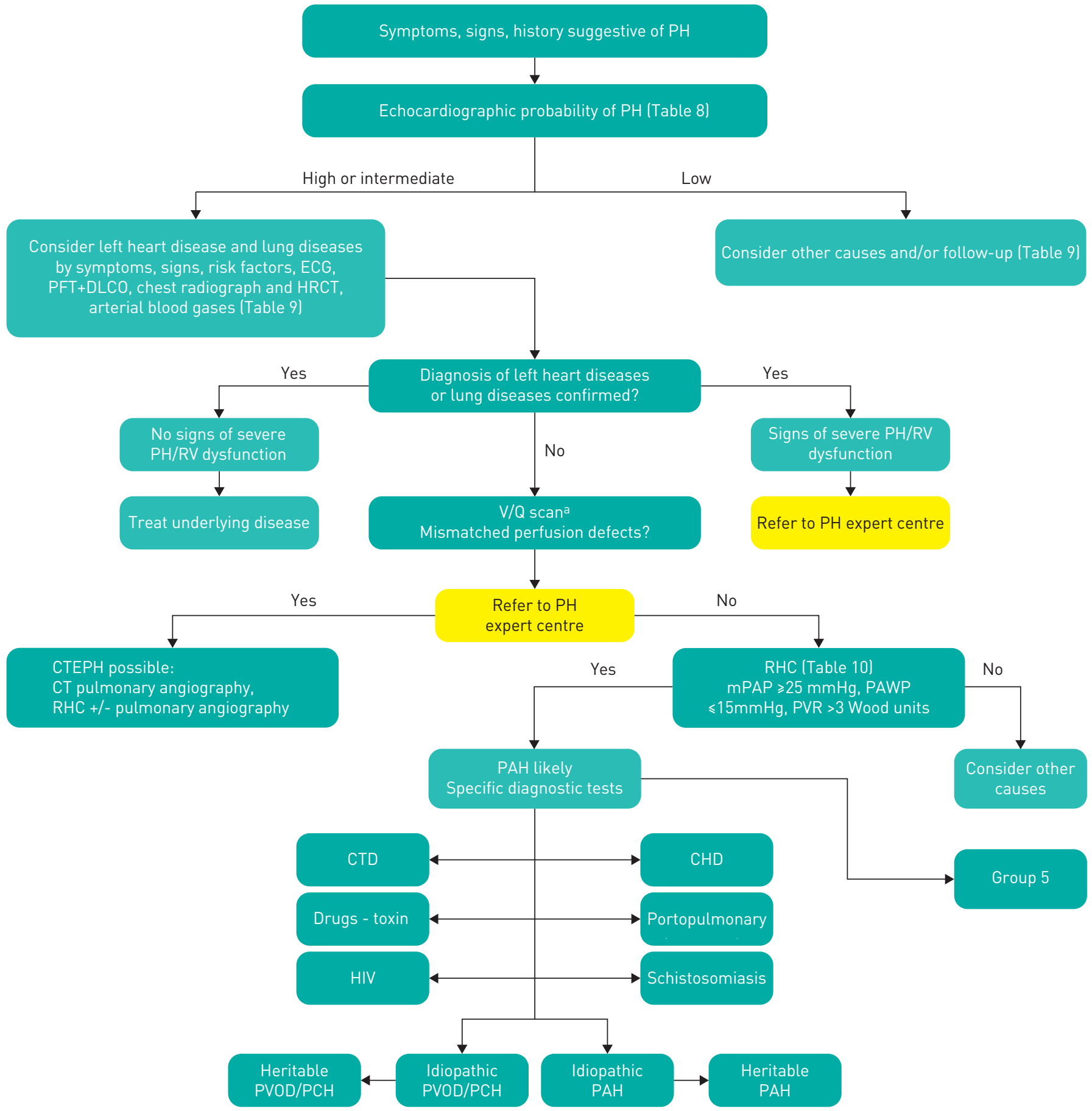

FIGURE 1 Diagnostic algorithm. CHD: congenital heart diseases; CT: computed tomography; CTD: connective tissue disease; CTEPH: chronic thromboembolic pulmonary hypertension; DLCO: carbon monoxide diffusing capacity; ECG: electrocardiogram; HIV: Human immunodeficiency virus; HRCT: high-resolution CT; mPAP: mean pulmonary arterial pressure; PA: pulmonary angiography; PAH: pulmonary arterial hypertension; PAWP: pulmonary artery wedge pressure; PFT: pulmonary function tests; PH: pulmonary hypertension; PVOD/PCH: pulmonary veno-occlusive disease or pulmonary capillary hemangiomathosis; PVR: pulmonary vascular resistance; RHC: right heart catheterisation; RV: right ventricular; $\mathrm{V} / \mathrm{Q}$ : ventilation/perfusion. ${ }^{\mathrm{a}} \mathrm{CT}$ pulmonary angiography alone may miss diagnosis of chronic thromboembolic pulmonary hypertension. 


\subsubsection{Clinical presentation}

The symptoms of $\mathrm{PH}$ are non-specific and mainly related to progressive right ventricular (RV) dysfunction. Initial symptoms are typically induced by exertion. They include shortness of breath, fatigue, weakness, angina and syncope. Less commonly patients may also describe dry cough and exercise-induced nausea and vomiting. Symptoms at rest occur only in advanced cases. Abdominal distension and ankle oedema will develop with progressing RV failure. The presentation of $\mathrm{PH}$ may be modified by diseases that cause or are associated with $\mathrm{PH}$ as well as other concurrent diseases.

In some patients the clinical presentation may be related to mechanical complications of $\mathrm{PH}$ and the abnormal distribution of blood flow in the pulmonary vascular bed. These include haemoptysis related to rupture of hypertrophied bronchial arteries, as well as symptoms attributable to pulmonary arterial dilatation such as hoarseness caused by compression of the left recurrent laryngeal nerve, wheeze caused by large airway compression and angina due to myocardial ischaemia caused by compression of the left main coronary artery. Significant dilation of the PA may result in its rupture or dissection, leading to signs and symptoms of cardiac tamponade.

The physical signs of $\mathrm{PH}$ include left parasternal lift, an accentuated pulmonary component of the second heart sound, an RV third heart sound, a pansystolic murmur of tricuspid regurgitation and a diastolic murmur of pulmonary regurgitation. Elevated jugular venous pressure, hepatomegaly, ascites, peripheral oedema and cool extremities characterize patients with advanced disease. Wheeze and crackles are usually absent.

Clinical examination may suggest an underlying cause of $\mathrm{PH}$. Telangiectasia, digital ulceration and sclerodactyly are seen in scleroderma, inspiratory crackles may point towards interstitial lung disease and spider naevi, testicular atrophy, and palmar erythema suggest liver disease. When digital clubbing is encountered, PVOD, cyanotic CHD, interstitial lung disease or liver disease should be considered.

\subsubsection{Electrocardiogram}

An electrocardiogram (ECG) may provide supportive evidence of $\mathrm{PH}$, but a normal ECG does not exclude the diagnosis. An abnormal ECG is more likely in severe rather than mild PH. ECG abnormalities may include P pulmonale, right axis deviation, RV hypertrophy, RV strain, right bundle branch block, and QTc prolongation. While RV hypertrophy has insufficient sensitivity (55\%) and specificity (70\%) to be a screening tool, RV strain is more sensitive [30]. Prolongation of the QRS complex and QTc suggest severe disease $[31,32]$. The ECG differential diagnosis includes anterolateral myocardial ischaemia. In contrast to $\mathrm{PH}, \mathrm{ECG}$ changes in ischaemia more commonly affect the lateral and inferior leads, and when present in the anterior chest leads are usually accompanied by a Q wave in V1 to V3, and rarely cause right axis deviation.

Supraventricular arrhythmias may occur in advanced disease, in particular atrial flutter, but also atrial fibrillation, with a cumulative incidence in $25 \%$ of patients after 5 years [33]. Atrial arrhythmias compromise $\mathrm{CO}$ and almost invariably lead to further clinical deterioration. Ventricular arrhythmias are rare.

\subsubsection{Chest radiograph}

In $90 \%$ of patients with IPAH the chest radiograph is abnormal at the time of diagnosis [34]. Findings in patients with PAH include central pulmonary arterial dilatation, which contrasts with 'pruning' (loss) of the peripheral blood vessels. Right atrium (RA) and RV enlargement may be seen in more advanced cases. A chest radiograph may assist in differential diagnosis of $\mathrm{PH}$ by showing signs suggesting lung disease (group 3, Table 4) or pulmonary venous congestion due to LHD (group 2, Table 4). Chest radiography may help in distinguishing between arterial and venous $\mathrm{PH}$ by respectively demonstrating increased and decreased artery:vein ratios [35].

Overall, the degree of $\mathrm{PH}$ in any given patient does not correlate with the extent of radiographic abnormalities. As for ECG, a normal chest radiograph does not exclude PH.

\subsubsection{Pulmonary function tests and arterial blood gases}

Pulmonary function tests and arterial blood gases identify the contribution of underlying airway or parenchymal lung disease. Patients with PAH have usually mild to moderate reduction of lung volumes related to disease severity [36, 37]. Although diffusion capacity can be normal in PAH, most patients have decreased lung diffusion capacity for carbon monoxide (DLCO). An abnormal low DLCO, defined as $<45 \%$ of predicted, is associated with a poor outcome $[36,37]$. The differential diagnosis of a low DLCO in PAH includes PVOD, PAH associated with scleroderma and parenchymal lung disease. Although airflow obstruction is unusual, peripheral airway obstruction can be detected. Due to alveolar hyperventilation at rest, arterial oxygen pressure $\left(\mathrm{PaO}_{2}\right)$ remains normal or is only slightly lower than normal and arterial carbon dioxide pressure $\left(\mathrm{PaCO}_{2}\right)$ is decreased [38].

COPD as a cause of hypoxic PH is diagnosed on the evidence of irreversible airflow obstruction together with increased residual volumes and reduced DLCO [39]. Arterial blood gases of COPD patients show a decreased 
$\mathrm{PaO}_{2}$ with normal or increased $\mathrm{PaCO}_{2}$ [40]. A decrease in lung volume combined with decreased diffusion capacity for carbon monoxide may indicate interstitial lung disease [39]. The severity of emphysema and of interstitial lung disease can be diagnosed using high-resolution computed tomography (CT). Combined emphysema and pulmonary fibrosis may pseudonormalize spirometry, although the DLCO is almost always reduced, emphasizing the need to interpret pulmonary function alongside lung imaging.

The prevalence of nocturnal hypoxaemia and central sleep apnoeas are high in PAH (70-80\%) [41, 42]. Overnight oximetry or polysomnography should be performed where obstructive sleep apnoea syndrome or hypoventilation are considered.

\subsubsection{Echocardiography}

Transthoracic echocardiography is used to image the effects of $\mathrm{PH}$ on the heart and estimate PAP from continuous wave Doppler measurements. Echocardiography should always be performed when $\mathrm{PH}$ is suspected and may be used to infer a diagnosis of $\mathrm{PH}$ in patients in whom multiple different echocardiographic measurements are consistent with this diagnosis. When treatment of $\mathrm{PH}$ itself is being considered, echocardiography alone is not sufficient to support a treatment decision and cardiac catheterization is required. Detailed guidelines describing the echocardiographic assessment of the right heart can be found in documents created and/or endorsed by the European Association of Cardiovascular Imaging (EACVI), a registered branch of the ESC, and the reader is referred to these for further instruction [43, 44].

The estimation of systolic PAP is based on the peak tricuspid regurgitation velocity (TRV) taking into account right atrial pressure (RAP) as described by the simplified Bernoulli equation. RAP can be estimated by echocardiography based on the diameter and respiratory variation in diameter of the inferior vena cava (IVC): an IVC diameter $<2.1 \mathrm{~cm}$ that collapses $>50 \%$ with a sniff suggests a normal RA pressure of 3 $\mathrm{mmHg}$ (range $0-5 \mathrm{mmHg}$ ), whereas an IVC diameter $>2.1 \mathrm{~cm}$ that collapses $<50 \%$ with a sniff or $<20 \%$ on quiet inspiration suggests a high RA pressure of $15 \mathrm{mmHg}$ (range 10-20 $\mathrm{mmHg}$ ). In scenarios in which the IVC diameter and collapse do not fit this paradigm, an intermediate value of $8 \mathrm{mmHg}$ (range $5-10 \mathrm{mmHg}$ ) may be used. The EACVI recommends such an approach rather than using a fixed value of 5 or $10 \mathrm{mmHg}$ for PA systolic pressure (PASP) estimations. However, given the inaccuracies of RAP estimation and the amplification of measurement errors by using derived variables, we recommend using the continuous wave Doppler measurement of peak TRV (and not the estimated PASP) as the main variable for assigning the echocardiographic probability of $\mathrm{PH}$.

When peak TRV is technically difficult to measure (trivial or mild tricuspid regurgitation) some laboratories use contrast echocardiography [e.g. agitated saline administered by intravenous (i.v.) injection], which may improve the Doppler signal, allowing measurement of peak TRV velocity. Unfortunately, despite the strong correlation of TRV with a tricuspid regurgitation pressure gradient, Doppler-derived pressure estimation may be inaccurate in the individual patient. In patients with severe tricuspid regurgitation, TRV may be significantly underestimated and cannot be used to exclude $\mathrm{PH}$. Overestimation may also occur [44]. PH cannot be reliably defined by a cut-off value of TRV. Consequently, estimation of PAP based solely on Doppler transthoracic echocardiography measurements is not suitable for screening for mild, asymptomatic PH. Other echocardiographic variables that might raise or reinforce suspicion of PH independent of TRV should always be sought.

Conclusions derived from an echocardiographic examination should aim to assign a level of probability of $\mathrm{PH}$. This ESC Guideline suggests grading the probability of $\mathrm{PH}$ based on TRV at rest and on the presence of additional pre-specified echocardiographic variables suggestive of $\mathrm{PH}$ (Table $8 A$ ). The probability of $\mathrm{PH}$ may then be judged as high, intermediate or low. When interpreted in a clinical context, the echocardiographic result is required to decide the need for cardiac catheterization in individual patients. In order to facilitate and standardize assignment to the level of probability of $\mathrm{PH}$, several additional echocardiographic signs are proposed in addition to criteria based on TRV (Table $8 B$ ). These signs provide assessment of the RV size and pressure overload, the pattern of blood flow velocity out of the RV, the diameter of the PA and an estimate of RAP [43-45]. Their measurement has been defined in recommendations endorsed by the EACVI $[43,44]$.

The recommended plan for further patient investigation based on echocardiographic probability of $\mathrm{PH}$ is shown in Table 9 for symptomatic patients. In the Web addendum, a similar table (Web Table IX) for screening for asymptomatic patients with risk factors for PAH or with incidental findings suggesting the possibility of PH on ECG or lung imaging is provided.

Echocardiography can be helpful in detecting the cause of suspected or confirmed PH. Two-dimensional, Doppler and contrast examinations can be used to identify CHD. High pulmonary blood flow found on pulsed wave Doppler in the absence of a detectable shunt or significant dilatation of proximal PA despite only moderate $\mathrm{PH}$ may warrant transoesophageal examination with contrast or cardiac magnetic 
TABLE 8A Echocardiographic probability of pulmonary hypertension in symptomatic patients with a suspicion of pulmonary hypertension

\begin{tabular}{|c|c|c|}
\hline $\begin{array}{c}\text { Peak tricuspid } \\
\text { regurgitation } \\
\text { velocity }(\mathrm{m} / \mathrm{s})\end{array}$ & $\begin{array}{c}\text { Presence of other } \\
\text { echo 'PH signs'a }\end{array}$ & $\begin{array}{c}\text { Echocardiographic } \\
\text { probability of pulmonary } \\
\text { hypertension }\end{array}$ \\
\hline $\begin{array}{c}\leqslant 2.8 \text { or not } \\
\text { measurable }\end{array}$ & No & Low \\
\hline \begin{tabular}{c|c|}
$\leqslant 2.8$ or not \\
measurable
\end{tabular} & Yes & Intermediate \\
\hline $2.9-3.4$ & No & High \\
\hline $2.9-3.4$ & Yes & Not required \\
\hline$>3.4$ & & \\
\hline
\end{tabular}

$\mathrm{PH}:$ pulmonary hypertension. ${ }^{\mathrm{a} S e e}$ Table $8 B$.
TABLE 8B Echocardiographic signs suggesting pulmonary hypertension used to assess the probability of pulmonary hypertension in addition to tricuspid regurgitation velocity measurement in Table $8 \mathrm{~A}$

\begin{tabular}{|l|l|l|}
\hline A: The ventricles & B: Pulmonary artery & $\begin{array}{l}\text { C: Inferior vena cava and } \\
\text { right atriuma }\end{array}$ \\
\hline $\begin{array}{l}\text { Right ventricle/left } \\
\text { ventricle basal } \\
\text { diameter ratio } \\
>1.0\end{array}$ & $\begin{array}{l}\text { Right ventricular } \\
\text { outflow Doppler } \\
\text { acceleration time } \\
<105 \mathrm{msec} \text { and/or } \\
\text { midsystolic notching }\end{array}$ & $\begin{array}{l}\text { Inferior cava diameter } \\
>21 \mathrm{~mm} \text { with decreased } \\
\text { inspiratory collapse } \\
\text { (<50\% with a sniff or } \\
<20 \% \text { with quiet } \\
\text { inspiration) }\end{array}$ \\
\hline $\begin{array}{l}\text { Flattening of the } \\
\text { interventricular } \\
\text { septum (left } \\
\text { ventricular } \\
\text { eccentricity index } \\
>1.1 \text { in systole } \\
\text { and/or diastole) }\end{array}$ & $\begin{array}{l}\text { Early diastolic } \\
\text { pulmonary } \\
\text { regurgitation velocity } \\
>2.2 \mathrm{~m} / \mathrm{sec}\end{array}$ & $\begin{array}{l}\text { Right atrial area } \\
\text { (end-systole) }>18 \mathrm{~cm}^{2}\end{array}$ \\
\hline & PA diameter $>25 \mathrm{~mm}$. & \\
\hline
\end{tabular}

PA: pulmonary artery. ${ }^{a}$ Echocardiographic signs from at least two different categories $(\mathrm{A} / \mathrm{B} / \mathrm{C})$ from the list should be present to alter the level of echocardiographic probability of pulmonary hypertension.

resonance (CMR) imaging to exclude sinus venosus atrial septal defect and/or anomalous pulmonary venous return. In cases of suspicion of LV diastolic dysfunction, Doppler echocardiographic signs should be assessed even if their reliability is considered low. RHC should be considered when the diagnosis remains uncertain after non-invasive investigations (see section 8.1). The practical clinical value of exercise Doppler echocardiography in the identification of cases with $\mathrm{PH}$ limited to exercise is uncertain because of the lack of validated criteria and prospective confirmatory data.

\subsubsection{Ventilation/perfusion lung scan}

A ventilation/perfusion (V/Q) lung scan should be performed in patients with $\mathrm{PH}$ to look for CTEPH. The V/Q scan has been the screening method of choice for CTEPH because of its higher sensitivity

TABLE 9 Diagnostic management suggested according to echocardiographic probability of pulmonary hypertension in patients with symptoms compatible with pulmonary hypertension, with or without risk factors for pulmonary arterial hypertension or chronic thromboembolic pulmonary hypertension

\begin{tabular}{|c|c|c|c|c|c|c|c|}
\hline $\begin{array}{c}\text { Echocardiographic } \\
\text { probability of PH }\end{array}$ & $\begin{array}{l}\text { Without risk factors or associated } \\
\text { condition for PAH or CTEPH d }\end{array}$ & Classa & Levelb & $\begin{array}{l}\text { With risk factors or associated } \\
\text { conditions for PAH or CTEPHc }\end{array}$ & Classa & Levelb & Refc \\
\hline Low & $\begin{array}{l}\text { Alternative diagnosis should be } \\
\text { considered }\end{array}$ & Ila & C & $\begin{array}{l}\text { Echo follow-up should be } \\
\text { considered }\end{array}$ & Ila & C & \\
\hline \multirow{2}{*}{ Intermediate } & $\begin{array}{l}\text { Alternative diagnosis, echo } \\
\text { follow-up, should be considered }\end{array}$ & Ila & \multirow{2}{*}{$C$} & \multirow{2}{*}{$\begin{array}{c}\text { Further assessment of } \mathrm{PH} \\
\text { including } \mathrm{RHC} \text { should be } \\
\text { considerede }\end{array}$} & \multirow{2}{*}{ Ila } & \multirow{2}{*}{ B } & \multirow{2}{*}[45,46]{} \\
\hline & $\begin{array}{l}\text { Further investigation of } \mathrm{PH} \text { may be } \\
\text { considerede }\end{array}$ & Ilb & & & & & \\
\hline High & $\begin{array}{l}\text { Further investigation of } \mathrm{PH} \\
\text { (including } \mathrm{RHC} \text { ) is recommended }\end{array}$ & 1 & C & $\begin{array}{l}\text { Further investigation of } \mathrm{PH} \\
\text { including } \mathrm{RHC} \text { is recommended }\end{array}$ & 1 & C & \\
\hline
\end{tabular}

CTEPH: chronic thromboembolic pulmonary hypertension; Echo: echocardiographic; $\mathrm{PAH}$ : pulmonary arterial hypertension; $\mathrm{PH}$ : pulmonary hypertension; RHC: right heart catheterization. ${ }^{a}$ Class of recommendation. ${ }^{b}$ Level of evidence. ${ }^{\mathrm{C}}$ Referencels) supporting recommendations.

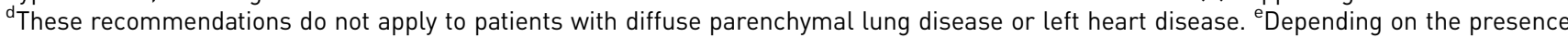
of risk factors for $\mathrm{PH}$ group 2, 3 or 5. Further investigation strategy may differ depending on whether risk factors/associated conditions suggest higher probability of PAH or CTEPH - see diagnostic algorithm. 
compared with CT pulmonary angiogram (CTPA), especially in inexperienced centres [47]. A normal- or low-probability V/Q scan effectively excludes CTEPH with a sensitivity of $90-100 \%$ and a specificity of 94-100\%; however, many V/Q scans are not diagnostic. While in PAH the V/Q lung scan may be normal, it may also show small peripheral unmatched and non-segmental defects in perfusion. A caveat is that unmatched perfusion defects may also be seen in other pulmonary vascular disease such as PVOD. While a V/Q scan is still recommended as the screening test of choice, ventilation scans are often replaced with either a recent chest radiograph or a recent high-resolution CT of the lungs, but such practices are not really evidence-based. Also, CT is preferred in many centres since it is more readily available. A few studies suggest that single photon emission CT, also a nuclear medicine technique, could be superior to V/ $\mathrm{Q}$ planar scan and CTPA, but these results need more extensive evaluation [48]. More recently, newer techniques such as three-dimensional magnetic resonance (MR) perfusion mapping, have been demonstrated to be as sensitive as traditional perfusion scintigraphy in screening for CTEPH; MR can also be used as a radiation-free modality to assess both ventilation and perfusion in CTEPH [49].

\subsubsection{High-resolution computed tomography, contrast-enhanced computed tomography, and pulmonary angiography}

CT imaging is a widely available tool that can provide important information on vascular, cardiac, parenchymal and mediastinal abnormalities. It may suggest the diagnosis of PH (PA or RV enlargement), identify a cause of $\mathrm{PH}$ such as $\mathrm{CTEPH}$ or lung disease, provide clues as to the form of $\mathrm{PAH}$ (e.g. oesophageal dilation in SSc or congenital cardiac defects such as anomalous pulmonary venous drainage) and also provide prognostic information [50].

CT may raise a suspicion of $\mathrm{PH}$ in symptomatic patients or those examined for unrelated indications by showing an increased PA diameter $(\geqslant 29 \mathrm{~mm})$ and pulmonary:ascending aorta diameter ratio $(\geqslant 1.0)$. A segmental artery:bronchus ratio $>1: 1$ in three or four lobes has been reported to have high specificity for $\mathrm{PH}[51,52]$.

High-resolution CT provides detailed views of the lung parenchyma and facilitates the diagnosis of interstitial lung disease and emphysema. High-resolution CT may also be very helpful where there is a clinical suspicion of PVOD. Characteristic changes of interstitial oedema with diffuse central ground-glass opacification and thickening of interlobular septa support the diagnosis of PVOD; additional findings may include lymphadenopathy, pleural shadows and effusions [53]. Pulmonary capillary haemangiomatosis is suggested by diffuse bilateral thickening of the interlobular septa and the presence of small, centrilobular, poorly circumscribed nodular opacities. However, ground-glass abnormalities are also present in PAH, occurring in more than one-third of patients [50].

Contrast CT angiography of the PA is helpful in determining whether there is evidence of surgically accessible CTEPH. It can delineate the typical angiographic findings in CTEPH, such as complete obstruction, bands and webs and intimal irregularities, as accurately and reliably as digital subtraction angiography [54, 55]. With this technique, collaterals from bronchial arteries can be identified.

Traditional pulmonary angiography is required in most patients for the workup of CTEPH to identify those who may benefit from pulmonary endarterectomy (PEA) or BPA [56, 57]. Angiography can be performed safely by experienced staff in patients with severe $\mathrm{PH}$ using modern contrast media and selective injections. Angiography may also be useful in the evaluation of possible vasculitis or pulmonary arteriovenous malformations, but CT angiography has similar or even higher accuracy for both diagnoses, and is less invasive $[58,59]$.

\subsubsection{Cardiac magnetic resonance imaging}

CMR imaging is accurate and reproducible in the assessment of RV size, morphology and function and allows non-invasive assessment of blood flow, including stroke volume, CO, pulmonary arterial distensibility and RV mass.

In patients with suspected $\mathrm{PH}$, the presence of late gadolinium enhancement, reduced pulmonary arterial distensibility and retrograde flow have high predictive value for the identification of $\mathrm{PH}$; however, no single CMR measurement can exclude $\mathrm{PH}$ [60-62]. In patients with $\mathrm{PH}, \mathrm{CMR}$ may also be useful in cases of suspected CHD if echocardiography is not conclusive.

Contrast-enhanced and unenhanced MR angiography have a potential in the study of the pulmonary vasculature in patients with suspected $\mathrm{CTEPH}$, particularly in clinical scenarios such as suspected chronic embolism in pregnant women, young patients or when iodine-based contrast media injection is contraindicated [63].

CMR provides useful prognostic information in patients with PAH both at baseline and at follow-up [64-66]. 


\subsubsection{Blood tests and immunology}

Blood tests are not useful in diagnosing $\mathrm{PH}$, but are required to identify the aetiology of some forms of $\mathrm{PH}$ as well as end organ damage. Routine biochemistry, haematology and thyroid function tests are required in all patients, as well as a number of other specific blood tests. Liver function tests may be abnormal because of high hepatic venous pressure, liver disease and/or endothelin receptor antagonist (ERA) therapy. Hepatitis serology should be performed if clinical abnormalities are noted. Thyroid disease is common in PAH and may develop during the course of the disease. This should always be considered in cases of abrupt deterioration.

Serological testing is required to detect underlying CTD, hepatitis and human immunodeficiency virus (HIV). Up to $40 \%$ of patients with IPAH have elevated antinuclear antibodies usually in a low titre (1:80). It is important to look for evidence of SSc since this disease has a relatively high prevalence of PAH. Limited scleroderma typically has antinuclear antibodies, including anti-centromere, dsDNA, anti-Ro, U3-RNP, B23, Th/To and U1-RNP. Diffuse scleroderma is typically associated with a positive U3-RNP. Patients with systemic lupus erythematosus may have anticardiolipin antibodies.

Patients with CTEPH should undergo thrombophilia screening, including antiphospholipid antibodies, anticardiolipin antibodies and lupus anticoagulant. HIV testing is required in PAH. N-terminal pro-brain natriuretic peptide (NT-proBNP) may be elevated in patients with $\mathrm{PH}$ and is an independent risk predictor in these patients.

\subsubsection{Abdominal ultrasound scan}

Similar to blood tests, abdominal ultrasound may be useful for identification of some of the clinical entities associated with PAH. Abdominal ultrasound may confirm but not formally exclude portal hypertension. The use of contrast agents and the addition of a colour Doppler examination may improve the accuracy of the diagnosis [67]. Portal hypertension can be reliably confirmed or excluded by measurement of the gradient between free and occluded (wedge) hepatic vein pressure at the time of RHC [68].

\subsubsection{Right heart catheterization and vasoreactivity}

RHC is required to confirm the diagnosis of PAH and CTEPH, to assess the severity of haemodynamic impairment and to undertake vasoreactivity testing of the pulmonary circulation in selected patients (Table 10). When performed at expert centres, these procedures have low morbidity $(1.1 \%)$ and mortality $(0.055 \%)$ rates [69]. The threshold to perform left heart catheterization in addition to RHC should be low in patients with clinical risk factors for coronary artery disease or heart failure with preserved ejection fraction, as well as in patients with echocardiographic signs of systolic and/or diastolic LV dysfunction. Specific recommendations for catheterization of patients with LHD or lung disease in addition to Table 10 are described in Tables 31 and 33, respectively. Measurement of LV end-diastolic pressure is also important to avoid misclassification of patients with an elevated PAWP when this is unexpected and may be inaccurate [absence of risk factors for heart failure with preserved ejection fraction, normal left atrial (LA) size and absence of echocardiographic markers of elevated LV filling pressures].

The interpretation of invasive haemodynamics should be made in the context of the clinical picture and imaging, in particular echocardiography. Cardiac catheterization should be performed after the completion of other investigations so that it can answer specific questions that may arise from these investigations and avoid an unnecessary procedure where an alternative diagnosis is revealed.

RHC is a technically demanding procedure that requires meticulous attention to detail to obtain clinically useful information. To obtain high-quality results and to be of low risk to patients, the procedure should be limited to expert centres. Particular attention should be paid to the following issues:

- The external pressure transducer should be zeroed at the mid-thoracic line in a supine patient, halfway between the anterior sternum and the bed surface [70]. This represents the level of the LA.

- Pressure measurements should be made in the PA, PA wedge position, RV and RA. Where a balloon catheter is used, it should be inflated in the RA, from where the catheter should be advanced until it reaches the PAWP position. Repeated deflations and inflations of the balloon in the end pulmonary arteries should be avoided because this has been associated with rupture of the pulmonary arteries. The PAWP is a surrogate of LA pressure and should be recorded as the mean of three measurements. Blood sampling should also be considered with the balloon inflated in the wedge position to confirm that a true PAWP measurement has been taken, as this should have the same saturation as systemic blood. All pressure measurements should be determined at the end of normal expiration (breath holding is not required). Alternatively, assuming that negative inspiratory and positive expiratory intrathoracic pressures cancel each other out, averaging pulmonary vascular pressures over several respiratory cycles is also acceptable, except in dynamic hyperinflation states [70]. Ideally, high-fidelity tracings that can be 
printed on paper should be used rather than small moving traces on a cardiac monitor. Non-invasive blood pressure should be recorded at the time of the procedure if left heart catheterization is not undertaken.

- Blood samples for oximetry should be taken from the high superior vena cava, IVC and PA at a minimum. Systemic arterial blood oxygen $\left(\mathrm{O}_{2}\right)$ saturation should also be determined. A stepwise assessment of $\mathrm{O}_{2}$ saturation should be performed in every patient with a pulmonary arterial $\mathrm{O}_{2}$ saturation $>75 \%$ and whenever a left-to-right shunt is suspected.

- CO should be measured using thermodilution or the direct Fick method. Thermodilution measured in triplicate is the preferred method because it can provide reliable measurements even in patients with low $\mathrm{CO}$ and/or severe tricuspid regurgitation [71]. In patients with intracardiac shunts, thermodilution may be inaccurate because of early recirculation of the injectate. The direct Fick method requires direct measurement of $\mathrm{O}_{2}$ uptake, a technique that is not widely available. The indirect Fick method, which uses estimated values of $\mathrm{O}_{2}$ uptake, is acceptable but lacks reliability.

- Pulmonary vasoreactivity testing for identification of patients suitable for high-dose calcium channel blocker (CCB) treatment is recommended only for patients with IPAH, HPAH or drug-induced PAH. It should be performed at the time of RHC. In all other forms of PAH and PH the results can be misleading and responders are rare. Inhaled nitric oxide (NO) at 10-20 parts per million (ppm) is the standard of care for vasoreactivity testing, but i.v. epoprostenol, i.v. adenosine or inhaled iloprost can be used as alternatives (Web Table IV). A positive acute response is defined as a reduction of the mean $\mathrm{PAP} \geqslant 10 \mathrm{mmHg}$ to reach an absolute value of mean PAP $\leqslant 40 \mathrm{mmHg}$ with an increased or unchanged CO. Only about $10 \%$ of patients with IPAH will meet these criteria. The use of $\mathrm{CCBs}, \mathrm{O}_{2}$, phosphodiesterase type 5 inhibitors or other vasodilators for acute vasoreactivity testing is discouraged.

- Interpretation of the PAWP at a single point in time needs to be performed in a clinical context. In many patients with LHD, PAWP may be reduced to $<15 \mathrm{mmHg}$ with diuretics [72-74]. For this reason, the effect of an acute volume challenge on left heart filling pressures has been considered [75]. Limited data suggest that a fluid bolus of $500 \mathrm{ml}$ appears to be safe and may discriminate patients with PAH from those with LV diastolic dysfunction [76, 77]. Further evaluation of administering a fluid challenge is required before this can be considered for routine clinical practice. Similarly, exercise haemodynamics to identify patients with LV diastolic dysfunction is likely to be useful [2, 78, 79], but lacks standardisation and requires further evaluation [17]. Furthermore, PAWP may underestimate LV end-diastolic pressure [80].

- Derived variables calculated from the RHC measurements should include transpulmonary pressure gradient (TPG) and PVR. A PVR $>3 \mathrm{WU}$ is required for the diagnosis of PAH [1]. PVR is commonly used but has the disadvantage of being a composite variable that is highly sensitive to changes in both flow and filling pressure and may not reflect changes in the pulmonary circulation at rest [81, 82]. The DPG between the mean PAWP and diastolic PAP is less affected by flow and filling pressures [81] but may not be of prognostic value [83]. DPG may have a role in patients suspected of having PH related to LHD, as discussed in section 8 [4].

- Coronary angiography may be required in the presence of angina, risk factors for coronary artery disease and listing for PEA or lung transplantation. It may identify left main stem coronary artery compression by an enlarged PA as well as coronary artery disease.

Recommendations for right and left heart catheterization and vasoreactivity testing are summarised in the Tables 10 and 11 .

\subsubsection{Genetic testing}

The availability of molecular genetic diagnosis has opened up a new field for patient care, including genetic counselling for PAH (developed in section 6.3.1.8) [26]. Genetic testing and counselling follows strict local regulations that set the conditions for prescribing and conducting reviews of the genetic characteristics of a patient. The ethical principles are to inform patients properly to avoid harm, to allow patients to preserve their autonomy (disclosure about the process, risks and benefits of the genetic test without external pressures) and to allow equal access to genetic counselling and testing. Patients with sporadic or familial $\mathrm{PAH}$ or $\mathrm{PVOD} / \mathrm{PCH}$ should be advised about the availability of genetic testing and counselling because of the strong possibility that they carry a disease-causing mutation. Trained professionals should offer counselling and testing to the patient. Genetic counselling and BMPR2 mutation screening (point mutations and large rearrangements) should be offered by referral centres to patients with IPAH considered to be sporadic or induced by anorexigens and to patients with a family history of PAH. When no BMPR2 mutations are identified in familial PAH patients or in IPAH patients $<40$ years old, or when PAH occurs in patients with a personal or familial history of hereditary haemorrhagic telangiectasia, screening of the ACVRL1 and ENG genes may be performed. If no mutations in the $B M P R 2, A C V R L 1$ and $E N G$ genes are identified, screening of rare mutations may be considered (KCNK3, $C A V 1$, etc.). 
TABLE 10 Recommendations for right heart catheterization in pulmonary hypertension

\begin{tabular}{|c|c|c|c|}
\hline Recommendations & Class $^{a}$ & Level $^{\mathrm{b}}$ & Ref. $^{c}$ \\
\hline $\begin{array}{l}\text { RHC is recommended to confirm the } \\
\text { diagnosis of pulmonary arterial } \\
\text { hypertension (group 1) and to support } \\
\text { treatment decisions }\end{array}$ & I & C & \\
\hline $\begin{array}{l}\text { In patients with } \mathrm{PH} \text {, it is recommended to } \\
\text { perform } \mathrm{RHC} \text { in expert centres (see } \\
\text { section } 12 \text { ) as it is technically } \\
\text { demanding and may be associated with } \\
\text { serious complications }\end{array}$ & 1 & B & [69] \\
\hline $\begin{array}{l}\text { RHC should be considered in pulmonary } \\
\text { arterial hypertension (group 1) to assess } \\
\text { the treatment effect of drugs (Table 16) }\end{array}$ & Ila & C & \\
\hline $\begin{array}{l}\text { RHC is recommended in patients with } \\
\text { congenital cardiac shunts to support } \\
\text { decisions on correction (Table 24) }\end{array}$ & I & C & \\
\hline $\begin{array}{l}\text { RHC is recommended in patients with PH } \\
\text { due to left heart disease (group 2) or lung } \\
\text { disease (group } 3 \text { ) if organ transplantation } \\
\text { is considered }\end{array}$ & I & C & \\
\hline $\begin{array}{l}\text { When measurement of PAWP is } \\
\text { unreliable, left heart catheterization } \\
\text { should be considered to measure LVEDP }\end{array}$ & Ila & C & \\
\hline $\begin{array}{l}\text { RHC may be considered in patients with } \\
\text { suspected } \mathrm{PH} \text { and left heart disease or } \\
\text { lung disease to assist in the differential } \\
\text { diagnosis and support treatment } \\
\text { decisions }\end{array}$ & $\mathrm{Ilb}$ & $C$ & \\
\hline $\begin{array}{l}\text { RHC is indicated in patients with CTEPH } \\
\text { (group 4) to confirm the diagnosis and } \\
\text { support treatment decisions }\end{array}$ & I & C & \\
\hline
\end{tabular}

CTEPH: chronic thromboembolic pulmonary hypertension; LVEDP: left ventricular end-diastolic pressure; PAWP: pulmonary artery wedge pressure; $\mathrm{PH}$ : pulmonary hypertension; $\mathrm{RHC}$ : right heart catheterization. ${ }^{a}$ Class of recommendation. ${ }^{b}$ Level of evidence. ${ }^{\mathrm{C}}$ Referencels) supporting recommendations.
TABLE 11 Recommendations for vasoreactivity testing

\begin{tabular}{|c|c|c|c|}
\hline Recommendations & Class ${ }^{a}$ & Level $^{b}$ & Ref.c $^{c}$ \\
\hline $\begin{array}{l}\text { Vasoreactivity testing is indicated only in } \\
\text { expert centres }\end{array}$ & I & C & [69] \\
\hline $\begin{array}{l}\text { Vasoreactivity testing is recommended in } \\
\text { patients with IPAH, HPAH and PAH } \\
\text { associated with drugs use to detect } \\
\text { patients who can be treated with high } \\
\text { doses of a CCB }\end{array}$ & I & C & {$[84,85]$} \\
\hline $\begin{array}{l}\text { A positive response to vasoreactivity } \\
\text { testing is defined as a reduction of mean } \\
\text { PAP } \geqslant 10 \mathrm{mmHg} \text { to reach an absolute } \\
\text { value of mean PAP } \leqslant 40 \mathrm{mmHg} \text { with an } \\
\text { increased or unchanged cardiac output }\end{array}$ & I & C & {$[85,86]$} \\
\hline $\begin{array}{l}\text { Nitric oxide is recommended forperforming } \\
\text { vasoreactivity testing }\end{array}$ & I & C & {$[85,86]$} \\
\hline $\begin{array}{l}\text { Intravenous epoprostenol is recommended } \\
\text { for performing vasoreactivity testing as } \\
\text { an alternative }\end{array}$ & I & C & {$[85,86]$} \\
\hline $\begin{array}{l}\text { Adenosine should be considered for } \\
\text { performing vasoreactivity testing as an } \\
\text { alternative }\end{array}$ & Ila & C & {$[87,88]$} \\
\hline $\begin{array}{l}\text { Inhaled iloprost may be considered for } \\
\text { performing vasoreactivity testing as an } \\
\text { alternative }\end{array}$ & $\mathrm{Ilb}$ & C & {$[89,90]$} \\
\hline $\begin{array}{l}\text { The use of oral or intravenous CCBs in } \\
\text { acute vasoreactvity testing is not } \\
\text { recommended }\end{array}$ & III & C & \\
\hline $\begin{array}{l}\text { Vasoreactivity testing to detect patients } \\
\text { who can be safely treated with high doses } \\
\text { of a CCB is not recommended in patients } \\
\text { with PAH other than IPAH, HPAH and PAH } \\
\text { associated with drugs use and is not } \\
\text { recommended in PH groups } 2,3,4 \text { and } 5\end{array}$ & III & C & \\
\hline
\end{tabular}

CCB: calcium channel blocker; HPAH: heritable pulmonary arterial hypertension; IPAH: idiopathic pulmonary arterial hypertension; PAP: pulmonary arterial pressure; $\mathrm{PAH}$ : pulmonary arterial hypertension. ${ }^{\mathrm{a}}$ Class of recommendation. ${ }^{\mathrm{b}}$ Level of evidence. ${ }^{c}$ Reference(s) supporting recommendations.

Patients with sporadic or familial PVOD/PCH should be tested for EIF2AK4 mutations [28]. The presence of a bi-allelic EIF2AK4 mutation is sufficient to confirm a diagnosis of PVOD/PCH without performing a hazardous lung biopsy for histological confirmation.

\subsection{Diagnostic algorithm}

The diagnostic algorithm is shown in Figure 1: the diagnostic process starts after the suspicion of $\mathrm{PH}$ and echocardiography compatible with $\mathrm{PH}$ (according to the different levels of $\mathrm{PH}$ probability reported in Tables 8 and 9) and continues with the identification of the more common clinical groups of $\mathrm{PH}$ [group 2 (LHD) and group 3 (lung diseases)], then distinguishes group 4 (CTEPH) and finally makes the diagnosis and recognizes the different types in group $1(\mathrm{PAH})$ and the rarer conditions in group 5.

PAH should be considered in the differential diagnosis of exertional dyspnoea, syncope, angina and/or progressive limitation of exercise capacity, particularly in patients without apparent risk factors, symptoms or signs of common cardiovascular and respiratory disorders. Special awareness should be directed towards 
patients with associated conditions and/or risk factors for the development of $\mathrm{PAH}$, such as family history, CTD, CHD, HIV infection, portal hypertension or a history of drug or toxin intake known to induce PAH (Table 7). In everyday clinical practice such awareness may be low. More often $\mathrm{PH}$ is found unexpectedly on transthoracic echocardiography requested for another indication.

If transthoracic echocardiography is compatible with a high or intermediate probability of $\mathrm{PH}$ (Table 9), a clinical history, symptoms, signs, ECG, chest radiograph, pulmonary function tests (PFTs, including DLCO, arterial blood gases analysis and nocturnal oximetry, if required) and high-resolution CT of the chest are requested to identify the presence of group 2 (LHD) or group 3 (lung diseases) PH. In case of an echocardiographic low probability of $\mathrm{PH}$ (Table 9), no additional investigations are required and other causes for the symptoms should be considered together with follow-up. If the diagnosis of left heart or lung diseases is confirmed, the appropriate treatment for these conditions should be considered. In the presence of severe $\mathrm{PH}$ and/or RV dysfunction, the patient should be referred to a $\mathrm{PH}$ expert centre where additional causes of $\mathrm{PH}$ can be explored. If the diagnosis of left heart or lung diseases is not confirmed, a V/Q lung scan should be performed for the differential diagnosis between CTEPH and PAH. Concurrently the patient should be referred to a $\mathrm{PH}$ expert centre.

If the V/Q scan shows multiple segmental perfusion defects, a diagnosis of group 4 (CTEPH) PH should be suspected [91]. The final diagnosis of CTEPH (and the assessment of suitability for PEA) will require CT pulmonary angiography, RHC and selective pulmonary angiography. The CT scan may also show signs suggestive of group 1' (PVOD). If a V/Q scan is normal or shows only subsegmental 'patchy' perfusion defects, a diagnosis of group $1(\mathrm{PAH})$ or the rarer conditions of group 5 should be considered. In Table 9, further management according to the probability of $\mathrm{PH}$ is given, including indications for RHC. Additional specific diagnostic tests, including haematology, biochemistry, immunology, serology, ultrasonography and genetics, will allow the final diagnosis to be refined.

Open or thoracoscopic lung biopsy entails a substantial risk of morbidity and mortality [92]. Because of the low likelihood of altering the diagnosis and treatment, biopsy is not recommended in PAH patients.

The recommendations for a diagnostic strategy are reported in the Table 12.

The pulmonary arterial hypertension screening programme is reported in the Web Addenda.

\section{Pulmonary arterial hypertension (group 1)}

\subsection{Clinical characteristics}

The clinical characteristics of PAH are not specific and can be derived from the general description reported in section 5.1.1. More detailed descriptions of the individual PAH subsets are reported in the section 7.

\subsection{Evaluation of severity}

\subsubsection{Clinical parameters, imaging and haemodynamics}

Clinical assessment remains a key part of the evaluation of patients with $\mathrm{PH}$, as it provides valuable information for determining disease severity, improvement, deterioration or stability. Elementary parts of history taking between follow-up visits include changes in exercise capacity, episodes of chest pain, arrhythmia, haemoptysis or syncope and changes in medications, as well as adherence to the prescribed drugs. Physical examination provides information on the presence or absence of peripheral or central cyanosis, enlarged jugular veins, oedema, ascites and pleural effusions and on heart rate, rhythm and blood pressure.

The World Health Organization functional class (WHO-FC) (Web Table V), despite its interobserver variability [95], remains one of the most powerful predictors of survival, not only at diagnosis, but also during follow-up [96-98]. A worsening FC is one of the most alarming indicators of disease progression, which should trigger further diagnostic studies to identify the causes of clinical deterioration [97, 99].

As RV function is a key determinant of exercise capacity and outcome in patients with PH, echocardiography remains an important follow-up tool. In contrast to common belief, the estimated systolic PAP (PAPs) at rest is usually not prognostic and not relevant for therapeutic decision making [96, 97, 100]. An increase in PAPs does not necessarily reflect disease progression and a decrease in PAPs does not necessarily signal improvement. A comprehensive echocardiographic assessment includes a description of chamber sizes, particularly of the RA and RV area, the magnitude of tricuspid regurgitation, the LV eccentricity index and RV contractility, which can be determined by several variables, including RV longitudinal systolic strain/strain rate and RV fractional area change, Tei index and tricuspid annular plane systolic excursion (TAPSE) [101-108].

Three-dimensional echocardiography may achieve a better estimation than standard two-dimensional assessment, but underestimations of volumes and ejection fractions have been reported [109].

Speckle tracking improves the quantification of RV function [110]. Given the complex geometry of the RV, none of these variables alone is sufficient to describe RV function, and the overall impression of an 
TABLE 12 Recommendations for diagnostic strategy

\begin{tabular}{|c|c|c|c|}
\hline Recommendations & Class $^{a}$ & Levelb & Ref.c \\
\hline $\begin{array}{l}\text { Echocardiography is recommended as a } \\
\text { first-line non-invasive diagnostic } \\
\text { investigation in case of suspicion of } \mathrm{PH}\end{array}$ & 1 & C & \\
\hline $\begin{array}{l}\text { Ventilation/perfusion or perfusion lung } \\
\text { scan is recommended in patients with } \\
\text { unexplained } \mathrm{PH} \text { to exclude CTEPH }\end{array}$ & 1 & C & [47] \\
\hline $\begin{array}{l}\text { Contrast CT angiography of the PA is } \\
\text { recommended in the workup of patients } \\
\text { with CTEPH }\end{array}$ & I & C & [93] \\
\hline $\begin{array}{l}\text { Routine biochemistry. haematology, } \\
\text { immunology, HIV testing and thyroid } \\
\text { function tests are recommended in all } \\
\text { patients with PAH to identify the specific } \\
\text { associated condition }\end{array}$ & 1 & C & \\
\hline $\begin{array}{l}\text { Abdominal ultrasound is recommended } \\
\text { for the screening of portal hypertension }\end{array}$ & 1 & C & [67] \\
\hline $\begin{array}{l}\text { Lung function test with DLCO is } \\
\text { recommended in the initial evaluation of } \\
\text { patients with } \mathrm{PH}\end{array}$ & 1 & C & [36] \\
\hline $\begin{array}{l}\text { High-resolution CT should be considered } \\
\text { in all patients with } \mathrm{PH}\end{array}$ & Ila & C & [94] \\
\hline $\begin{array}{l}\text { Pulmonary angiography should be } \\
\text { considered in the workup of patients with } \\
\text { CTEPH }\end{array}$ & Ila & C & \\
\hline $\begin{array}{l}\text { Open or thoracoscopic lung biopsy is not } \\
\text { recommended in patients with PAH }\end{array}$ & III & C & \\
\hline
\end{tabular}

CT: computed tomography; CTEPH: chronic thromboembolic pulmonary hypertension; DLCO: diffusing capacity of the lung for carbon monoxide; $\mathrm{PAH}$ : pulmonary arterial hypertension; $\mathrm{PH}$ : pulmonary hypertension. ${ }^{\mathrm{a}}$ Class of recommendation. ${ }^{\mathrm{b}}$ Level of evidence. ${ }^{\mathrm{C}}$ Reference(s) supporting recommendations.

experienced physician is often more important than single variables. Echocardiography during exercise provides additional information on RV function. Of note, a marked increase $(>30 \mathrm{mmHg}$ ) of PAPs during exercise reflects better RV function and is associated with a better long-term outcome than a modest or no increase [111]. This so-called contractile reserve has recently been shown to be an independent prognostic marker in patients with severe $\mathrm{PH}[111]$.

CMR imaging is more accurate for the assessment of RV morphology and function than echocardiography and also allows measurement of stroke volume and CO. A number of CMR prognostic markers have been identified, including increased RV volume, reduced LV volume, reduced RV ejection fraction and reduced stroke volume. There is some evidence that follow-up CMR studies may have utility in the long-term management of PAH by identifying RV failure prior to the development of clinical features $[64,66,112,113]$.

Haemodynamics assessed by RHC provide important prognostic information, both at the time of diagnosis and during follow-up. RA pressure, cardiac index (CI) and mixed venous oxygen saturation $\left(\mathrm{SvO}_{2}\right)$ are the most robust indicators of RV function and prognosis, whereas PAPm provides little prognostic information (except for CCB responders) [96, 97, 99, 100, 114]. Non-invasive assessment of CO by rebreathing techniques [71] or bioreactance [115] has not yet been sufficiently validated to allow routine clinical use and therapeutic decision making.

There are still uncertainties around the optimal timing of follow-up RHC. Strategies vary between centres, from regular invasive haemodynamic assessments to a predominantly non-invasive follow-up strategy. 
There is no evidence that an approach involving regular RHC is associated with better outcomes than a predominantly non-invasive follow-up strategy. However, there is consensus among experts that RHC should be performed whenever therapeutic decisions can be expected from the results, which may include changes in medications and/or decisions regarding listing for transplantation.

\subsubsection{Exercise capacity}

The 6-minute walking test (6MWT), a submaximal exercise test, remains the most widely used exercise test in $\mathrm{PH}$ centres. The test is easy to perform, inexpensive and familiar to patients and centres. As with all $\mathrm{PH}$ assessments, 6MWT results must always be interpreted in the clinical context. The 6-minute walking distance (6MWD) is influenced by several factors, including sex, age, height, weight, co-morbidities, need for $\mathrm{O}_{2}$, learning curve and motivation. Nevertheless, test results are usually given in absolute numbers rather than percent predicted. Absolute values, but not changes in $6 \mathrm{MWD}$, provide prognostic information, but there is no single threshold that is applicable for all patients (see below) $[96,99,116-118]$. It is recommended to use the Borg score at the end of the 6MWT to determine the level of effort. In addition, some studies suggest that adding peripheral $\mathrm{O}_{2}$ measurements and heart rate response may improve the prognostic relevance, but these findings await independent confirmation $[119,120]$.

Cardiopulmonary exercise testing (CPET) is usually performed as a maximal exercise test and provides important information on exercise capacity as well as on gas exchange, ventilator efficacy and cardiac function during exercise. Most $\mathrm{PH}$ centres use an incremental ramp protocol, although the test has not yet been standardized for this patient population. Patients with PAH show a typical pattern with a low end-tidal partial pressure of carbon dioxide $\left(\mathrm{pCO}_{2}\right)$, high ventilator equivalents for carbon dioxide (VE/ $\left.\mathrm{VCO}_{2}\right)$, low oxygen pulse $\left(\mathrm{VO}_{2} / \mathrm{HR}\right)$ and low peak oxygen uptake (peak $\left.\mathrm{VO}_{2}\right)$ [121]. Several variables determined by CPET provide prognostic information, although peak $\mathrm{VO}_{2}$ is most widely used for therapeutic decision making [106, 122-125]. The diagnostic and prognostic information provided by CPET add to that provided by the 6MWT [122].

\subsubsection{Biochemical markers}

There is still no specific marker for PAH or pulmonary vascular remodelling, although a wide variety of biomarkers have been explored in the field. These can be grouped into markers of vascular dysfunction [asymmetric dimethylarginine (ADMA), endothelin-1, angiopoeitins, von Willebrand factor] [126-131], markers of inflammation (C-reactive protein, interleukin 6, chemokines) [132-135], markers of myocardial stress (atrial natriuretic peptide, brain natriuretic peptide (BNP)/NT-proBNP, troponins) [97, 118, 136-139], markers of low $\mathrm{CO}$ and/or tissue hypoxia $\left[\mathrm{pCO}_{2}\right.$, uric acid, growth differentiation factor 15 (GDF15), osteopontin] [38, 140-142] and markers of secondary organ damage (creatinine, bilirubin) $[97,137]$. This list is constantly growing, but so far BNP and NT-proBNP remain the only biomarkers that are widely used in the routine practice of $\mathrm{PH}$ centres as well as in clinical trials. BNP/NT-proBNP levels correlate with myocardial dysfunction and provide prognostic information at the time of diagnosis and during follow-up assessments [143]. They are not specific for PH, but can be elevated in almost any heart disease. BNP/NT-proBNP levels tend to have a high variability and should be interpreted in the clinical context. There are no clear advantages of using BNP versus NT-proBNP. BNP appears to have a slightly tighter correlation with pulmonary haemodynamics and is less affected by kidney function, whereas NT-proBNP seems to be a stronger predictor of prognosis [137].

\subsubsection{Comprehensive prognostic evaluation and risk assessment}

Regular assessment of patients with $\mathrm{PAH}$ in expert $\mathrm{PH}$ centres is strongly recommended. A comprehensive assessment is required since there is no single variable that provides sufficient diagnostic and prognostic information. The most important questions to be addressed at each visit are (i) is there any evidence of clinical deterioration since the last assessment?; (ii) if so, is clinical deterioration caused by progression of $\mathrm{PH}$ or by a concomitant illness?; (iii) is RV function stable and sufficient?; and (iv) is the current status compatible with a good long-term prognosis, i.e. does the patient meet the low-risk criteria (see below)?

In order to answer these questions, a multidimensional approach is needed. Table 13 lists the variables that are most frequently used in $\mathrm{PH}$ centres. Not all of them need to be assessed at each visit. However, the basic programme should include determination of the FC and at least one measurement of exercise capacity, e.g. $6 \mathrm{MWD}$ or CPET. It is also recommended to obtain some information on RV function, either by measuring BNP/NT-proBNP or by performing echocardiography. Most of the proposed variables and cut-off values are based on expert opinion. They may provide prognostic information and may be used to guide therapeutic decisions, but application to individual patients must be done carefully. The indicated mortality rates are crude estimates and the depicted variables have been studied mostly in patients with IPAH. Not all variables may be in the same risk group, and it is the comprehensive assessment of individual patients that should guide treatment decisions. The individual risk is further modified by other 
TABLE 13 Risk assessment in pulmonary arterial hypertension

\begin{tabular}{|c|c|c|c|}
\hline $\begin{array}{l}\text { Determinants of prognosisa } \\
\text { (estimated 1-year mortality) }\end{array}$ & Low risk $<5 \%$ & Intermediate risk 5-10\% & High risk $>10 \%$ \\
\hline Clinical signs of right heart failure & Absent & Absent & Present \\
\hline Progression of symptoms & No & Slow & Rapid \\
\hline Syncope & No & Occasional syncopeb & Repeated syncopec \\
\hline WHO functional class & I, II & III & IV \\
\hline 6MWD & $>440 \mathrm{~m}$ & $165-440 \mathrm{~m}$ & $<165 \mathrm{~m}$ \\
\hline Cardiopulmonary exercise testing & $\begin{array}{c}\text { Peak } \mathrm{VO}_{2}>15 \mathrm{ml} / \mathrm{min} / \mathrm{kg} \\
(>65 \% \text { pred.) } \\
\text { VE } / \mathrm{VCO}_{2} \text { slope }<36\end{array}$ & $\begin{array}{c}\text { Peak } \mathrm{VO}_{2} 11-15 \mathrm{ml} / \mathrm{min} / \mathrm{kg} \\
(35-65 \% \text { pred.) } \\
\text { VE/ } / \mathrm{VCO}_{2} \text { slope } 36-44.9\end{array}$ & $\begin{array}{c}\text { Peak } \mathrm{VO}_{2}<11 \mathrm{ml} / \mathrm{min} / \mathrm{kg} \\
{[<35 \% \text { pred.) }} \\
\text { VE/VCO } 2 \text { slope } \geqslant 45\end{array}$ \\
\hline NT-proBNP plasma levels & $\begin{array}{c}\text { BNP }<50 \mathrm{ng} / \mathrm{l} \\
\text { NT-proBNP }<300 \mathrm{ng} / \mathrm{l}\end{array}$ & $\begin{array}{c}\text { BNP 50-300 ng/l } \\
\text { NT-proBNP } 300-1400 \mathrm{ng} / \mathrm{l}\end{array}$ & $\begin{array}{c}\mathrm{BNP}>300 \mathrm{ng} / \mathrm{l} \\
\text { NT-proBNP }>1400 \mathrm{ng} / \mathrm{l}\end{array}$ \\
\hline Imaging (echocardiography, CMR imaging) & $\begin{array}{c}\mathrm{RA} \text { area }<18 \mathrm{~cm}^{2} \\
\text { No pericardial effusion }\end{array}$ & $\begin{array}{l}\text { RA area } 18-26 \mathrm{~cm}^{2} \\
\text { No or minimal, } \\
\text { pericardial effusion }\end{array}$ & $\begin{array}{l}\text { RA area }>26 \mathrm{~cm}^{2} \\
\text { pericardial effusion }\end{array}$ \\
\hline Haemodynamics & $\begin{array}{c}\mathrm{RAP}<8 \mathrm{mmHg} \\
\mathrm{Cl} \geqslant 2.5 \mathrm{I} / \mathrm{min} / \mathrm{m}^{2} \\
\mathrm{SvO}_{2}>65 \%\end{array}$ & $\begin{array}{c}\mathrm{RAP} 8-14 \mathrm{mmHg} \\
\mathrm{Cl} 2.0-2.4 \mathrm{l} / \mathrm{min} / \mathrm{m}^{2} \\
\mathrm{SvO}_{2} 60-65 \%\end{array}$ & $\begin{array}{l}\mathrm{RAP}>14 \mathrm{mmHg} \\
\mathrm{Cl}<2.0 \mathrm{l} / \mathrm{min} / \mathrm{m}^{2} \\
\mathrm{SvO}_{2}<60 \%\end{array}$ \\
\hline
\end{tabular}

6MWD: 6-minute walking distance; BNP: brain natriuretic peptide; $\mathrm{Cl}$ : cardiac index; CMR: cardiac magnetic resonance; NT-proBNP: $\mathrm{N}$-terminal pro-brain natriuretic peptide; pred.: predicted; RA: right atrium; RAP: right atrial pressure; $\mathrm{SvO}_{2}$ : mixed venous oxygen saturation; $\mathrm{VE} / \mathrm{VCO}_{2}$ : ventilatory equivalents for carbon dioxide; $\mathrm{VO}_{2}$ : oxygen consumption; WHO: World Health Organization. ${ }^{\mathrm{a}}$ Most of the proposed variables and cut-off values are based on expert opinion. They may provide prognostic information and may be used to guide therapeutic decisions, but application to individual patients must be done carefully. One must also note that most of these variables have been validated mostly for IPAH and the cut-off levels used above may not necessarily apply to other forms of PAH. Furthermore, the use of approved therapies and their influence on the variables should be considered in the evaluation of the risk. ${ }^{b}$ Occasional syncope during brisk or heavy exercise, or occasional orthostatic syncope in an otherwise stable patient. ' $R$ Repeated episodes of syncope, even with little or regular physical activity.

factors, such as the rate of disease progression and the presence or absence of signs of right heart failure, or syncope, and also by co-morbidities, age, sex, background therapy, and PAH subtype, among others.

Finally, the assessment of $\mathrm{PAH}$ patients should provide information on co-morbidities and disease complications. ECGs should be obtained on a regular basis to detect clinically relevant arrhythmias, which occur frequently in this patient population [33]. Patients with $\mathrm{PAH}$ occasionally present with progressive hypoxaemia and may be candidates for long-term $\mathrm{O}_{2}$ therapy. In addition, a low $\mathrm{PaCO}_{2}$ is associated with reduced pulmonary blood flow and has prognostic implications [38]. Thus arterial or capillary blood gases provide important information and should be part of the regular clinical assessment, at least in cases of clinical deterioration. Alternatively the peripheral $\mathrm{O}_{2}$ saturation may be used, but it is less reliable and does not provide information on $\mathrm{PaCO}_{2}$. The recommended basic laboratory workup (in addition to BNP/NT-proBNP) includes blood counts and international normalized ratio (INR) (in patients receiving vitamin $\mathrm{K}$ antagonists), as well as serum sodium, potassium, creatinine, uric acid, aspartate aminotransferase (ASAT), alanine aminotransferase (ALAT) (in patients receiving ERAs) and bilirubin. In addition, troponin, uric acid, iron status and thyroid function should be checked at least once a year or whenever the patient presents with clinical worsening. Tables 14 and 15 provide detailed recommendations on the follow-up assessment of patients with PAH.

\subsubsection{Definition of patient status}

Based on the comprehensive assessment described in the last section, the patient can be classified as low risk, intermediate risk or high risk for clinical worsening or death (Table 13). Of course, there are several other factors that have an impact on disease manifestation and prognosis that cannot be affected by PAH therapy, including age, sex, underlying disease and co-morbidities. Although reliable individual predictions are always difficult, patients categorized as low risk have an estimated 1 -year mortality $<5 \%$. Basically these patients present with non-progressive disease in WHO-FC I or II with a $6 \mathrm{MWD}>440 \mathrm{~m}$ and no signs of clinically relevant RV dysfunction. The estimated 1-year mortality in the intermediate-risk group is $5-10 \%$. These patients typically present in WHO-FC III, with moderately impaired exercise capacity and signs of 
TABLE 14 Suggested assessment and timing for the follow-up of patients with pulmonary arterial hypertension

\begin{tabular}{|l|c|c|c|c|c|}
\hline & At baseline & $\begin{array}{c}\text { Every 3-6 } \\
\text { months }\end{array}$ & $\begin{array}{c}\text { Every 6-12 } \\
\text { months }\end{array}$ & $\begin{array}{c}3-6 \text { months } \\
\text { after changes } \\
\text { in therapya }\end{array}$ & $\begin{array}{c}\text { In case of } \\
\text { clinical } \\
\text { worsening }\end{array}$ \\
\hline $\begin{array}{l}\text { Medical assessment and } \\
\text { determination of functional class }\end{array}$ & + & + & + & + & + \\
\hline ECG & + & + & + & + & + \\
\hline 6MWT/Borg dyspnoea score & + & + & + & + & + \\
\hline CPET & + & + & + & + & + \\
\hline Echo & + & + & + & + & + \\
\hline Basic labb & + & + & + & + & + \\
\hline Extended labc & + & & + & + & + \\
\hline Blood gas analysis & + & & + & + & + \\
\hline Right heart catheterization & + & & $+{ }^{\mathrm{f}}$ & + \\
\hline
\end{tabular}

ALAT: alanine aminotransferase; ASAT: aspartate aminotransferase; BGA: blood gas analysis; BNP: brain natriuretic peptide; CPET: cardiopulmonary exercise testing; Echo: echocardiography; ECG: electrocardiogram; ERAs: endothelin receptor antagonists; FC: functional class; INR: international normalized ratio; lab: laboratory assessment; NT-proBNP: N-terminal pro-brain natriuretic peptide; RHC: right heart catheterization; TSH: thyroid stimulating hormone; 6MWT: 6-minute walking test. antervals to be adjusted according to patient needs. ${ }^{\mathrm{b}}$ Basic lab includes blood count, INR (in patients receiving vitamin $\mathrm{K}$ antagonists), serum creatinine, sodium, potassium, ASAT/ALAT (in patients receiving ERAs), bilirubin and BNP/NT-proBNP. ' Extended lab includes TSH, troponin, uric acid, iron status (iron, ferritin, soluble transferrin receptor) and other variables according to individual patient needs. ${ }^{\mathrm{d}}$ From arterial or arterialized capillary blood; may be replaced by peripheral oxygen saturation in stable patients or if BGA is not available. "Should be considered. 'Some centres perform RHCs at regular intervals during follow-up.

RV dysfunction, but not with RV failure. Patients in the high-risk group have an estimated 1-year mortality $>10 \%$. These patients present in WHO-FC III or IV with progressive disease and signs of severe RV dysfunction, or with RV failure and secondary organ dysfunction.

The variables shown in Table 13 may not behave consistently, i.e. they may fall into different risk categories. Again, it is the overall assessment that should drive therapeutic decisions.

\subsubsection{Treatment goals and follow-up strategy}

The overall treatment goal in patients with PAH is achieving a low-risk status (Table 13), which is usually associated with good exercise capacity, good quality of life, good RV function and a low mortality risk. Specifically, this means bringing and/or keeping the patient in WHO-FC II whenever possible. In most patients, this will be accompanied by a near-normal or normal 6MWD. Several treatment goals for the 6 MWD have been proposed including $>380 \mathrm{~m},>440 \mathrm{~m}$ and $>500 \mathrm{~m}[96,99,116-118,144]$. All of these numbers are based on survival analyses from selected cohorts or on expert opinion. The present guidelines adopt a threshold of $>440 \mathrm{~m}$ as suggested during the 5th World Symposium on Pulmonary Hypertension [145], because this number was derived from the largest cohort investigated so far [99]. Nevertheless, individual factors must be considered and lower values may be acceptable in elderly patients or patients with co-morbidities, whereas even values $>440 \mathrm{~m}$ may not be sufficient in younger, otherwise healthy patients. Especially in those patients, CPET should be regularly used, as it provides more objective information on exercise capacity and RV performance.

It should be noted that these treatment goals are not always realistic and may not be achievable in patients with advanced disease, patients with severe co-morbidities or very old patients.

\subsection{Therapy}

The therapy for PAH patients has evolved progressively in the past decade, increasing in complexity and in evidence for efficacy [146-148]. The treatment process of PAH patients cannot be considered as a mere 
TABLE 15 Recommendations for evaluation of the severity of pulmonary arterial hypertension and clinical response to therapy

\begin{tabular}{|l|c|c|c|}
\hline Recommendations & Class & Level $^{\mathrm{b}}$ & Ref.c \\
\hline $\begin{array}{l}\text { It is recommended to evaluate the } \\
\text { severity of PAH patients with a panel of } \\
\text { data derived from clinical assessment, } \\
\text { exercise tests, biochemical markers and } \\
\text { echocardiographic and haemodynamic } \\
\text { evaluations (Tables 13 and 14] }\end{array}$ & I & C & $\begin{array}{c}{[96,97,} \\
99]\end{array}$ \\
\hline $\begin{array}{l}\text { It is recommended to perform regular } \\
\text { follow-up assessments every 3-6 months } \\
\text { in stable patients (Table 14) }\end{array}$ & I & C & [98] \\
\hline $\begin{array}{l}\text { Achievement/maintenance of a low-risk } \\
\text { profile (Table 13) is recommended as an } \\
\text { adequate treatment response for } \\
\text { patients with PAH }\end{array}$ & I & C & [96-99] \\
\hline $\begin{array}{l}\text { Achievement/maintenance of an } \\
\text { intermediate-risk profile (Table 13) } \\
\text { should be considered an inadequate } \\
\text { treatment response for most patients } \\
\text { with PAH }\end{array}$ & Ila & C & [96-99] \\
\hline
\end{tabular}

$\mathrm{PAH}$ : pulmonary arterial hypertension. ${ }^{\mathrm{a}}$ Class of recommendation. ${ }^{\mathrm{b}}$ Level of evidence. ${ }^{\mathrm{c}}$ Referencels) supporting recommendations.
TABLE 16 Recommendations for general measures

\begin{tabular}{|c|c|c|c|}
\hline Recommendations & Class $^{\mathrm{a}}$ & Level $^{\mathrm{b}}$ & Ref. $^{\mathrm{c}}$ \\
\hline $\begin{array}{c}\text { It is recommended that PAH patients } \\
\text { avoid pregnancy }\end{array}$ & I & C & $\begin{array}{c}{[160,} \\
161]\end{array}$ \\
\hline $\begin{array}{c}\text { Immunization of PAH patients against } \\
\text { influenza and pneumococcal infection is } \\
\text { recommended }\end{array}$ & I & C & \\
\hline $\begin{array}{l}\text { Psychosocial support is recommended in } \\
\text { PAH patients }\end{array}$ & I & C & [168] \\
\hline $\begin{array}{l}\text { Supervised exercise training should be } \\
\text { considered in physically deconditioned } \\
\text { PAH patients under medical therapy }\end{array}$ & Ila & B & [153- \\
\hline $\begin{array}{l}\text { In-flight } \mathrm{O}_{2} \text { administration should } \\
\text { be considered for patients in WHO-FC III } \\
\text { and IV and those with arterial blood } \mathrm{O}_{2} \\
\text { pressure consistently <8 } \mathrm{kPa} \text { (60 } \mathrm{mmHg} \text { ) }\end{array}$ & Ila & $\mathrm{C}$ & \\
\hline $\begin{array}{c}\text { In elective surgery, epidural rather than } \\
\text { general anaesthesia should be preferred } \\
\text { whenever possible }\end{array}$ & Ila & $\mathrm{C}$ & \\
\hline $\begin{array}{c}\text { Excessive physical activity that leads to } \\
\text { distressing symptoms is not } \\
\text { recommended in PAH patients }\end{array}$ & III & $\mathrm{C}$ & \\
\hline
\end{tabular}

$\mathrm{O}_{2}$ : oxygen; $\mathrm{PAH}$ : pulmonary arterial hypertension; WHO-FC: World Health Organization functional class. ${ }^{\mathrm{a}}$ Class of recommendation.

${ }^{b}$ Level of evidence. ${ }^{c}$ Reference(s) supporting recommendations.

prescription of drugs, but is characterised by a complex strategy that includes the initial evaluation of severity and the subsequent response to treatment.

The current treatment strategy for PAH patients can be divided into three main steps [149]:

1. The initial approach includes general measures (physical activity and supervised rehabilitation, pregnancy, birth control and post-menopausal hormonal therapy, elective surgery, infection prevention, psychosocial support, adherence to treatments, genetic counselling and travel), supportive therapy (oral anticoagulants, diuretics, $\mathrm{O}_{2}$, digoxin), referral to expert centres and acute vasoreactivity testing for the indication of chronic CCB therapy.

2. The second step includes initial therapy with high-dose CCB in vasoreactive patients or drugs approved for $\mathrm{PAH}$ in non-vasoreactive patients according to the prognostic risk (Table 13) of the patient and the grade of recommendation and level of evidence for each individual compound or combination of compounds.

3. The third part is related to the response to the initial treatment strategy; in the case of an inadequate response, the role of combinations of approved drugs and lung transplantation are proposed.

\subsubsection{General measures}

Patients with PAH require sensible advice about general activities of daily living and need to adapt to the uncertainty associated with a serious chronic life-threatening disease. The diagnosis usually confers a degree of social isolation [150]. Encouraging patients and their family members to join patient support groups can have positive effects on coping, confidence and outlook. The recommendations for general measures are reported in the Table 16.

\subsubsection{Physical activity and supervised rehabilitation}

The 2009 PH guidelines suggested that PAH patients should be encouraged to be active within symptom limits [151]. It was recommended that patients should avoid excessive physical activity that leads to distressing symptoms, but when physically deconditioned, patients may undertake supervised exercise rehabilitation. This was based on a randomized controlled trial (RCT) that demonstrated an improvement 
in exercise and functional capacity and in quality of life in patients with $\mathrm{PH}$ who took part in a training programme as compared with an untrained control group [152]. Since then, additional uncontrolled experiences have supported these data utilising different models of exercise training [153-157]. Two additional RCTs have been published reporting that trained PAH patients reached higher levels of physical activity, had decreased fatigue severity and showed improved 6MWD, cardiorespiratory function and patient-reported quality of life as compared with untrained controls [158, 159]. The sample sizes of all these studies are quite small (ranging from 19 to 183 patients) and all or the initial training was highly supervised and in some instances conducted in an inpatient setting.

This recommendation is limited by gaps in the knowledge about the optimal method of exercise rehabilitation and the intensity and duration of the training. In addition, the characteristics of the supervision and the mechanisms for the improvement of symptoms, exercise and functional capacity are unclear, as are the possible effects on prognosis. Exercise training programmes should be implemented by centres experienced in both PAH patient care and rehabilitation of compromised patients. In addition, patients should be treated with the best standard of pharmacological treatment and in stable clinical condition before embarking on a supervised rehabilitation programme.

\subsubsection{Pregnancy, birth control and post-menopausal hormonal therapy}

Pregnancy remains associated with a substantial mortality rate in PAH. However, a recent report indicates that the outcome of pregnancies in PAH has improved, at least when PAH is well controlled, and particularly in long-term responders to CCBs [160]. During a 3-year period, the 13 participating centres reported 26 pregnancies. Three women (12\%) died and one (4\%) developed right heart failure requiring urgent heart-lung transplantation. There were eight abortions; two spontaneous and six induced. Sixteen pregnancies $(62 \%)$ were successful, i.e. the women delivered healthy babies without complications. A study from the USA from five centres between 1999 and 2009 managed 18 pregnancies with three deaths (17\%) [161]. These data must be confirmed by larger series before the general recommendation to avoid pregnancy in all patients with $\mathrm{PAH}$ is reconsidered. There is less consensus relating to the most appropriate methods of birth control. Barrier contraceptive methods are safe for the patient, but with an unpredictable effect. Progesterone-only preparations such as medroxyprogesterone acetate and etonogestrel are effective approaches to contraception and avoid the potential issues of oestrogens such as those associated with the old-generation mini-pill [162]. It should be remembered that the ERA bosentan may reduce the efficacy of oral contraceptive agents. The levonorgestrel-releasing intrauterine coil is also effective but may rarely lead to a vasovagal reaction when inserted, which may be poorly tolerated in severe PAH [162]. A combination of two methods may also be utilised. The patient who becomes pregnant should be informed of the high risk of pregnancy and termination of the pregnancy should be discussed. Those patients who choose to continue pregnancy should be treated with disease-targeted therapies, planned elective delivery and effective close collaboration between obstetricians and the PAH team $[163,164]$.

It is unclear whether the use of hormonal therapy in post-menopausal women with PAH is advisable. It may be considered in cases of intolerable menopausal symptoms in conjunction with oral anticoagulation.

\subsubsection{Elective surgery}

Elective surgery is expected to have an increased risk in patients with PAH. It is unclear as to which form of anaesthesia is preferable, but epidural is probably better tolerated than general anaesthesia [165-167]. Patients usually maintained on oral therapy may require temporary conversion to i.v. or nebulized treatment until they are able to both swallow and absorb drugs taken orally.

\subsubsection{Infection prevention}

Patients with PAH are susceptible to developing pneumonia, which is the cause of death in $7 \%$ of cases [34]. While there are no controlled trials, it is recommended to vaccinate against influenza and pneumococcal pneumonia.

\subsubsection{Psychosocial support}

$\mathrm{PH}$ is a disease with a significant impact on the psychological, social (including financial), emotional and spiritual functioning of patients and their families [168]. Teams managing these patients should have the skills and expertise to assess and manage issues in all of these domains, with close links to colleagues in relevant disciplines for those with severe problems, e.g. psychiatry, clinical psychology, welfare and social work. Patient support groups may also play an important role and patients should be advised to join such groups.

PH is a disease that may be severely life-limiting. In addition to psychological and social support there should be proactive advanced care planning with referral to specialist palliative care services when appropriate. 


\subsubsection{Adherence to treatments}

Adherence to medical treatments needs to be checked periodically due to the complexity of the PAH therapy and possible reductions or changes to the treatment regimen induced spontaneously by patients or non-expert physicians.

\subsubsection{Travel}

There are no studies using flight simulation to determine the need for supplemental $\mathrm{O}_{2}$ during prolonged flights in patients with PAH. The known physiological effects of hypoxia suggest that in-flight $\mathrm{O}_{2}$ administration should be considered for patients in WHO-FC III and IV and those with arterial blood $\mathrm{O}_{2}$ pressure consistently $<8 \mathrm{kPa}(60 \mathrm{mmHg})$ [169]. A flow rate of $2 \mathrm{l} / \mathrm{min}$ will raise inspired $\mathrm{O}_{2}$ pressure to values seen at sea level. Similarly, such patients should avoid going to altitudes $>1500-2000 \mathrm{~m}$ without supplemental $\mathrm{O}_{2}$. Patients should be advised to travel with written information about their PAH and be advised on how to contact local $\mathrm{PH}$ clinics in close proximity to where they are travelling.

\subsubsection{Genetic counselling}

Genetic counselling should be offered to selected PAH patients (detailed in section 5.1.12) [26]. Because of the psychological impact of the positive or negative results, genetic testing and counselling should be provided according to local regulations in the setting of a multidisciplinary team with availability of $\mathrm{PH}$ specialists, genetic counsellors, geneticists, psychologists and nurses. Affected individuals and at-risk family members may want to know their mutation status for family planning purposes. Current reproductive options for couples with a BMPR2 mutation carrier are to remain childless, to have no genetic prenatal testing (reproductive chance), to undergo prenatal or pre-implantation genetic diagnosis [170], to use gamete donation or to adopt.

\subsubsection{Supportive therapy}

The recommendations for supportive therapy are reported in Table 17.

\section{TABLE 17 Recommendations for supportive therapy}

\begin{tabular}{|c|c|c|c|}
\hline Recommendations & Class $^{a}$ & Levelb & Ref.c \\
\hline $\begin{array}{l}\text { Diuretic treatment is recommended in } \\
\text { PAH patients with signs of RV failure and } \\
\text { fluid retention }\end{array}$ & I & C & [178] \\
\hline $\begin{array}{l}\text { Continuous long-term } \mathrm{O}_{2} \text { therapy is } \\
\text { recommended in PAH patients when } \\
\text { arterial blood } \mathrm{O}_{2} \text { pressure is consistently } \\
<8 \mathrm{kPa}(60 \mathrm{mmHg}) \text { d }\end{array}$ & 1 & C & [179] \\
\hline $\begin{array}{l}\text { Oral anticoagulant treatment may be } \\
\text { considered in patients with IPAH, HPAH } \\
\text { and PAH due to use of anorexigens }\end{array}$ & Ilb & C & $\begin{array}{l}{[84,171} \\
175-177]\end{array}$ \\
\hline $\begin{array}{l}\text { Correction of anaemia and/or iron status } \\
\text { may be considered in PAH patients }\end{array}$ & IIb & C & [184] \\
\hline $\begin{array}{l}\text { The use of angiotensin-converting } \\
\text { enzyme inhibitors, angiotensin-2 } \\
\text { receptor antagonists, beta-blockers and } \\
\text { ivabradine is not recommended in } \\
\text { patients with PAH unless required by } \\
\text { co-morbidities (i.e. high blood pressure, } \\
\text { coronary artery disease or left heart } \\
\text { failure) }\end{array}$ & III & C & \\
\hline
\end{tabular}

HPAH: heritable pulmonary arterial hypertension; IPAH: idiopathic pulmonary arterial hypertension; $\mathrm{O}_{2}$ : oxygen; $\mathrm{PAH}$ : pulmonary arterial hypertension; RV: right ventricular. ${ }^{\mathrm{a}}$ Class of recommendation. ${ }^{\mathrm{b}}$ Level of evidence. ${ }^{\mathrm{c}}$ Reference(s) supporting recommendations. ${ }^{\mathrm{d}}$ See also recommendations for PAH associated with congenital cardiac shunts. 


\subsubsection{Oral anticoagulants}

There is a high prevalence of vascular thrombotic lesions at post-mortem examination in patients with IPAH [171]. Abnormalities in coagulation and fibrinolytic pathways have also been reported [172-174]. This, together with the non-specific increased risk factors for venous thromboembolism, including heart failure and immobility, represents the rationale for oral anticoagulation in PAH. Evidence in favour of oral anticoagulation is confined to patients with IPAH, HPAH and PAH due to anorexigens and is generally retrospective and based on single-centre experience [84, 171]. Registry and RCT data appear to be heterogeneous and inconclusive [175-177]. The potential benefits of oral anticoagulation in APAH is even less clear. Generally patients with PAH receiving therapy with long-term i.v. prostaglandins are anticoagulated in the absence of contraindications due in part to the additional risk of catheter-associated thrombosis. The role of the new oral anticoagulants in PAH is unknown. Additional information on APAH is provided in the individual chapters.

\subsubsection{Diuretics}

Decompensated right heart failure leads to fluid retention, raised central venous pressure, hepatic congestion, ascites and peripheral oedema. Although there are no RCTs on the use of diuretics in PAH, clinical experience shows clear symptomatic benefit in fluid overloaded patients treated with this therapy. The choice and dose of diuretic therapy may be left to the PAH physician [178]. The addition of aldosterone antagonists should also be considered together with systematic assessments of electrolyte plasma levels. It is important with diuretic use to monitor renal function and blood biochemistry in patients to avoid hypokalaemia and the effects of decreased intravascular volume leading to pre-renal failure.

\subsubsection{Oxygen}

Although $\mathrm{O}_{2}$ administration has been demonstrated to reduce the PVR in patients with PAH, there are no randomised data to suggest that long-term $\mathrm{O}_{2}$ therapy is beneficial. Most patients with $\mathrm{PAH}$, except those with CHD and pulmonary-to-systemic shunts, have minor degrees of arterial hypoxaemia at rest unless they have a patent foramen ovale. There are data showing that nocturnal $\mathrm{O}_{2}$ therapy does not modify the natural history of advanced Eisenmenger syndrome [179]. Guidance may be based on evidence in patients with COPD; when arterial blood $\mathrm{O}_{2}$ pressure is consistently $<8 \mathrm{kPa}(60 \mathrm{mmHg}$; alternatively, $<91 \%$ of arterial $\mathrm{O}_{2}$ saturation) patients are advised to take $\mathrm{O}_{2}$ to achieve an arterial blood $\mathrm{O}_{2}$ pressure $>8 \mathrm{kPa}$ [169]. Ambulatory $\mathrm{O}_{2}$ may be considered when there is evidence of symptomatic benefit and correctable desaturation on exercise.

\subsubsection{Digoxin and other cardiovascular drugs}

Digoxin has been shown to improve CO acutely in IPAH, although its efficacy is unknown when administered chronically [180]. It may be given to slow ventricular rate in patients with PAH who develop atrial tachyarrhythmias.

No convincing data are available on the usefulness and safety of angiotensin-converting enzyme inhibitors, angiotensin II receptor antagonists, beta-blockers or ivabradine in patients with $\mathrm{PAH}$.

\subsubsection{Anaemia and iron status}

Iron deficiency is common in patients with PAH and has been reported in $43 \%$ of patients with IPAH, $46 \%$ of patients with SSc-PAH and $56 \%$ of patients with Eisenmenger syndrome [181-183]. In all of these entities, preliminary data indicate that iron deficiency may be associated with reduced exercise capacity, and perhaps also with a higher mortality, independent of the presence or severity of anaemia [181, 182, $184,185]$. Based on these data, regular monitoring of the iron status should be considered in patients with $\mathrm{PAH}$ and detection of an iron deficiency should trigger a search for potential reasons. Iron substitution should be considered in patients with iron deficiency. Some studies suggest that oral iron absorption is impaired in patients with PAH, so i.v. iron administration may be preferable [181, 184, 186]. However, controlled trials are lacking.

\subsubsection{Specific drug therapy}

\subsubsection{Calcium channel blockers}

It has been increasingly recognised that only a small number of patients with IPAH who demonstrate a favourable response to acute vasodilator testing (Table 11) at the time of RHC do well with CCBs [84, 85]. The CCBs that have been predominantly used in reported studies are nifedipine, diltiazem and amlodipine, with particular emphasis on nifedipine and diltiazem $[84,85]$. The choice of CCB is based on the patient's heart rate at baseline, with a relative bradycardia favouring nifedipine and amlodipine and a relative tachycardia favouring diltiazem. The daily doses of these drugs that have shown efficacy in IPAH are relatively high: $120-240 \mathrm{mg}$ for nifedipine, $240-720 \mathrm{mg}$ for diltiazem and up to $20 \mathrm{mg}$ for amlodipine. 
It is advisable to start with an initial lower dose, e.g. $30 \mathrm{mg}$ of slow release nifedipine twice a day or $60 \mathrm{mg}$ of diltiazem three times a day (t.i.d.) or $2.5 \mathrm{mg}$ of amlodipine once a day, and increase cautiously and progressively to the maximum tolerated dose. Limiting factors for dose increase are usually systemic hypotension and lower limb peripheral oedema. Patients with IPAH who meet the criteria for a positive vasodilator response and are treated with CCBs should be followed closely for reasons of both safety and efficacy, with a complete reassessment after 3-4 months of therapy including RHC.

If the patient does not show an adequate response, defined as being in WHO-FC I or II and with a marked haemodynamic improvement (near normalization), additional PAH therapy should be instituted. In some cases the combination of $\mathrm{CCB}$ with the approved $\mathrm{PAH}$ drugs is required because of further clinical deterioration in case of CCB withdrawal attempts. Patients who have not undergone a vasoreactivity study or those with a negative study should not be started on CCBs because of potential severe side effects (e.g. hypotension, syncope and RV failure) [187].

Vasodilator responsiveness does not appear to predict a favourable long-term response to CCB therapy in patients with PAH in the setting of CTD, HIV, porto-pulmonary hypertension (PoPH) and PVOD [188, 189].

The recommendations for CCB therapy are reported in Table 18. For specific approved doses of the drugs, please refer to the updated official prescription information.

\subsubsection{Endothelin receptor antagonists}

Activation of the endothelin system has been demonstrated in both plasma and lung tissue of PAH patients [190]. Although it is unclear if the increases in endothelin-1 plasma levels are a cause or a consequence of $\mathrm{PH}$ [191], these data support a prominent role for the endothelin system in the pathogenesis of PAH [192]. Endothelin-1 exerts vasoconstrictor and mitogenic effects by binding to two distinct receptor isoforms in the

TABLE 18 Recommendations for calcium channel blocker therapy in patients who respond to the acute vasoreactivity test

\begin{tabular}{|c|c|c|c|}
\hline Recommendations & Class $^{a}$ & Levelb & Ref.c $^{\circ}$ \\
\hline $\begin{array}{l}\text { High doses of CCBs are recommended in } \\
\text { patients with IPAH, HPAH and DPAH who } \\
\text { are responders to acute vasoreactivity } \\
\text { testing }\end{array}$ & 1 & C & {$[84,85]$} \\
\hline $\begin{array}{l}\text { Close follow-up with complete } \\
\text { reassessment after 3-4 months of } \\
\text { therapy (including RHC) is } \\
\text { recommended in patients with IPAH, } \\
\text { HPAH and DPAH treated by high doses } \\
\text { of CCBs }\end{array}$ & I & C & {$[84,85]$} \\
\hline $\begin{array}{l}\text { Continuation of high doses of CCBs is } \\
\text { recommended in patients with IPAH, } \\
\text { HPAH and DPAH in WHO-FC I or II with } \\
\text { marked haemodynamic improvement } \\
\text { (near normalization) }\end{array}$ & I & C & {$[84,85]$} \\
\hline $\begin{array}{l}\text { Initiation of specific PAH therapy is } \\
\text { recommended in patients in WHO-FC III } \\
\text { or IV or those without marked } \\
\text { haemodynamic improvement (near } \\
\text { normalization) after high doses of CCBs }\end{array}$ & I & $C$ & {$[84,85]$} \\
\hline $\begin{array}{l}\text { High doses of CCBs are not indicated in } \\
\text { patients without a vasoreactivity study or } \\
\text { non-responders unless standard doses } \\
\text { are prescribed for other indications (e.g. } \\
\text { Raynaud's phenomenon) }\end{array}$ & III & $C$ & \\
\hline
\end{tabular}

CCB: calcium channel blocker; DPAH: drug-induced PAH; HPAH: heritable $\mathrm{PAH}$; IPAH: idiopathic PAH; PAH: pulmonary arterial hypertension; RHC: right heart catheterization; RV: right ventricular; WHO-FC: World Health Organization functional class. 
pulmonary vascular smooth muscle cells, endothelin receptors type A and B. The characteristics of RCTs with PAH drugs interfering with the endothelin pathway are reported in Web Table VIA.

\section{Ambrisentan}

Ambrisentan is an ERA that preferentially binds with endothelin receptor type A. Ambrisentan has been evaluated in a pilot study [193] and in two large RCTs that have demonstrated efficacy on symptoms, exercise capacity, haemodynamics and time to clinical worsening of patients with IPAH and PAH associated with CTD and HIV infection [194]. The incidence of abnormal liver function tests ranges from 0.8 to $3 \%$. Monthly liver function assessment is not mandated in the USA [195]. An increased incidence of peripheral oedema has been reported with ambrisentan use.

\section{Bosentan}

Bosentan is an oral active dual endothelin receptor type A and B antagonist and the first molecule of its class to be synthesized. Bosentan has been evaluated in PAH (idiopathic, associated with CTD and Eisenmenger syndrome) in six RCTs (Study-351, BREATHE-1, BREATHE-2, BREATHE-5, EARLY and COMPASS 2), which showed improvement in exercise capacity, FC, haemodynamics, echocardiographic and Doppler variables and time to clinical worsening [196-200]. Increases in hepatic aminotransferases occurred in approximately $10 \%$ of the patients and were found to be dose dependent and reversible after dose reduction or discontinuation. For these reasons, liver function testing should be performed monthly in patients receiving bosentan.

\section{Macitentan}

The dual ERA macitentan has been evaluated in an event-driven RCT [201]: 742 PAH patients were treated with $3 \mathrm{mg}$ or $10 \mathrm{mg}$ macitentan as compared with placebo for an average of 100 weeks. The primary endpoint was the time from the initiation of treatment to the first occurrence of a composite endpoint of death, atrial septostomy, lung transplantation, initiation of treatment with i.v. or subcutaneous prostanoids or worsening of PAH. Macitentan significantly reduced this composite endpoint of morbidity and mortality among patients with $\mathrm{PAH}$ and also increased exercise capacity. Benefits were shown both for patients who had not received treatment previously and for those receiving additional therapy for PAH. While no liver toxicity was shown, reduction in blood haemoglobin $\leqslant 8 \mathrm{~g} / \mathrm{dl}$ was observed in $4.3 \%$ of patients receiving $10 \mathrm{mg}$ of macitentan.

\subsubsection{Phosphodiesterase type 5 inhibitors and guanylate cyclase stimulators}

Inhibition of the cyclic guanosine monophosphate (cGMP) degrading enzyme phosphodiesterase type 5 results in vasodilation through the NO/cGMP pathway at sites expressing this enzyme. Since the pulmonary vasculature contains substantial amounts of phosphodiesterase type 5 , the potential clinical benefit of phosphodiesterase type 5 inhibitors (PDE-5is) has been investigated in PAH. In addition, PDE-5is exert antiproliferative effects [202, 203]. All three PDE-5is approved for the treatment of erectile dysfunction-sildenafil, tadalafil and vardenafil-cause significant pulmonary vasodilation, with maximum effects observed after 60, 75-90 and 40-45 minutes, respectively [204]. The characteristics of RCTs with $\mathrm{PAH}$ drugs interfering with the NO pathway [soluble guanylate cyclase (sGC) stimulators, PDE-5is] are reported in Web Table VIB.

\section{Sildenafil}

Sildenafil is an orally active, potent and selective inhibitor of phosphodiesterase type 5. Four RCTs in PAH patients treated with sildenafil have confirmed favourable results on exercise capacity, symptoms and/or haemodynamics [205-208]. An RCT addressing the effects of adding sildenafil to epoprostenol showed improvements after 12 weeks in 6MWD and time to clinical worsening. Of note, seven deaths occurred in this trial, all in the placebo group [209]. The approved dose of sildenafil is $20 \mathrm{mg}$ t.i.d. Most side effects of sildenafil are mild to moderate and mainly related to vasodilation (headache, flushing, epistaxis). Based on pharmacokinetic data, an i.v. formulation of sildenafil [210] has been proposed as a bridge for PAH patients on long-term oral treatment who are temporarily unable to ingest tablets.

\section{Tadalafil}

Tadalafil is a once-daily dispensed selective PDE-5i. An RCT in 406 PAH patients (53\% on background bosentan therapy) treated with tadalafil $2.5,10,20$ or $40 \mathrm{mg}$ once daily has shown favourable results on exercise capacity, symptoms, haemodynamics and time to clinical worsening at the highest dose [211]. The side-effect profile was similar to that of sildenafil. 
Vardenafil

Vardenafil is a twice-daily dispensed PDE-5i. An RCT in 66 treatment-naive PAH patients treated with vardenafil $5 \mathrm{mg}$ twice daily has shown favourable results on exercise capacity, haemodynamics and time to clinical worsening [212]. The side-effect profile was similar to that of sildenafil.

Riociguat

While PDE-5is such as sildenafil, tadalafil and vardenafil enhance the NO-cGMP pathway, slowing cGMP degradation, sGC stimulators enhance cGMP production [213]. Moreover, pre-clinical studies with sGC stimulators have antiproliferative and antiremodelling properties in various animal models.

An RCT [214] in 443 PAH patients (44\% and 6\% on background therapy with ERAs or prostanoids, respectively) treated with riociguat up to $2.5 \mathrm{mg}$ t.i.d. has shown favourable results on exercise capacity, haemodynamics, WHO-FC and time to clinical worsening. The increase in exercise capacity was also demonstrated in patients on background therapy. The most common serious adverse event in the placebo group and in the $2.5-\mathrm{mg}$ group was syncope ( $4 \%$ and $1 \%$, respectively). The combination of riociguat and PDE-5i is contraindicated due to hypotension and other relevant side effects detected in the open-label phase of an RCT study [215].

\subsubsection{Prostacyclin analogues and prostacyclin receptor agonists}

Prostacyclin is produced predominantly by endothelial cells and induces potent vasodilation of all vascular beds. This compound is the most potent endogenous inhibitor of platelet aggregation and also appears to have both cytoprotective and antiproliferative activities [216]. Dysregulation of the prostacyclin metabolic pathways has been shown in patients with $\mathrm{PAH}$ as assessed by a reduction of prostacyclin synthase expression in the pulmonary arteries and of prostacyclin urinary metabolites [217]. The clinical use of prostacyclin in patients with $\mathrm{PAH}$ has been extended by the synthesis of stable analogues that possess different pharmacokinetic properties but share qualitatively similar pharmacodynamic effects.

The characteristics of RCTs with PAH drugs interfering with the prostacyclin pathway (prostanoids and prostacyclin IP receptor agonists) are reported in Web Table VIC.

\section{Beraprost}

Beraprost is the first chemically stable and orally active prostacyclin analogue. An RCT [218] in Europe and a second in the USA [219] have shown an improvement in exercise capacity that persists up to 3-6 months. There were no haemodynamic improvements or long-term outcome benefits. The most frequent adverse events were headache, flushing, jaw pain and diarrhoea.

\section{Epoprostenol}

Epoprostenol (synthetic prostacyclin) has a short half-life (3-5 minutes) and is stable at room temperature for only 8 hours; it requires cooling and continuous administration by means of an infusion pump and a permanent tunnelled catheter. The efficacy of continuous i.v. administration of epoprostenol has been tested in three unblinded RCTs in patients with WHO-FC III and IV IPAH $[220,221]$ and in those with $\mathrm{PAH}$ associated with the scleroderma spectrum of diseases [222]. Epoprostenol improves symptoms, exercise capacity and haemodynamics in both clinical conditions and is the only treatment shown to reduce mortality in IPAH in a single RCT study [221]. The meta-analysis for total mortality of the three epoprostenol RCTs [220-222] has shown a risk reduction for mortality of about $70 \%$. Long-term persistence of efficacy has also been shown $[96,107]$ in IPAH as well as in other APAH conditions [223-225] and in non-operable CTEPH [226].

Treatment with epoprostenol is initiated at a dose of $2-4 \mathrm{ng} / \mathrm{kg} / \mathrm{min}$, with doses increasing at a rate limited by side effects (flushing, headache, diarrhoea, leg pain). The optimal dose varies between individual patients, ranging in the majority between 20 and $40 \mathrm{ng} / \mathrm{kg} / \mathrm{min}[96,107]$.

Serious adverse events related to the delivery system include pump malfunction, local site infection, catheter obstruction and sepsis. Guidelines for the prevention of central venous catheter bloodstream infections have been proposed [227]. Abrupt interruption of the epoprostenol infusion should be avoided, because in some patients this may lead to a PH rebound with symptomatic deterioration and even death. A thermostable formulation of epoprostenol is also available and does not usually require cooling packs to maintain stability beyond $8-12$ hours [228].

Iloprost

Iloprost is a chemically stable prostacyclin analogue available for i.v., oral or aerosol administration. Inhaled iloprost has been evaluated in one RCT in which daily repetitive iloprost inhalations (six to nine times, $2.5-5 \mu \mathrm{g}$ /inhalation, median $30 \mu \mathrm{g}$ daily) were compared with placebo inhalation in patients with 
PAH and CTEPH [229]. The study showed an increase in exercise capacity and improvement in symptoms, PVR and clinical events in enrolled patients. A second RCT involving 60 patients already treated with bosentan showed an increase in exercise capacity $(P<0.051)$ in the subjects randomized to the addition of inhaled iloprost compared with placebo [230]. Another similar study was terminated prematurely for futility [231]. Overall, inhaled iloprost was well tolerated, with flushing and jaw pain being the most frequent side effects. Continuous i.v. administration of iloprost appeared to be as effective as epoprostenol in a small series of patients with PAH and CTEPH [232]. The effects of oral iloprost have not been assessed in PAH.

\section{Treprostinil}

Treprostinil is a tricyclic benzidine analogue of epoprostenol, with sufficient chemical stability to be administered at ambient temperature. These characteristics allow administration of the compound by i.v. and subcutaneous routes. The subcutaneous administration of treprostinil can be accomplished by a micro-infusion pump and a small subcutaneous catheter. The effects of treprostinil in PAH were assessed in an RCT and showed improvements in exercise capacity, haemodynamics and symptoms [233]. The greatest exercise improvement was observed in patients who were more compromised at baseline and in subjects who could tolerate the upper quartile dose $(>13.8 \mathrm{ng} / \mathrm{kg} / \mathrm{min})$. Infusion site pain was the most common adverse effect of treprostinil, leading to discontinuation of the treatment in $8 \%$ of cases on active drug and limiting dose increases in an additional proportion of patients [233]. Treatment with subcutaneous treprostinil is initiated at a dose of $1-2 \mathrm{ng} / \mathrm{kg} / \mathrm{min}$, with doses increasing at a rate limited by side effects (local site pain, flushing, headache). The optimal dose varies between individual patients, ranging in the majority between 20 and $80 \mathrm{ng} / \mathrm{kg} / \mathrm{min}$.

An RCT was performed with i.v. treprostinil in PAH patients, but the enrolment of this trial was closed because of safety considerations after 45 (36\%) of the planned 126 patients had been randomized [234]. The data generated from 31 (25\%) survivors after the randomized phase (23 active and 8 placebo) are not considered reliable. The dose of i.v. treprostinil is two to three times higher than the dose of i.v. epoprostenol $[235,236]$.

An RCT with inhaled treprostinil in PAH patients on background therapy with either bosentan or sildenafil showed improvements in the 6MWD by $20 \mathrm{~m}$ at peak dose and $12 \mathrm{~m}$ at trough dose, NT-proBNP and quality of life measures [237].

Oral treprostinil has been evaluated in two RCTs in PAH patients on background therapy with bosentan and/or sildenafil and in both trials the primary endpoint 6MWD did not reach statistical significance [238, 239]. An additional RCT in treatment-naive PAH patients showed improvement in the 6MWD by $26 \mathrm{~m}$ at peak dose and $17 \mathrm{~m}$ at trough dose [240].

\section{Selexipag}

Selexipag is an orally available, selective prostacyclin IP receptor agonist. Although selexipag and its metabolite have modes of action similar to that of endogenous prostacyclin (IP receptor agonism), they are chemically distinct from prostacyclin with a different pharmacology. In a pilot RCT in PAH patients (receiving stable ERA and/or PDE-5i therapy), selexipag reduced PVR after 17 weeks [241]. An event-driven phase 3 RCT that enrolled 1156 patients [248] has shown that selexipag alone or on top of mono- or double therapy with ERAs and/or PDE-5i was able to reduce by $40 \%$ (hazard ratio $0.60, P<$ 0.0001 ) a composite morbidity and mortality endpoint (including death from all causes, hospitalization for worsening of $\mathrm{PAH}$, worsening of $\mathrm{PAH}$ resulting in the need for lung transplantation or atrial septostomy, initiation of parenteral prostanoids or chronic $\mathrm{O}_{2}$ for worsening of $\mathrm{PAH}$ and disease progression).

Recommendations for efficacy of specific drug monotherapies are reported in the Table 19.

\subsubsection{Experimental compounds and strategies}

Despite progress in the treatment of $\mathrm{PAH}$, the functional limitation and survival of these patients remain unsatisfactory. There are three well-known pathways that contribute to the pathogenesis of PAH: the endothelin, NO and prostacyclin pathways. Treatments targeting these pathways are well established in clinical practice, such as ERAs, PDE-5is and prostanoids. Additional therapeutic strategies targeted at diverse pathobiological changes are being explored in order to further improve symptoms and prognosis. Three pathways have been explored with unsatisfactory results using the following compounds: inhaled vasoactive intestinal peptide, tyrosine kinase inhibitors (platelet-derived growth factor inhibitors) and serotonin antagonists. The following additional compounds are in an earlier stage of development: rho kinase inhibitors, vascular endothelial growth factor receptor inhibitors, angiopoietin-1 inhibitors and elastase inhibitors. Gene therapy strategies have been tested in animal models. Stem cell therapy has proven to be effective in the monocrotaline rat model and is currently being tested in a proof-of-concept 
TABLE 19 Recommendations for efficacy of drug monotherapy for pulmonary arterial hypertension (group 1) according to World Health Organization functional class. The sequence is by pharmacological group, by rating and by alphabetical order

\begin{tabular}{|c|c|c|c|c|c|c|c|c|c|}
\hline \multirow{2}{*}{\multicolumn{3}{|c|}{ Measure/treatment }} & \multicolumn{6}{|c|}{ Class ${ }^{\mathrm{a}}$-level ${ }^{\mathrm{b}}$} & \multirow{3}{*}{$\begin{array}{l}\text { Ref.c } \\
{[84,85]}\end{array}$} \\
\hline & & & \multicolumn{2}{|c|}{ WHO-FC II } & \multicolumn{2}{|c|}{ WHO-FC III } & \multicolumn{2}{|c|}{ WHO-FC IV } & \\
\hline \multicolumn{3}{|l|}{ Calcium channel blockers } & 1 & $C^{d}$ & I & $C^{d}$ & - & - & \\
\hline \multirow{3}{*}{$\begin{array}{l}\text { Endothelin receptor } \\
\text { antagonists }\end{array}$} & \multicolumn{2}{|l|}{ Ambrisentan } & I & A & 1 & A & Ilb & C & [194] \\
\hline & \multicolumn{2}{|l|}{ Bosentan } & 1 & A & 1 & A & IIb & C & [196-200] \\
\hline & \multicolumn{2}{|l|}{ Macitentane } & । & B & 1 & $\mathrm{~B}$ & $\mathrm{Ilb}$ & C & [201] \\
\hline \multirow{3}{*}{$\begin{array}{l}\text { Phosphodiesterase type } 5 \\
\text { inhibitors }\end{array}$} & \multicolumn{2}{|l|}{ Sildenafil } & । & A & I & A & IIb & C & [205-208] \\
\hline & \multicolumn{2}{|l|}{ Tadalafil } & 1 & $\mathrm{~B}$ & I & $\mathrm{B}$ & $\mathrm{IIb}$ & C & [211] \\
\hline & \multicolumn{2}{|l|}{ Vardenafilg } & $\mathrm{Ilb}$ & $\mathrm{B}$ & $\mathrm{IIb}$ & $\mathrm{B}$ & IIb & C & [212] \\
\hline $\begin{array}{l}\text { Guanylate cyclase } \\
\text { stimulators }\end{array}$ & \multicolumn{2}{|l|}{ Riociguat } & I & B & 1 & B & IIb & C & [214] \\
\hline \multirow[t]{8}{*}{ Prostacyclin analogues } & Epoprostenol & Intravenouse & - & - & I & A & I & A & [220-222] \\
\hline & \multirow[t]{2}{*}{ Iloprost } & Inhaled & - & - & 1 & $\mathrm{~B}$ & IIb & C & [229-231] \\
\hline & & Intravenousg & - & - & Ila & C & $\mathrm{Ilb}$ & C & [232] \\
\hline & \multirow[t]{4}{*}{ Treprostinil } & Subcutaneous & - & - & 1 & $\mathrm{~B}$ & $\mathrm{Ilb}$ & C & [233] \\
\hline & & Inhaledg & - & - & 1 & $\mathrm{~B}$ & $\mathrm{Ilb}$ & C & [237] \\
\hline & & Intravenous ${ }^{f}$ & - & - & Ila & C & IIb & C & [234] \\
\hline & & Oralg & - & - & $\mathrm{Ilb}$ & $\mathrm{B}$ & - & - & [238-240] \\
\hline & \multicolumn{2}{|l|}{ Beraprostg } & - & - & $\mathrm{Ilb}$ & $\mathrm{B}$ & - & - & [218] \\
\hline IP receptor agonists & \multicolumn{2}{|c|}{ Selexipag (oral)g } & 1 & $\mathrm{~B}$ & I & $\mathrm{B}$ & - & - & {$[241,248]$} \\
\hline
\end{tabular}

EMA: European Medicines Agency; PAH: pulmonary arterial hypertension; RCT: randomized controlled trial; WHO-FC: World Health Organization functional class. ${ }^{a}$ Class of recommendation. ${ }^{b}$ Level of evidence. ${ }^{\mathrm{C}}$ Reference(s) supporting recommendations. ${ }^{\mathrm{d}}$ Only in responders to acute vasoreactivity tests $=$ class I, for idiopathic PAH, heritable PAH and PAH due to drugs; class Ila, for conditions associated with PAH. ${ }^{~}$ Time to clinical worsening as primary endpoint in RCTs or drugs with demonstrated reduction in all-cause mortality. In patients not tolerating the subcutaneous form. ${ }^{9}$ This drug is not approved by the EMA at the time of publication of these guidelines.

and dose-finding study in PAH patients. A controversial study has shown a preliminary favourable effect of PA denervation by a radiofrequency ablation catheter [242, 243].

\subsubsection{Combination therapy}

Combination therapy-using two or more classes of drugs simultaneously-has been used successfully in the treatment of systemic hypertension and heart failure. It is also an attractive option for the management of PAH, because three separate signalling pathways known to be involved in the disease can be addressed by specific drugs: the prostacyclin pathway (prostanoids), the endothelin pathway (ERAs) and the NO pathway (PDE-5is and sGCs).

The experience with combination therapy is increasing and a recent meta-analysis on six RCTs with combination therapy including 858 patients has been published [244]; compared with the control group, combination therapy reduced the risk of clinical worsening \{relative risk [RR] 0.48 [95\% confidence interval (CI) $0.26,0.91], P=0.023\}$, increased the $6 \mathrm{MWD}$ significantly by $22 \mathrm{~m}$ and reduced mean PAP, RAP and PVR. The incidence of serious adverse events was similar in the two groups [RR 1.17 (95\% CI $0.40,3.42), P=0.77]$. The reduction in all-cause mortality was not statistically significant. However, the incidence of mortality in RCTs with PAH medications is relatively low and to achieve statistical significance a sample size of several thousand patients may be required [244]. 
Combination therapy may be applied sequentially or initially (upfront).

Sequential combination therapy is the most widely utilised strategy both in RCTs and in clinical practice: from monotherapy there is an addition of a second and then a third drug in cases of inadequate clinical results or in cases of deterioration. A structured prospective programme to evaluate the adequacy of clinical results is the so-called goal-oriented therapy, a treatment strategy that uses known prognostic indicators as treatment targets. The therapy is considered adequate only if the targets are met. The key difference between goal-oriented therapy and non-structured approaches is that patients who are stabilised, or even those who improve slightly, can still receive additional therapy if treatment goals are not met. The goal-oriented treatment strategy utilises different targets, including WHO-FC I or II, and the near-normalization of resting CI and/or of NT-proBNP plasma levels. A recent study has confirmed a better prognosis in patients achieving these goals as compared with patients who did not [97].

Recommendations and evidence on the use of specific drugs for initial combination therapy and for sequential combination therapy for PAH according to WHO-FC are provided in Table 20 and Table 21, respectively.

The rationale for initial or upfront combination therapy is based on the known mortality of PAH, which is reminiscent of many malignancies, and the fact that malignancies and critical medical illnesses (heart failure, malignant hypertension) are not treated with a stepwise approach to therapy, but rather with pre-emptive combination therapy. The experience in RCTs with initial combination therapy started with the small BREATHE-2 (Web Table VID) study, which failed to demonstrate any significant difference between patients treated initially with the combination epoprostenol and bosentan as compared with epoprostenol alone [198]. In a more recent experience, 23 treatment-naive PAH patients were treated with the initial combination of epoprostenol and bosentan and compared with a matched historical control group treated with epoprostenol [245]. The study showed a statistically significant greater decrease in PVR in the initial combination therapy group, but this haemodynamic benefit did not translate into a statistically significant

TABLE 20 Recommendations for efficacy of initial drug combination therapy for pulmonary arterial hypertension (group 1) according to World Health Organization functional class. Sequence is by rating

\begin{tabular}{|l|c|c|c|c|c|c|c|}
\hline \multirow{2}{*}{ Measure/treatment } & \multicolumn{5}{|c|}{ Class $^{\text {a-Levelb }}$} & \multirow{2}{*}{ Ref.c } \\
\cline { 2 - 7 } & WHO-FC II & WHO-FC III & WHO-FC IV & \\
\hline $\begin{array}{l}\text { Ambrisentan + } \\
\text { tadalafild }\end{array}$ & I & B & I & B & Ilb & C & [247] \\
\hline Other ERA + PDE-5i & Ila & C & Ila & C & Ilb & C & - \\
\hline $\begin{array}{l}\text { Bosentan + } \\
\text { sildenafil + } \\
\text { i.v. epoprostenol }\end{array}$ & - & - & Ila & C & Ila & C & {$[246]$} \\
\hline $\begin{array}{l}\text { Bosentan + i.v. } \\
\text { epoprostenol }\end{array}$ & - & - & Ila & C & Ila & C & {$[198,245]$} \\
\hline $\begin{array}{l}\text { Other ERA or } \\
\text { PDE-5i + } \\
\text { s.c. treprostinil }\end{array}$ & & & Ilb & C & Ilb & C & - \\
\hline $\begin{array}{l}\text { Other ERA or } \\
\text { PDE-5i + other } \\
\text { i.v. prostacyclin } \\
\text { analogues }\end{array}$ & & & & & & & \\
\hline
\end{tabular}

ERA: endothelin receptor antagonist; i.v.: intravenous; PDE-5i: phosphodiesterase type 5 inhibitor; RCT: randomized controlled trial; s.c.: subcutaneous; WHO-FC: World Health Organization functional class. ${ }^{a}$ Class of recommendation. ${ }^{b}$ Level of evidence. ${ }^{c}$ Referencels) supporting recommendations. 'Time to clinical failure as primary endpoint in RCTs or drugs with demonstrated reduction in all-cause mortality (prospectively defined). 
TABLE 21 Recommendations for efficacy of sequential drug combination therapy for pulmonary arterial hypertension (group 1) according to World Health Organization functional class. Sequence is by rating and by alphabetical order

\begin{tabular}{|c|c|c|c|c|c|c|c|}
\hline \multirow{3}{*}{\begin{tabular}{|l} 
Measure/treatment \\
$\begin{array}{l}\text { Macitentan added to } \\
\text { sildenafild }\end{array}$
\end{tabular}} & \multicolumn{6}{|c|}{ Class $^{a}$-Level ${ }^{b}$} & \multirow{3}{*}{$\begin{array}{l}\text { Ref.c }^{c} \\
{[201]}\end{array}$} \\
\hline & \multicolumn{2}{|c|}{ WHO-FC II } & \multicolumn{2}{|c|}{ WHO-FC III } & \multicolumn{2}{|c|}{ WHO-FC IV } & \\
\hline & I & $\mathrm{B}$ & 1 & B & Ila & C & \\
\hline $\begin{array}{l}\text { Riociguat added } \\
\text { to bosentan }\end{array}$ & I & $\mathrm{B}$ & I & B & Ila & C & [214] \\
\hline $\begin{array}{l}\text { Selexipage added to } \\
\text { ERA and/or PDE-5id }\end{array}$ & I & $\mathrm{B}$ & I & B & Ila & C & {$[241,248]$} \\
\hline $\begin{array}{l}\text { Sildenafil added } \\
\text { to epoprostenol }\end{array}$ & - & - & I & B & Ila & B & [209] \\
\hline $\begin{array}{l}\text { Treprostinil inhaled } \\
\text { added to sildenafil } \\
\text { or bosentan }\end{array}$ & Ila & $\mathrm{B}$ & Ila & B & Ila & C & [237] \\
\hline $\begin{array}{l}\text { Iloprost inhaled } \\
\text { added to bosentan }\end{array}$ & IIb & $\mathrm{B}$ & IIb & B & $\mathrm{IIb}$ & C & {$[230,231]$} \\
\hline $\begin{array}{l}\text { Tadalafil added } \\
\text { to bosentan }\end{array}$ & Ila & C & Ila & C & Ila & C & [211] \\
\hline $\begin{array}{l}\text { Ambrisentan added } \\
\text { to sildenafil }\end{array}$ & IIb & C & IIb & C & Ilb & C & [249] \\
\hline $\begin{array}{l}\text { Bosentan added to } \\
\text { epoprostenol }\end{array}$ & - & - & IIb & C & $\mathrm{Ilb}$ & C & [250] \\
\hline $\begin{array}{l}\text { Bosentan added to } \\
\text { sildenafil }\end{array}$ & IIb & C & IIb & C & $\mathrm{Ilb}$ & C & {$[251,252]$} \\
\hline $\begin{array}{l}\text { Sildenafil added } \\
\text { to bosentan }\end{array}$ & IIb & C & $\mathrm{IIb}$ & C & $\mathrm{Ilb}$ & C & [252] \\
\hline $\begin{array}{l}\text { Other double } \\
\text { combinations }\end{array}$ & IIb & C & IIb & C & IIb & C & - \\
\hline $\begin{array}{l}\text { Other triple } \\
\text { combinations }\end{array}$ & IIb & C & IIb & C & $\mathrm{Ilb}$ & C & - \\
\hline $\begin{array}{l}\text { Riociguat added to } \\
\text { sildenafil or other } \\
\text { PDE-5i }\end{array}$ & III & $\mathrm{B}$ & III & B & III & $\mathrm{B}$ & [215] \\
\hline
\end{tabular}

EMA: European Medicines Agency; ERA: endothelin receptor antagonist; PAH: pulmonary arterial hypertension; PDE-5i: phosphodiesterase type 5 inhibitor; RCT: randomized controlled trial; WHO-FC: World Health Organization functional class. ${ }^{\text {a Class of }}$ recommendation. ${ }^{\mathrm{b}}$ Level of evidence. ${ }^{\mathrm{c}}$ Reference(s) supporting recommendations. ${ }^{\mathrm{d}}$ Time to clinical worsening as primary endpoint in RCTs or drugs with demonstrated reduction in all-cause mortality (prospectively defined). ${ }^{\mathrm{e}}$ This drug was not approved by the EMA at the time of publication of these guidelines.

difference in survival or in transplant-free survival. A pilot study on an initial triple combination in 19 WHO-FC class III and IV patients has provided preliminary evidence of the long-term benefits of upfront triple combination therapy in patients with severe PAH [246]. A recent multicentre, multinational, blinded, placebo-controlled trial (Web Table VID) compared first-line monotherapy with tadalafil or monotherapy with ambrisentan with upfront combination therapy with tadalafil and ambrisentan in de novo WHO-FC II and III PAH patients [247]. The primary endpoint was a composite of clinical failure events (including 
death, hospitalization, PAH progression and unsatisfactory clinical status). The study was positive, with a $50 \%$ reduction in events in the combination group. In addition, improvements were observed in exercise capacity, rate of satisfactory clinical response and NT-proBNP plasma levels [247].

\subsubsection{Drug interactions}

Significant drug interactions involving the disease-targeted therapies for PAH are shown in Web Table VII. This table highlights known important interactions but does not include theoretical untested interactions, which may still be clinically important. In addition, updated official prescribing information for each compound should be read.

Bosentan is an inducer of cytochrome P450 isoenzymes CYP3A4 and CYP2C9. Plasma concentrations of drugs metabolised by these isoenzymes will be reduced when co-administered with bosentan. Bosentan is also metabolised by these enzymes so that their inhibition may increase the plasma concentration of bosentan. In addition to interactions shown in Web Table VII, a combination of a potent CYP3A4 inhibitor (ketoconazole, ritonavir) and/or a CYP2C9 inhibitor (e.g. amiodarone, fluconazole) with bosentan may cause a significant increase in plasma bosentan levels and thus is contraindicated. Interactions may theoretically occur with itraconazole, tacrolimus, sirolimus, carbamazepine, phenytoin, phenobarbitone, dapsone and St John's wort.

Sildenafil is metabolised by cytochrome P450 isoenzymes CYP3A4 (major route) and CYP2C9 (minor route). There is an increase in sildenafil bioavailability and reduced clearance with CYP3A4 substrates and inhibitors and CYP3A4 substrates plus beta-adrenoceptor blockers. CYP3A4 inducers such as carbamazepine, phenytoin, phenobarbital, rifampicin and St John's wort may significantly lower sildenafil levels. Sildenafil levels are modestly increased by fresh grapefruit juice, a weak inhibitor of CYP3A4.

Finally, care is needed when PAH-targeted medications are co-administered with antihypertensive drugs such as beta-adrenoceptor blockers, angiotensin-converting enzyme inhibitors, etc., to avoid excessive systemic hypotension.

\subsubsection{Balloon atrial septostomy}

The creation of an inter-atrial right-to-left shunt can decompress the right heart chambers and increase LV preload and $\mathrm{CO}[253,254]$. In addition, this improves systemic $\mathrm{O}_{2}$ transport despite arterial $\mathrm{O}_{2}$ desaturation [253] and decreases sympathetic hyperactivity. The recommended technique is graded balloon dilation atrial septostomy, which produces equivalent improvements in haemodynamics and symptoms but reduced risk compared with the original blade technique. Other techniques are considered experimental [255].

A careful pre-procedure risk assessment ensures reduced mortality. Balloon atrial septostomy (BAS) should be avoided in end-stage patients presenting with a baseline mean RAP $>20 \mathrm{mmHg}$ and $\mathrm{O}_{2}$ saturation at rest $<85 \%$ on room air. Patients should be on optimal medical therapy, which may include preconditioning with i.v. inotropic drugs, prior to considering BAS. Published reports suggest a benefit in patients who are in WHO-FC IV with right heart failure refractory to medical therapy or with severe syncopal symptoms $[253,254]$. It may also be considered in patients awaiting lung transplantation with unsatisfactory clinical response on maximal medical therapy or when medical therapy is not available. Studies show improvements in CI and decreases in RAP with improvement in 6MWD [253, 254]. The impact of BAS on long-term survival has not been established in RCTs [253, 254]. BAS should be regarded as a palliative or bridging procedure to be performed only in centres with experience in the method [256]. Since BAS is performed very rarely, it has not been included in the treatment algorithm (Figure 2).

\subsubsection{Advanced right ventricular failure}

6.3.7.1 Intensive care unit management

Patients with PH may require intensive care unit (ICU) treatment for a co-morbid condition (including major surgery), right heart failure or both. In a series from France, the mortality among PAH patients admitted to the ICU was $41 \%$ [257], underscoring the poor prognosis. Hence PAH patients requiring ICU treatment should be managed at specialized centres whenever possible. Basic monitoring includes vital signs (heart rate, blood pressure, body temperature and $\mathrm{O}_{2}$ saturation), urine production, central venous pressure, central venous $\mathrm{O}_{2}$ saturation and blood lactate levels. The combination of a low central venous $\mathrm{O}_{2}$ saturation $(<60 \%)$ with rising lactate levels and low or absent urine production signals imminent right heart failure. In certain situations, placement of a right heart catheter might be required to allow comprehensive haemodynamic monitoring. The basic principles of ICU management of patients with $\mathrm{PH}$ and RV failure include the treatment of triggering factors (such as anaemia, arrhythmias, infections or other co-morbidities), optimization of fluid balance (usually with i.v. diuretics), reduction of RV afterload (usually with parenteral prostacyclin analogues, but sometimes also with other PAH drugs), improvement of $\mathrm{CO}$ with inotropes (with dobutamine being the preferred inotrope to treat RV failure) and maintenance 


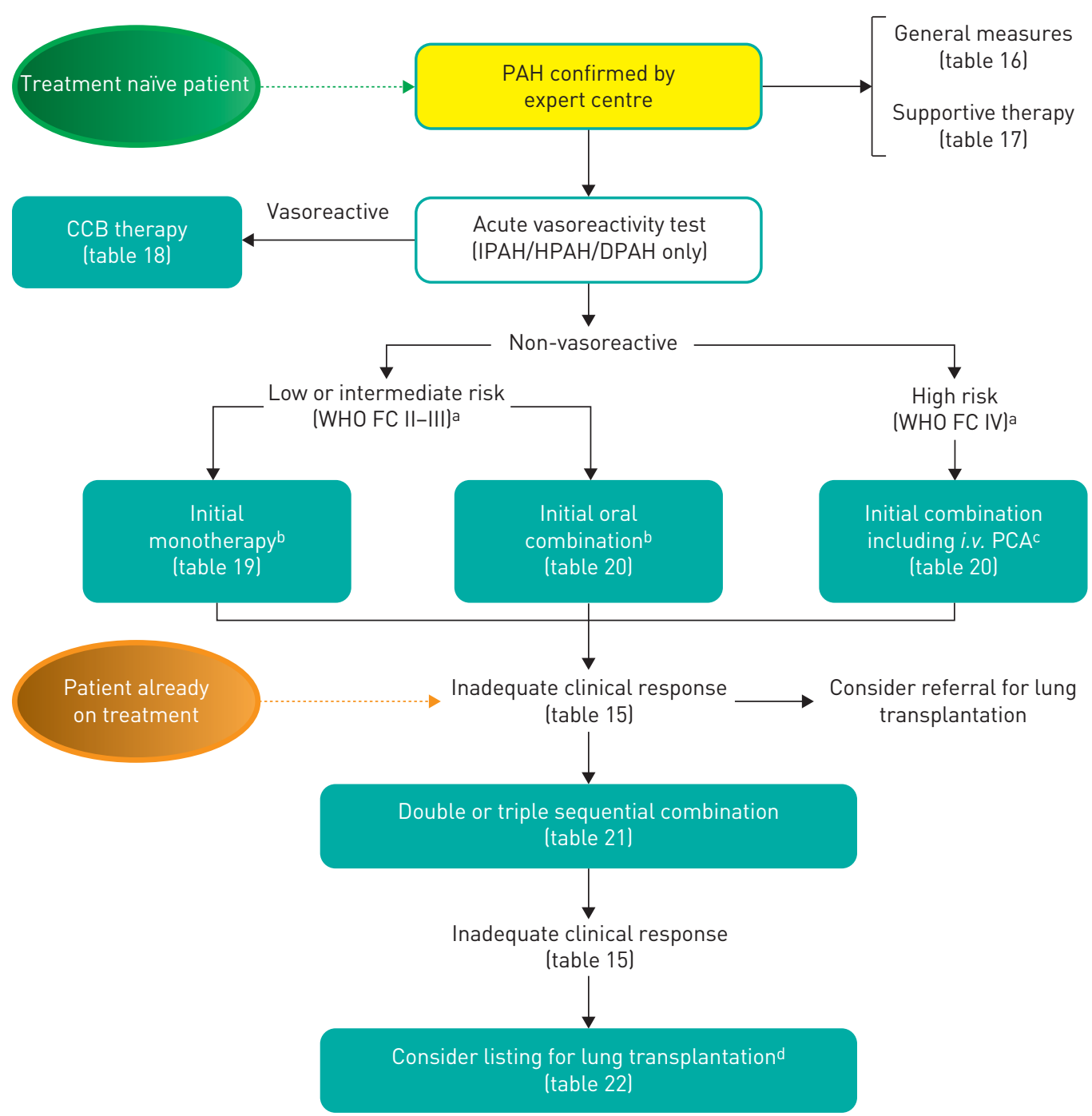

FIGURE 2 Evidence based treatment algorithm for pulmonary arterial hypertension patients (for group 1 patients only; see description in the text). CCB: calcium channel blockers; DPAH: drug-induced PAH; HPAH: heritable PAH; IPAH: idiopathic PAH; i.v.: intravenous; PAH: pulmonary arterial hypertension; PCA:

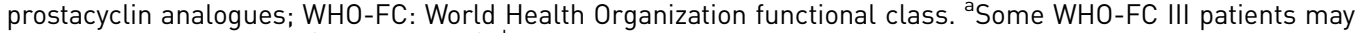
be considered high risk (see Table 13). ' Initial combination with ambrisentan plus tadalafil has proven to be superior to initial monotherapy with ambrisentan or tadalafil in delaying clinical failure. "Intravenous epoprostenol should be prioritised as it has reduced the 3 months rate for mortality in high risk PAH patients also as monotherapy. ${ }^{\mathrm{d}}$ Consider also balloon atrial septostomy.

of systemic blood pressure with vasopressors, if necessary [258-260]. Intubation should be avoided in patients with RV failure, as it frequently results in haemodynamic collapse.

\subsubsection{Right ventricle assistance}

The use of veno-arterial extracorporeal membrane oxygenation (ECMO) should be considered for selected patients with $\mathrm{PH}$ and RV failure. A veno-venous approach may improve oxygenation but does not unload the RV, which makes it unsuitable for this patient population. There are two basic concepts for the use of ECMO in these patients: bridge to recovery and bridge to transplantation. Few reports have been published on the bridge to recovery concept [261], which is only reasonable in patients for whom a clear therapeutic concept with a realistic chance of recovery exists. There are, however, several reports on the successful use of ECMO as a bridge to transplantation, especially when used in awake patients [261-263]. An alternative approach involves connecting a pumpless device to the pulmonary circulation [264, 265]. All of these procedures are available only in highly specialized centres.

\subsubsection{Transplantation}

The advent of disease-targeted therapy for severe $\mathrm{PAH}$ has reduced and delayed patient referral for lung transplant programmes [256]. The long-term outcomes of medically treated patients remain uncertain and 
transplantation should continue to be an important option for those who fail on such therapy and remain in WHO-FC III or IV $[96,107]$. Delayed referral in combination with the length of the waiting time, due to the shortage of organ donors, may increase the mortality of patients on the waiting list and their clinical severity at the time of transplantation.

The overall 5-year survival following transplantation for PAH was considered to be $45-50 \%$, with evidence of continued good quality of life [266]. More recent data show that survival is increased to $52-75 \%$ at 5 years and to $45-66 \%$ at 10 years [267-269].

Considering the above information, it seems reasonable to consider eligibility for lung transplantation after an inadequate clinical response to the initial monotherapy and to refer the patient soon after an inadequate clinical response is confirmed on maximal combination therapy (Figure 2). Also the aetiology of PAH may help the decision making since the prognosis varies according to the underlying condition. In fact, PAH associated with CTD has a worse prognosis than IPAH, even when treated with prostanoids, while patients with PAH associated with $\mathrm{CHD}$ have a better survival. The poorest prognosis is seen in patients with PVOD and PCH because of the lack of effective medical treatments; these patients should be listed for transplantation at diagnosis.

Both heart-lung and double-lung transplantation have been performed for $\mathrm{PAH}$, although the threshold for unrecoverable RV systolic dysfunction and/or LV diastolic dysfunction is unknown. Currently the vast majority of patients worldwide receive bilateral lungs, as indicated by the International Society for Heart and Lung Transplantation Registry figures [270]. Patients with Eisenmenger syndrome due to simple shunts have been treated with isolated lung transplantation and repair of the cardiac defect or by heartlung transplantation [266].

While registry data initially supported a survival benefit of heart-lung transplantation for patients with $\mathrm{PH}$ associated with a ventricular septal defect [271], experience with isolated bilateral lung transplantation has increased and more recent data support a role for this approach combined with repair of the defect [272].

Recent reports indicate that veno-arterial ECMO may be employed in awake end-stage PH patients for bridging to lung transplantation [263].

\subsubsection{Treatment algorithm}

A treatment algorithm for PAH patients is shown in Figure 2. The classes of recommendation and levels of evidence for the PAH treatments are shown in Tables 19 (monotherapy), 20 (initial combination therapy), 21 (sequential combination therapy) and 22 (ICU and interventions). Definitions of clinical response to treatments are reported in Table 15. It is recognized that the therapeutic approach to PAH may vary depending on the local availability (and expertise) of therapeutic options in various hospitals and clinical settings. Accordingly, Tables 19, 20, 21 and 22 provide the necessary evidence for alternative evidence-based therapeutic strategies. In these tables only the compounds officially approved for $\mathrm{PAH}$ or undergoing

TABLE 22 Recommendations for efficacy of intensive care unit management, balloon atrial septostomy and lung transplantation for pulmonary arterial hypertension (group 1) according to World Health Organization functional class

\begin{tabular}{|c|c|c|c|c|c|c|c|}
\hline \multirow{3}{*}{$\begin{array}{l}\text { Measure/treatment } \\
\text { Hospitalization in ICU is recommended in } \mathrm{PH} \text { patients with } \\
\text { high heart rate ( }>110 \text { beats } / \mathrm{min} \text { ), low blood pressure } \\
\text { (systolic blood pressure }<90 \mathrm{mmHg} \text { ), low urine output and } \\
\text { rising lactate levels due or not due to co-morbidities }\end{array}$} & \multicolumn{6}{|c|}{ Class $^{a}-$ Level $^{b}$} & \multirow{3}{*}{$\begin{array}{l}\text { Ref.c } \\
{[257]}\end{array}$} \\
\hline & \multicolumn{2}{|c|}{ WHO-FC II } & \multicolumn{2}{|c|}{ WHO-FC III } & \multicolumn{2}{|c|}{ WHO-FC IV } & \\
\hline & - & - & - & - & 1 & C & \\
\hline Inotropic support is recommended in hypotensive patients & & & । & C & 1 & C & \\
\hline $\begin{array}{l}\text { Lung transplantation is recommended soon after } \\
\text { inadequate clinical response on maximal medical therapy }\end{array}$ & - & - & I & C & 1 & C & [270] \\
\hline $\begin{array}{l}\text { BAS may be considered where available after failure of } \\
\text { maximal medical therapy }\end{array}$ & - & - & IIb & C & Ilb & C & {$[253,254]$} \\
\hline
\end{tabular}

BAS: Balloon atrial septostomy; ICU: intensive care unit; $\mathrm{PH}$ : pulmonary hypertension; WHO-FC: World Health Organization functional class. ${ }^{a}$ Class of recommendation. ${ }^{b}$ Level of evidence. ${ }^{c}$ Reference(s) supporting recommendations. 
regulatory approval in at least one country are included. A four-level hierarchy for endpoints in RCTs has been proposed by experts according to the level of evidence regarding efficacy [273, 274]. According to this hierarchy, drugs or combinations of drugs with time to clinical failure or to clinical worsening as the primary endpoint in RCTs or drugs with a demonstrated reduction in all-cause mortality (prospectively defined) have been highlighted with a footnote in Tables 19, 20 and 21. The PAH treatment algorithm does not apply to patients in other clinical groups, and in particular it does not apply to patients with $\mathrm{PH}$ associated with group 2 (LHD) or group 3 (lung diseases). In addition, the different treatments have been evaluated by RCTs mainly in IPAH, HPAH, PAH due to drugs and in PAH associated with CTD or with CHD (surgically corrected or not).

Treatment algorithm description (Figure 2)

- After confirmation of the diagnosis of the treatment-naive PAH patient in an expert centre, the suggested initial approach is the adoption of general measures and the initiation of supportive therapy if needed (Tables 16 and 17).

- Acute vasoreactivity testing should be performed only in patients with IPAH, HPAH and PAH associated with drugs or toxins use. Vasoreactive patients should be treated with high doses (progressively titrated) of CCB; adequate response should be confirmed after 3-4 months of treatment (Table 18). Patient responders without an adequate clinical response to CCB treatment should be treated with approved PAH medications according to the non-vasoreactive patient's treatment strategy.

- Non-responders to acute vasoreactivity testing who are at low or intermediate risk (Table 13) can be treated with either initial monotherapy (Table 19) or initial oral combination therapy (Table 20).

- If initial monotherapy is chosen, since head-to-head comparisons among different compounds are not available, no evidence-based first-line monotherapy can be proposed. In this case the choice of the drug may depend on a variety of factors, including the approval status, labelling, route of administration, side-effect profile, potential interaction with background therapies, patient preferences, co-morbidities, physician experience and cost.

- Since head-to-head comparison between initial combination therapy with ambrisentan plus tadalafil has proven to be superior to initial monotherapy with ambrisentan or tadalafil in delaying clinical failure, a higher grade of recommendation has been given to this initial combination (Table 20) [247].

- In non-vasoreactive and treatment-naive patients at high risk (Table 13), initial combination therapy including i.v. prostacyclin analogues should be considered (Table 20). I.V. epoprostenol should be prioritised since it has reduced the 3-month rate of mortality in high-risk PAH patients also as monotherapy (Table 19) [149]. Alternative types of initial combination therapy may be considered (Table 20).

- In case of inadequate clinical response to initial combination therapy or initial monotherapy (Table 15), sequential double or triple combination therapy is recommended according to Table 21. The combination of riociguat and PDE-5i is contraindicated.

- In case of inadequate clinical response with sequential double combination therapy, triple combination therapy should be attempted (Tables 20 and 21).

- It seems reasonable to consider eligibility for lung transplantation after an inadequate clinical response to the initial monotherapy or initial combination therapy and to refer the patient for lung transplantation soon after the inadequate clinical response is confirmed on maximal combination therapy. BAS should be regarded as a palliative or bridging procedure in patients deteriorating despite maximal medical therapy.

\subsubsection{Diagnosis and treatment of pulmonary arterial hypertension complications 6.3.10.1 Arrhythmias}

Arrhythmias are an increasing clinical problem in PAH patients. In particular, the presence of symptomatic atrial arrhythmia might portend a poor prognosis [275]. In contrast to patients with LHD, malignant ventricular arrhythmias such as ventricular tachycardia, ventricular flutter and ventricular fibrillation are rare in PAH patients. In a series of 132 witnessed cardiac arrests in patients with PAH, ventricular fibrillation was observed in only $8 \%$ of the cases [276]. Another series of 231 patients with PAH or CTEPH followed over a 6-year period did not report any case of malignant ventricular arrhythmia [275]. In that series, supraventricular tachyarrhythmias occurred with an annual incidence of $2.8 \%$. Atrial flutter and atrial fibrillation were equally common and both arrhythmias invariably led to clinical deterioration with signs of right heart failure. Treatment of atrial flutter proved to be more successful than treatment of atrial fibrillation. Restoration of a stable sinus rhythm was associated with a favourable long-term survival, while persistent atrial fibrillation was associated with a 2-year mortality $>80 \%$ [275]. Supraventricular arrhythmias represent an indication for oral anticoagulation with vitamin $\mathrm{K}$ antagonists or new oral anticoagulants. Both electric cardioversion and radiofrequency ablation in refractory cases have proven to be effective [277]. 
Although prospective and controlled data are lacking, these findings suggest that maintenance of a stable sinus rhythm after cardioversion should be considered an important treatment goal in patients with PAH. In order to achieve a stable sinus rhythm, prophylaxis with antiarrhythmic drugs without negative inotropic effects, such as oral amiodarone (see interactions in Web Table VII), should also be considered, even if specific data regarding efficacy are lacking.

\subsubsection{Haemoptysis}

Haemoptysis is a recognised complication of $\mathrm{PH}$, represents a risk factor for mortality and appears to be more frequent in specific types, such as HPAH, PAH associated with CHD and CTEPH. Haemoptysis severity ranges from mild to very severe leading to sudden death. Reported prevalence is variable, from $1 \%$ to $6 \%$ [278]. Bronchial artery embolization is suggested as an acute emergency procedure in the case of severe haemoptysis or as elective intervention in cases of frequent mild or moderate episodes. Haemoptysis may represent a contraindication to anticoagulant therapy.

\subsubsection{Mechanical complications}

Mechanical complications in PAH patients are usually related to progressive dilatation of the PA and include PA aneurysms, rupture and dissection and compression of different intrathoracic structures such as the left main coronary artery, pulmonary veins, main bronchi and the recurrent laryngeal nerves [279-281]. Symptoms and signs are not specific and are variable according to the different complications, including chest pain (angina-like or not), dyspnoea, regional lung oedema and sudden death. Contrast-enhanced and high-resolution CT scan represent the best imaging option to detect mechanical complications.

Treatment options are not well established in cases of PA aneurysms, pseudo-aneurysms and dissection $[279,280]$.

The indications and results of a traditional surgical approach in the presence of $\mathrm{PH}$ are unknown and the predictable risks may be very high. The percutaneous approach (stenting) faces relevant technical issues mainly due to early branching of the main and lobar PA. A possible approach is double-lung or heartlung transplantation, but this solution can be applied only in chronic stabilised cases, and clear indications are lacking. In patients with left main coronary artery compression, a percutaneous stenting procedure should be performed [282].

\subsubsection{End of life care and ethical issues}

The clinical course of $\mathrm{PH}$ is one of progressive deterioration interspersed with episodes of acute decompensation. It is difficult to predict when patients will die since death may come either suddenly or slowly because of progressive heart failure. It has been shown that physicians caring for patients tend to be overoptimistic in their prognostication and frequently misunderstand their patients' wishes.

Open and sensitive communication with patients allows advanced planning and discussion of their fears, concerns and wishes, which is essential to good care. Opportunities to discuss prognosis should be created at the time of initial diagnosis. Recognition that cardiopulmonary resuscitation in severe $\mathrm{PH}$ has a poor outcome may enable a do-not-resuscitate order. This may increase the chance of patients being in their preferred place of care at the end of life.

Patients approaching the end of life require frequent assessment of their full needs by a multidisciplinary team. Attention should be given to controlling the distressing symptoms and prescribing appropriate drugs while withdrawing unnecessary medication. Well-informed psychological, social and spiritual support is also vital. Specialist palliative care should be consulted about patients whose needs are beyond the expertise of the $\mathrm{PH}$ team.

$\mathrm{PH}$ is a disease that may be severely life-limiting. In addition to psychological and social support, there should be proactive advanced care planning with referral to specialist palliative care services when appropriate.

\section{Specific pulmonary (arterial) hypertension subsets \\ 7.1 Paediatric pulmonary arterial hypertension}

$\mathrm{PH}$ can present at any age from the neonatal period to adulthood. Paediatric $\mathrm{PH}$ has some unique features that are not found in adult $\mathrm{PH}$, including prenatal aetiological factors, and postnatal parenchymal and vascular abnormalities in lung development [283]. Before the epoprostenol era, the prognosis had been worse in children, with a median survival estimated at 10 months, compared to 2.8 years in adults; however, with new targeted therapies the outcome has improved significantly $[9,284]$.

Recent data from registries have shed light on the prevalence and incidence of paediatric $\mathrm{PH}$. In the Netherlands, the incidence and prevalence of IPAH is 0.7 and 4.4 per million children, respectively [285]. Similar numbers have been observed in the UK, with annual cases of IPAH of 0.48 per million and a 
prevalence of 2.1 per million [286]. National and large-scale registries either including children [284] or exclusively dedicated to paediatrics $[287,288]$ have described the different aetiologies of PH, with IPAH, $\mathrm{HPAH}$ and $\mathrm{CHD}-\mathrm{PAH}$ as the most common. However, $\mathrm{PH}$ associated with respiratory disease is also noted to be important and may be underreported [287].

Although the 2009 Dana Point classification included most of the paediatric causes, it was felt that paediatric aetiologies should be better described. The 2011 Panama classification was recently proposed, with 10 different subgroups of paediatric PH [289]. The 2013 Nice classification includes new groups and aetiologies specific to children [9], such as congenital and acquired left heart inflow/outflow tract obstruction and segmental PH; it has been further adapted for the current ESC/ERS Guidelines (Table 4 and Web Table I) with provision of clinical (Table 6) and anatomical-pathophysiological classification of congenital systemic-to-pulmonary shunts associated with PAH (Web Table II) and of developmental lung disease (Web Table III)

PPHN remains in the PAH group but has been moved to a subgroup, as it is considered to be a specific entity with a more transient course (Table 4 and Web Table IV) in most cases.

\subsubsection{Diagnosis}

Dyspnoea, fatigue and failure to thrive are common symptoms; syncope is more common in children, but overt RV failure is a late event and the child may die of sudden death before the occurrence of RV failure $[284,287]$. A specific diagnostic workup has been recently recommended, and even if some causes are rare, they should be excluded before a definite diagnosis of IPAH [284]. A thorough family and personal history, including pregnancy, delivery and postnatal details, is essential [290]. Diagnosis must be confirmed by heart catheterization, and vasoreactivity testing should be performed. Recent reports suggest that cardiac catheterization may be a greater risk in children compared with adults, with younger age $(<1$ year) and worse FC (WHO-FC IV) as risk factors. Cardiac catheterization performed in expert centres is recommended [290]. The general scheme of the diagnostic algorithm for adult patients with $\mathrm{PH}$ (Figure 1) may also be adopted in children, with some adaptation related to the different epidemiology [9].

As in adults, clinical evidence of RV failure, progression of symptoms, WHO-FC III/IV and elevated BNP levels are recognized as being associated with higher risk of death. In children, failure to thrive, haemodynamic parameters such as the PAPm:systemic artery pressure ratio, RAP $>10 \mathrm{mmHg}$ and PVR index $>20 \mathrm{WU} / \mathrm{m}^{2}$ have also been associated with a higher risk of death, while the $6 \mathrm{MWD}$ was not a prognostic parameter.

\subsubsection{Therapy}

There is a lack of randomized trials in paediatrics, making it difficult to deliver strong guidelines [291, 292]. A specific treatment algorithm, similar to that used in adults (Figure 2), has been recommended. Determinants of risk and risk stratification have also been suggested [9]. CCBs are used in responders, but close follow-up is mandatory, as some patients may fail long-term therapy.

Indications for epoprostenol are similar to those for adults. The optimal dose varies between patients and thus individual adaptation is required [293, 294]. Use of i.v. iloprost and trepostinil, as well as subcutaneous trepostinil [295], has been reported. Oral beraprost is used in some countries, but lack of proof of efficacy is a problem. Inhaled iloprost is difficult to deliver, but some reports have shown efficacy, mostly in combination with other therapies [296].

Bosentan pharmacokinetics have been assessed in two studies [297, 298]. Several uncontrolled studies have shown positive results similar to adults, with survival rates around $80-90 \%$ at 1 year [298]. A paediatric formulation is available in Europe [299]. Data with ambrisentan are scarce and a study is ongoing.

Sildenafil has shown efficacy [300] and has been approved in Europe for children 1-17 years of age. Increased mortality using high doses has raised concerns; therefore high doses should not be used in children (high individual doses of sildenafil on a three daily dosing not recommended: $>10 \mathrm{mg} / \mathrm{dose}$ with a bodyweight of $8-20 \mathrm{~kg},>20 \mathrm{mg} /$ dose in children with a bodyweight $>20 \mathrm{~kg}$ or $>1 \mathrm{mg} / \mathrm{kg} / \mathrm{dose}$ in infants and small children) [301].

Data on tadalafil also suggest efficacy [302]; a trial is currently under way to define the specific dosage for children.

An increasing number of paediatric patients are on combination therapies even though evidence is still limited [303].

RV decompression strategies include atrioseptostomy [304], ductal stenting in cases of patent ductus arteriosus [305] and a surgical Potts shunt [306]. Transcatheter Potts shunt creation has also been proposed [307]. Lung transplantation remains an important option for paediatric $\mathrm{PH}$ patients. 
Specific goals for treatment should be applied for children. Some have been extrapolated from defined risk factors in children but still require validation in large cohorts [9]. Recently FC, TAPSE and NT-proBNP have been recognized as treatment goals [308].

The recommendations for paediatric $\mathrm{PH}$ are reported in the Table 23.

\subsection{Pulmonary arterial hypertension associated with adult congenital heart disease}

$\mathrm{PAH}$ associated with adult CHD is included in group 1 of the $\mathrm{PH}$ clinical classification (Table 4) and represents a very heterogeneous patient population. A specific clinical classification (Table 6) and an anatomical-pathophysiological classification (Web Table II) are provided to better characterize each individual patient with PAH associated with adult CHD $[13,309]$. Some malformations, such as patent ductus arteriosus, sinus venosus atrial septal defect or partial anomalous pulmonary venous return, are often concealed and patients are misclassified as suffering from IPAH. Hence these congenital anomalies should be specifically sought.

Epidemiological data remain scarce, as no studies have been designed to assess the prevalence of PAH in adult $\mathrm{CHD}$, although the figure of $5-10 \%$ was reported in a European survey [310]. Persistent exposure of the pulmonary vasculature to increased blood flow due to systemic-to-pulmonary shunts, as well as increased pressure, may result in a typical pulmonary obstructive arteriopathy (identical to other PAH forms) leading to an increase in PVR. If PVR approaches or exceeds systemic vascular resistance (SVR), the shunt is reversed (Eisenmenger syndrome) [311].

\subsubsection{Diagnosis}

As indicated in Table 6, the clinical presentation of adult patients with PAH may differ. Eisenmenger syndrome is a multisystem disorder and the most severe form of PAH in adult CHD. The signs and symptoms of Eisenmenger syndrome result from $\mathrm{PH}$, low arterial $\mathrm{O}_{2}$ saturation and haematological changes, including secondary erythrocytosis, thrombocytopenia and at times leucopenia. They include dyspnoea, fatigue and syncope. In patients with $\mathrm{PAH}$ associated with adult $\mathrm{CHD}$ without reversal of the shunt, the degree of cyanosis and erythrocytosis may be mild to moderate. Eisenmenger syndrome patients also present with haemoptysis, cerebrovascular accidents, brain abscesses, coagulation abnormalities and sudden death. Subjects with Eisenmenger syndrome have reduced life expectancy, although many survive into their third or fourth decade, with some even surviving up to their seventh decade [312]. In patients listed for lung or heart-lung transplantation when no medical treatment was available, Eisenmenger syndrome had better survival compared with IPAH, with a 3-year survival rate of $77 \%$ compared with $35 \%$ for untreated IPAH [313]. In a recent study on the different clinical groups of PAH patients associated with CHD (Table 6), the worst survival was observed in patients with PAH after defect repair or with small/coincidental defects as compared with patients with Eisenmenger syndrome or those with prevalent systemic-to-pulmonary shunts [314]. Improved survival may result from preservation of RV function, as the RV does not undergo remodelling at birth and remains hypertrophied [315]. The RV is also relieved by the right-to-left shunt, sustaining $\mathrm{CO}$ at the expense of hypoxaemia and cyanosis.

Of all patients with $\mathrm{CHD}$, those with Eisenmenger syndrome are the most severely compromised in terms of exercise intolerance $[314,316]$.

Patients with CHD (in particular those without shunts) may also develop PH due to LHD (group 2, Table 4) or concomitant lung diseases (group 3, Table 4). In these cases, a comprehensive diagnostic workup, as reported in the section 7.1.1, is recommended.

\subsubsection{Therapy}

Operability may be considered in patients with prevalent systemic-to-pulmonary shunting (Table 6). The criteria for shunt closure based on baseline PVR have been proposed (Table 24) based on available literature data [317-319]. Additional criteria include the type of the defect, age, the PVR:SVR ratio and the Qp:Qs ratio [320]. No prospective data are available on the usefulness of vasoreactivity testing, closure test or lung biopsy for operability assessment [320]. Surgical or percutaneous intervention is contraindicated in patients with Eisenmenger syndrome and likely is useless in patients with small/coincidental defects.

The medical treatment strategy for patients with $\mathrm{PAH}$ associated with $\mathrm{CHD}$, and in particular for subjects with Eisenmenger syndrome, is mainly based on the clinical experience of experts rather than being formally evidence-based [311]. A specific treatment algorithm has been proposed [309].

PAH associated with CHD patients should be managed in specialised centres. Patient education, behavioural modifications and awareness of potential medical risk factors are important aspects of management. 
TABLE 23 Recommendations for paediatric pulmonary hypertension

\begin{tabular}{|l|c|c|c|}
\hline Recommendations & Class $^{\mathrm{a}}$ & Level $^{\mathrm{b}}$ & Ref.c $^{\mathrm{c}}$ \\
\hline $\begin{array}{l}\text { A PH diagnostic algorithm workup is } \\
\text { recommended for diagnosis and } \\
\text { definition of the specific aetiology } \\
\text { group in paediatric PH patients }\end{array}$ & $\mathrm{I}$ & $\mathrm{C}$ & {$[9,290]$} \\
\hline $\begin{array}{l}\text { A PAH-specific therapeutic algorithm is } \\
\text { recommended in paediatric PH } \\
\text { patients }\end{array}$ & $\mathrm{I}$ & $\mathrm{C}$ & {$[9]$} \\
\hline $\begin{array}{c}\text { Combination therapy should be } \\
\text { considered in paediatric PH patients }\end{array}$ & Ila & C & $\begin{array}{c}{[295,298,} \\
302]\end{array}$ \\
\hline $\begin{array}{c}\text { Specific paediatric determinants of risk } \\
\text { should be consideredd }\end{array}$ & Ila & C & {$[9,308]$} \\
\hline
\end{tabular}

PAH: pulmonary arterial hypertension; $\mathrm{PH}$ : pulmonary hypertension. ${ }^{a}$ Class of recommendation. ${ }^{b}$ Level of evidence.

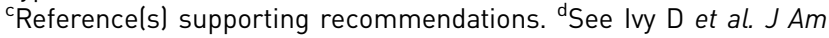
Coll Cardiol 2013; 62(25): D117-D126.
TABLE 24 Recommendations for correction of congenital heart disease with prevalent systemic-to-pulmonary shunts

\begin{tabular}{|c|c|c|c|c|c|}
\hline \multicolumn{3}{|c|}{ Recommendations } & \multirow{2}{*}{ Class $^{a}$} & \multirow{2}{*}{ Levelb } & \multirow[b]{2}{*}{ Ref. $^{c}$} \\
\hline $\begin{array}{c}\text { PVRi } \\
\left(\mathrm{WU} \cdot \mathrm{m}^{2}\right)\end{array}$ & $\begin{array}{l}\text { PVR } \\
\text { (WU) }\end{array}$ & Correctabled & & & \\
\hline$<4$ & $<2.3$ & Yes & Ila & C & [317] \\
\hline$>8$ & $>4.6$ & No & Ila & C & [317] \\
\hline $4-8$ & $2.3-4.6$ & $\begin{array}{c}\text { Individual } \\
\text { patient } \\
\text { evaluation in } \\
\text { tertiary } \\
\text { centres }\end{array}$ & Ila & C & [317] \\
\hline
\end{tabular}

PVR: pulmonary vascular resistance; PVRi: pulmonary vascular resistance index; WU: Wood units. ${ }^{a}$ Class of recommendation. ${ }^{b}$ Level of evidence. ${ }^{\mathrm{c}}$ Referencels) supporting recommendations. ${ }^{d}$ With surgery or intravascular percutaneous procedure.

Patients with PAH associated with CHD may present with clinical deterioration in different circumstances, such as during non-cardiac surgery requiring general anaesthesia, dehydration, lung infections and at high altitudes. It is recommended to avoid strenuous exercise, but mild activities seem to be beneficial. Pregnancy is associated with very high risk to both the mother and foetus and should be discouraged. Hence effective contraception is mandatory. Dual contraception is indicated in patients taking ERAs in light of the interaction with progesterone-based compounds.

Long-term home $\mathrm{O}_{2}$ therapy may improve symptoms but has not been shown to modify survival, at least when given only at night [179]. The use of supplemental $\mathrm{O}_{2}$ therapy is recommended in cases in which it produces a consistent increase in arterial $\mathrm{O}_{2}$ saturation and reduces symptoms.

The use of oral anticoagulant treatment in Eisenmenger syndrome is controversial: a high incidence of PA thrombosis and stroke is reported, but there is also an increased risk of haemorrhage and haemoptysis [321]. No data exist on this issue and thus definite recommendations cannot be made. Oral anticoagulant treatment may be considered in patients with PA thrombosis, signs of heart failure and absent or only mild haemoptysis [321].

Secondary erythrocytosis is beneficial for adequate $\mathrm{O}_{2}$ transport and delivery and routine phlebotomy should be avoided. If symptoms of hyperviscosity are present, phlebotomy with isovolumic replacement should be performed, usually when the haematocrit is $>65 \%$. Iron deficiency should be corrected. No clear data support the use of CCBs in patients with Eisenmenger syndrome and the empirical use of CCBs is dangerous and should be avoided.

One RCT is available with specific drug therapy in Eisenmenger syndrome patients: bosentan has been shown to improve 6MWT and decrease PVR after 16 weeks of treatment in WHO-FC III patients. Although a beneficial effect of bosentan has been shown on exercise capacity and quality of life in this group of patients, an effect on mortality remains uncertain [200]. Long-term follow-up (40 weeks) showed sustained improvement [322]. Bosentan is currently approved in Europe for WHO-FC III Eisenmenger syndrome patients.

Experiences with other ERAs [323] and the PDE-5is sildenafil [314] and tadalafil [324] show favourable functional and haemodynamic results in patients with $\mathrm{PAH}$ associated with $\mathrm{CHD}$ and Eisenmenger syndrome.

The use of i.v. epoprostenol has been reported in Eisenmenger syndrome patients, with favourable effects on haemodynamics and exercise capacity, although central lines expose the patients to the risk of paradoxical embolism and sepsis [223]. No data are available on the use of other prostanoids.

There are a few published data on combination therapy, but the rationale is the same as in IPAH [207, 314]. The use of PAH therapy to achieve the operability criteria in PAH with systemic-to-pulmonary cardiovascular shunts (Table 24), allowing defect correction ('treat-to-close' concept), is not supported by available data.

Heart-lung or lung transplantation with heart surgery is an option in special cases not responsive to medical treatment, but is limited by organ availability. Short- and long-term survival rates following heart- 
lung transplantation are similar to other forms of PAH. The prolonged estimated survival of patients with Eisenmenger syndrome makes the decision as to if and when patients should be listed difficult [309].

Recommendations for PAH associated with CHD are reported in the Table 25.

\subsection{Pulmonary arterial hypertension associated with connective tissue disease}

$\mathrm{PAH}$ is a well-known complication of CTDs such as SSc, systemic lupus erythematosus, mixed CTD and, to a lesser extent, rheumatoid arthritis, dermatomyositis and Sjögren's syndrome [325-329]. PAH associated with CTD is the second most prevalent type of PAH after IPAH in Western countries [10]. SSc, particularly in its limited variant, represents the main CTD associated with PAH in Europe and the USA (systemic lupus erythematosus is more common in Asia) $[325,329]$. The prevalence of haemodynamically proven pre-capillary $\mathrm{PH}$ in large cohorts of SSc patients ranges from 5 to $12 \%$ [46, 325, 330, 331]. In these patients, PH may occur in association with interstitial lung disease or as a result of an isolated pulmonary vascular disease, which may affect pre-capillary arterioles (PAH) and post-capillary venules (PVOD) [326, 332]. In addition, group 2 pulmonary venous hypertension from LHD may be present $[76,326,333]$. It is thus imperative to determine which mechanism is operative since this dictates treatment in the context of a multifaceted disease.

TABLE 25 Recommendations for pulmonary arterial hypertension associated with congenital heart disease

\begin{tabular}{|c|c|c|c|}
\hline Recommendations & Class $^{a}$ & Levelb $^{b}$ & Ref.c \\
\hline $\begin{array}{l}\text { Bosentan is recommended in } \\
\text { WHO-FC III patients with Eisenmenger } \\
\text { syndrome }\end{array}$ & 1 & B & {$[200,322]$} \\
\hline $\begin{array}{l}\text { Other ERAs, PDE-5is and prostanoids } \\
\text { should be considered in patients with } \\
\text { Eisenmenger syndrome }\end{array}$ & Ila & C & $\begin{array}{l}{[223,314} \\
323,324]\end{array}$ \\
\hline $\begin{array}{l}\text { In the absence of significant } \\
\text { haemoptysis, oral anticoagulant } \\
\text { treatment may be considered in } \\
\text { patients with PA thrombosis or signs } \\
\text { of heart failure }\end{array}$ & IIb & C & \\
\hline $\begin{array}{l}\text { The use of supplemental } \mathrm{O}_{2} \text { therapy } \\
\text { should be considered in cases in which } \\
\text { it produces a consistent increase in } \\
\text { arterial } \mathrm{O}_{2} \text { saturation and reduces } \\
\text { symptoms }\end{array}$ & Ila & C & [179] \\
\hline $\begin{array}{l}\text { If symptoms of hyperviscosity are } \\
\text { present, phlebotomy with isovolumic } \\
\text { replacement should be considered, } \\
\text { usually when the haematocrit is }>65 \%\end{array}$ & Ila & C & [183] \\
\hline $\begin{array}{l}\text { The use of supplemental iron treatment } \\
\text { may be considered in patients with low } \\
\text { ferritin plasma levels }\end{array}$ & $\mathrm{IIb}$ & C & [184] \\
\hline $\begin{array}{l}\text { Combination drug therapy may be } \\
\text { considered in patients with } \\
\text { Eisenmenger syndrome }\end{array}$ & IIb & C & {$[207,314]$} \\
\hline $\begin{array}{l}\text { The use of CCBs is not recommended } \\
\text { in patients with Eisenmenger } \\
\text { syndrome }\end{array}$ & III & C & [189] \\
\hline
\end{tabular}

CCBs: calcium channel blockers; ERAs: endothelin receptor antagonists; $\mathrm{O}_{2}$ : oxygen; PA: pulmonary artery; PDE-5is: phosphodiesterase type 5 inhibitors; WHO-FC: World Health Organization functional class. ${ }^{a}$ Class of recommendation. ${ }^{b}$ Level of evidence. ${ }^{\mathrm{C}}$ Reference(s) supporting recommendations. 


\subsubsection{Diagnosis}

Compared with IPAH, patients with CTD and PAH are mainly women (female:male ratio 4:1), are older (mean age at diagnosis $>60$ years), may present concomitant disorders (interstitial lung disease, LHD) and have shorter survival times [326, 330, 334-336]. The unadjusted risk of death for SSc-PAH compared with IPAH is 2.9 and the predictors of outcome are broadly similar as for IPAH [336]. Symptoms and clinical presentation are very similar to IPAH and occasional patients thought to have IPAH can be identified as having an associated CTD by immunological screening tests. High-resolution tomography of the chest is helpful for evaluating the presence of associated interstitial lung disease and/or PVOD [326, 332, 337]. An isolated reduction of DLCO is a frequent abnormality in SSc associated with PAH $[327,338]$.

Resting echocardiography is recommended as a screening test in asymptomatic SSc patients, followed by annual screening with echocardiography, DLCO and biomarkers [325]. A two-step composite score has been proposed in the DETECT study to select patients who should have RHC [327]. Specific recommendations for screening/early detection are provided in Web Table IX. The cost-effectiveness of these strategies has not been clarified as compared with symptom-based detection. Echocardiography is recommended in the presence of symptoms in other CTDs. As in other forms of PAH, RHC is recommended in all cases of suspected PAH associated with CTD to confirm the diagnosis, determine severity and rule out left-sided heart disease.

\subsubsection{Therapy}

Treatment of patients with CTD-associated PAH is more complex than that of IPAH. Immunosuppressive therapy combining glucocorticosteroids and cyclophosphamide may result in clinical improvement in patients with PAH associated with systemic lupus erythematosus or mixed CTD [339].

Long-term favourable response to CCB treatment is reported in $<1 \%$ of cases [189]. In SSc, the long-term risk: benefit ratio of oral anticoagulation is less favourable than in IPAH because of an increased risk of bleeding [175].

Treatment of patients with CTD and PAH should follow the same treatment algorithm as in IPAH (Figure 2). This recommendation is based on the fact that patients with CTD have been included in most of the major RCTs for regulatory approval of PAH therapy, including those with combination therapy.

Subgroup analysis of patients with SSc enrolled in the RCTs performed with bosentan, macitentan, sildenafil, riociguat and subcutaneous treprostinil have shown favourable effects. In some of these trials the magnitude of the response in the PAH subgroup associated with CTD was lower than in IPAH [340]. The choice of PAH therapy in the context of SSc and its generalized microangiopathy must take into account other vascular damage such as past/current digital ulcers.

Continuous i.v. epoprostenol therapy was shown to improve exercise capacity, symptoms and haemodynamics in a 3-month RCT in SSc-PAH [222]. However, retrospective analysis shows a better effect of i.v. epoprostenol on survival in IPAH as compared with SSc-PAH. This may be due, at least in part, to co-morbidities such as cardiopulmonary involvement.

SSc should not be considered as an a priori contraindication for lung transplantation [341]. A multidisciplinary approach optimizing SSc patient management before, during and after surgery is recommended [341]. Indications and contraindications for transplantation have to be adapted to the specificities of SSc with a special focus on digestive (gastro-oesophageal reflux disease and intestinal disease), cardiac, renal and cutaneous involvement.

The recommendations for PAH associated with CTD are reported in the Table 26.

\subsection{Pulmonary arterial hypertension associated with portal hypertension}

$\mathrm{PAH}$ associated with portal hypertension is commonly referred to as PoPH. This entity should not be confused with hepatopulmonary syndrome, which is characterized by abnormal pulmonary vasodilation and hypoxaemia [342]. However, overlaps between both conditions may occur [343]. As implied by the term, PoPH is associated with the presence of portal hypertension, not necessarily with the presence of liver disease. However, as cirrhotic liver disease is by far the most common cause of portal hypertension, $\mathrm{PoPH}$ is most frequently encountered in patients with cirrhosis. Approximately $1-5 \%$ of patients with portal hypertension develop PAH [344] and the risk seems to be largely independent from the aetiology of liver disease and from the impairment of hepatic function [345]. Although some genetic risk factors have been described [346], the pathogenetic link between portal and $\mathrm{PH}$ remains unclear.

\subsubsection{Diagnosis}

In general, the signs and symptoms of PoPH are similar or identical to most other forms of PAH, with progressive dyspnoea on exertion being the chief complaint. The clinical picture may be obscured by the 
TABLE 26 Recommendations for pulmonary arterial hypertension associated with connective tissue disease

\begin{tabular}{|l|c|c|c|}
\hline Recommendations & Class $^{\mathrm{a}}$ & Level $^{\mathrm{b}}$ & Ref.c $^{\mathrm{c}}$ \\
\hline $\begin{array}{l}\text { In patients with PAH associated with } \\
\text { CTD, the same treatment algorithm } \\
\text { as for patients with IPAH is } \\
\text { recommended }\end{array}$ & I & C & {$[46]$} \\
\hline $\begin{array}{l}\text { Resting echocardiography is } \\
\text { recommended as a screening test in } \\
\text { asymptomatic patients with SSc, } \\
\text { followed by annual screening with } \\
\text { echocardiography, DLCO and } \\
\text { biomarkers }\end{array}$ & I & C & {$[46]$} \\
\hline $\begin{array}{l}\text { RHC is recommended in all cases of } \\
\text { suspected PAH associated with CTD }\end{array}$ & I & C & {$[46,327]$} \\
\hline $\begin{array}{l}\text { Oral anticoagulation may be } \\
\text { considered on an individual basis } \\
\text { and in the presence of } \\
\text { thrombophilic predisposition }\end{array}$ & Ilb & C & {$[175,339]$} \\
\hline
\end{tabular}

CTD: connective tissue disease; DLCO: diffusing capacity of the lung for carbon monoxide; IPAH: idiopathic pulmonary arterial hypertension; PAH: pulmonary arterial hypertension; RHC: right heart catheterization; SSc: systemic sclerosis. ${ }^{a}$ Class of recommendation. ${ }^{\mathrm{b}}$ Level of evidence. ${ }^{\mathrm{c}}$ Referencels) supporting recommendations.

presence and severity of the underlying liver disease. The diagnostic assessment follows the same recommendations as for other forms of $\mathrm{PH}$, keeping in mind that the co-existence of portal hypertension and $\mathrm{PH}$ does not mean that these patients necessarily suffer from $\mathrm{PoPH}$ [344]. A complete diagnostic workup including RHC is required to assess disease severity, haemodynamic profile and other potential causes of $\mathrm{PH}$, including lung disease, LHD or chronic thromboembolic disease. As a group, patients with PoPH tend to have a higher CI and a lower PVR than patients with IPAH [347], but there is a wide overlap.

\subsubsection{Therapy}

The mortality risk of patients with PoPH is at least as high as in IPAH $[347,348]$ and these patients should be referred to centres with expertise in managing both $\mathrm{PAH}$ and liver disease. In general, treatment follows the general rules for other forms of $\mathrm{PAH}$, but there are important considerations. Patients with $\mathrm{PoPH}$ often have an elevated bleeding risk and anticoagulation is usually not recommended for these patients. Beta-blockers, which are frequently used to lower the portal pressure, should be avoided in patients with $\mathrm{PoPH}$, as they worsen haemodynamics and exercise in this patient population [349].

Patients with PoPH have been excluded from almost all RCTs in the PAH field (except for the PATENT study, which included 13 patients with PoPH). Anecdotal reports suggest that ERAs, PDE-5is, sGC stimulators and prostacyclin analogues may be used in this patient population [214, 350-356]. This includes potentially hepatotoxic drugs such as bosentan, but it should be noted that this compound tends to accumulate in patients with severely impaired liver function (i.e. Child-Pugh class B and C) [356]. Newer ERAs (ambrisentan, macitentan) have a theoretical advantage over bosentan, as the risk of drug-associated liver toxicity is lower [194, 201, 357], but both drugs have not been systematically evaluated in patients with PoPH.

The presence of $\mathrm{PH}$ is of particular importance in patients undergoing liver transplantation [358]. Mild $\mathrm{PH}$ with normal or near-normal PVR in the presence of high $\mathrm{CO}$ is usually well tolerated and tends to be reversible after transplantation [359]. $\mathrm{PAH}$, in contrast, has been identified as a major risk factor in the setting of liver transplantation. In a series from the Mayo Clinic, the mortality rate was $100 \%$ in patients with a PAPm $\geqslant 50 \mathrm{mmHg}$ and $50 \%$ in patients with a PAPm between 35 and $50 \mathrm{mmHg}$ and a PVR $\geqslant 250$ dyn $\cdot s \cdot \mathrm{cm}^{-5}$ [359]. Therefore patients evaluated for liver transplantation should be screened for signs of $\mathrm{PH}$, even in the absence of corresponding clinical symptoms. In the past, significant PAH was considered a contraindication for liver transplantation, but anecdotal reports suggest that pretreating these patients with 
PAH drugs might improve the outcome after liver transplantation [360-363]. There is not yet sufficient evidence to allow general recommendations and the transplant decision should be made by multidisciplinary teams in expert centres. A few specialized centres offer combined liver-lung or liverheart-lung transplantation for carefully selected patients [364].

The recommendations for PAH associated with portal hypertension are reported in the Table 27.

\subsection{Pulmonary arterial hypertension associated with human immunodeficiency virus infection}

The use of highly active antiretroviral therapy (HAART) and aggressive management of opportunistic infections has contributed to increased life expectancy in HIV-infected patients [366, 367]. Consequently, the spectrum of complications has shifted towards other long-term conditions, including PAH. It is likely that modern HIV management with HAART has also resulted in improved survival and decreased incidence of HIV-associated PAH [368]. Taken together, these effects on survival and incidence have resulted in a stable PAH prevalence in HIV-infected patients over recent decades. A population study indicated that the minimal prevalence of HIV-related PAH was $0.46 \%$, very similar to the prevalence before the HAART era [369]. The pathogenesis of HIV-related PAH remains unclear. The absence of viral particles in the complex plexiform lesions found from these patients suggests that an indirect action of viral infection on inflammation and growth factors may act as a trigger in a predisposed patient.

\subsubsection{Diagnosis}

HIV-associated PAH shares a similar clinical presentation with IPAH. At the time of diagnosis, a majority of patients are in an advanced New York Heart Association (NYHA)-FC III or IV. Patients may present

TABLE 27 Recommendations for pulmonary arterial hypertension associated with portal hypertension

\begin{tabular}{|l|c|c|c|}
\hline Recommendations & Class $^{\text {a }}$ & Level $^{\mathrm{b}}$ & Ref.c \\
\hline $\begin{array}{l}\text { Echocardiographic assessment for } \\
\text { signs of PH is recommended in } \\
\text { symptomatic patients with liver } \\
\text { disease or portal hypertension and } \\
\text { in all candidates for liver } \\
\text { transplantation }\end{array}$ & & & \\
\hline $\begin{array}{l}\text { It is recommended that patients } \\
\text { affected by PAH associated with } \\
\text { portal hypertension should be } \\
\text { referred to centres with expertise in } \\
\text { managing both conditions }\end{array}$ & I & B & [344] \\
\hline $\begin{array}{l}\text { It is recommended that the } \\
\text { treatment algorithm for patients } \\
\text { with other forms of PAH should be } \\
\text { applied to patients with PAH } \\
\text { associated with portal } \\
\text { hypertension, taking into account } \\
\text { the severity of liver disease }\end{array}$ & I & C & [214, \\
\hline $\begin{array}{l}\text { Anticoagulation is not recommended } \\
\text { in patients with PH associated with } \\
\text { portal hypertension }\end{array}$ & III & C & [365] \\
\hline $\begin{array}{l}\text { Liver transplantation may be } \\
\text { considered in selected patients } \\
\text { responding well to PAH therapy }\end{array}$ & IIb & C & [361-363] \\
\hline $\begin{array}{l}\text { Liver transplantation is } \\
\text { contraindicated in patients with } \\
\text { severe and uncontrolled PAH }\end{array}$ & III & C & {$[361-363]$} \\
\hline
\end{tabular}

$\mathrm{PAH}$ : pulmonary arterial hypertension; $\mathrm{PH}$ : pulmonary hypertension. ${ }^{a}$ Class of recommendation. ${ }^{b}$ Level of evidence. ${ }^{\mathrm{c}}$ Reference(s) supporting recommendations. 
with other risk factors for PAH, such as liver disease (chronic hepatitis B or C), exposure to drugs or toxins or PE due to i.v. drug abuse. Patients with HIV-associated PAH are more likely to be male and i.v. drug abusers. Animal research supports the notion that the additive effect of cocaine on HIV infection might play a role in the development of pulmonary arteriopathy. Because of its low prevalence, asymptomatic HIV-infected patients should not be screened for PAH. However, echocardiography must be performed in patients with unexplained dyspnoea to detect HIV-related cardiovascular complications such as myocarditis, cardiomyopathy or PAH. RHC confirmation is mandatory to establish the diagnosis of HIV-related PAH and the absence of LHD [369]. PAH is an independent risk factor for death in HIV-infected patients.

\subsubsection{Therapy}

In the absence of specific recommendations, treatment of HIV-PAH follows guidelines for the treatment of IPAH together with HAART. On multivariate analysis, a CI $>2.8 \mathrm{l} / \mathrm{min} / \mathrm{m}^{2}$ and a CD4 lymphocyte count $>200$ cells $/ \mathrm{ml}$ are independent predictors of survival [225]. Anticoagulation is not routinely recommended because of an increased risk of bleeding, anticipated compliance issues and drug interactions. Patients with HIV-related PAH appear to be non-responders to acute vasodilator challenge and thus should not receive CCBs [189]. Uncontrolled studies suggest that prostacyclins may improve exercise tolerance, haemodynamics and symptoms in HIV-related PAH [218]. An open-label study reported the effects of bosentan in patients with HIV-related PAH, showing an improvement in all efficacy measures, including 6MWT and invasive haemodynamics [370]. Sporadic cases have been included in the ambrisentan RCTs [194]. Hepatic tolerability was similar to previously reported observations in other forms of PAH. The interpretation of these studies is limited by the small sample size and their open-label nature. In the case of sildenafil, the dose should be reduced if ritonavir and saquinavir are co-administered due to drug-drug interactions. HIV infection is generally considered an exclusion criterion for lung transplantation, even though in some centres specific programmes have been implemented. Of note, cases of reversible disease have been described in HIV-PAH patients treated with HAART and specific therapies. This finding, together with the decreased incidence of HIV-PH in the modern management era, may indicate that aggressive management improves outcomes in this patient population. Further studies are needed to understand the underlying reasons of improved outcomes in these patients.

The recommendations for PAH associated with HIV infection are reported in the Table 28.

\subsection{Pulmonary veno-occlusive disease and pulmonary capillary haemangiomatosis}

Both PVOD and PCH are uncommon conditions but are increasingly recognized as causes of $\mathrm{PH}$ [92, 371]. Pathologic characteristics of $\mathrm{PCH}$ are found in 73\% of PVOD patients and, inversely, pathologic characteristics of PVOD are found in $80 \%$ of $\mathrm{PCH}$ patients [372]. Similarities in pathologic features and

TABLE 28 Recommendations for pulmonary arterial hypertension associated with human immunodeficiency virus infection

\begin{tabular}{|l|c|c|c|}
\hline Recommendations & Class $^{\mathrm{a}}$ & Level $^{\mathrm{b}}$ & Ref.c $^{\mathrm{c}}$ \\
\hline $\begin{array}{l}\text { Echocardiographic screening in } \\
\text { asymptomatic HIV patients to } \\
\text { detect PH is not recommended }\end{array}$ & III & C & [369] \\
\hline $\begin{array}{l}\text { In patients with PAH associated with } \\
\text { HIV infection, the same treatment } \\
\text { algorithm used for patients with }\end{array}$ & Ila & C & {$[194,367]$} \\
$\begin{array}{l}\text { PAH should be considered, taking } \\
\text { into consideration co-morbidities } \\
\text { and drug-drug interactions }\end{array}$ & & & \\
\hline $\begin{array}{l}\text { Anticoagulation is not } \\
\text { recommended because of a lack of } \\
\text { data on the efficacy:risk ratio }\end{array}$ & III & C & {$[175,367]$} \\
\hline
\end{tabular}

HIV: human immunodeficiency virus; IPAH: idiopathic pulmonary arterial hypertension; $\mathrm{PAH}$ : pulmonary arterial hypertension. ${ }^{a}$ Class of recommendation. ${ }^{b}$ Level of evidence. ${ }^{c}$ Reference(s) supporting recommendations. 
clinical characteristics and the risk of drug-induced pulmonary oedema with PAH therapy [371, 373] suggest that these two conditions overlap, and it has been proposed that $\mathrm{PCH}$ could be a secondary angioproliferative process caused by post-capillary obstruction of PVOD rather than a distinct disease [6, 372]. Thus PVOD and $\mathrm{PCH}$ have been classified together in a specific subgroup of the clinical classification next to PAH (Table 4, group 1') because of their pathological, genetic and clinical similarities and differences with PAH [6]. The true incidence of PVOD/PCH remains unknown because many cases are still misclassified as $\mathrm{PAH}$ [374]. The proportion of idiopathic cases of $\mathrm{PAH}$ that in reality fulfil the criteria for $\mathrm{PVOD} / \mathrm{PCH}$ is likely to be around $10 \%$ (lowest estimates of $\mathrm{PVOD} / \mathrm{PCH}$ incidence and prevalence are $<1$ case/million) $[92,374]$. In contrast to IPAH, there is a male predominance in PVOD and the prognosis appears to be worse [371]. Familial occurrence of PVOD has been reported, but BMPR2 mutations have rarely been found in patients with this disease $[28,371,375]$. While PAH that is due to $B M P R 2$ mutations segregates as an autosomal dominant trait with incomplete penetrance, familial cases of $\mathrm{PVOD} / \mathrm{PCH}$ typically occur in the young siblings of one generation with unaffected parents, indicating that the disease segregates as a recessive trait [28]. In these families, PVOD/PCH is caused by bi-allelic mutations in EIF2AK4 [28]. Like PAH, PVOD/PCH may complicate the course of associated conditions (SSc, HIV infection, etc.) and exposure to drugs or toxins (cyclophosphamide, mitomycin, etc.).

\subsubsection{Diagnosis}

The diagnosis of PVOD/PCH can be established with a high probability by the combination of clinical suspicion, physical examination, bronchoscopy and radiological findings [371]. This non-invasive approach may avoid the need to perform a hazardous lung biopsy (the gold standard to confirm a histological diagnosis of PVOD/PCH), which is no longer recommended in most cases [371]. In heritable cases, identification of a bi-allelic EIF2AK4 mutation is sufficient to confirm a diagnosis of PVOD/PCH without histological confirmation [28, 376]. Most patients complain of dyspnoea on exertion and fatigue, a clinical presentation that is indistinguishable from PAH [371, 374]. Physical examination may reveal digital clubbing and bi-basal crackles on lung auscultation, these being unusual in $\mathrm{PAH}$ [371]. Case series indicate that patients with PVOD/PCH are more severely hypoxaemic and have much lower DLCO levels than in other forms of PAH $[92,371]$. This can be explained by the presence of chronic interstitial pulmonary oedema and pulmonary capillary proliferation typical of PVOD/PCH.

Chest radiography may reveal Kerley B lines, mediastinal lymph node enlargement and peripheral interstitial infiltrate, in addition to other signs of $\mathrm{PH}[92,371]$. High-resolution CT of the chest is the investigation of choice. Typical findings suggestive of $\mathrm{PVOD} / \mathrm{PCH}$ are the presence of subpleural thickened septal lines, centrilobular ground-glass opacities and mediastinal lymphadenopathy [92, 371]. The association of these three findings was found to be $100 \%$ specific for $\mathrm{PVOD} / \mathrm{PCH}$ in cases of $\mathrm{PAH}$, with $66 \%$ sensitivity. In addition, their presence appears to closely correlate with the risk of pulmonary oedema with PAH drugs. V/Q lung scanning is not useful in discriminating PVOD/PCH from IPAH [377].

Because PVOD/PCH may be associated with occult alveolar haemorrhage, bronchoscopy with bronchoalveolar lavage may be a useful tool in the diagnostic strategy. In a retrospective study, the results of bronchoalveolar lavage performed in 19 patients with $\mathrm{PAH}$ or PVOD/PCH were analysed. Compared with IPAH, the eight cases of PVOD presented with a significantly elevated cell count, a higher percentage of hemosiderin-laden macrophages and a markedly elevated Golde score [378].

The haemodynamic presentation of PVOD is similar to IPAH [92, 371]. Importantly, PAWP is almost invariably normal, because the pathological changes occur in small venulae and capillaries and do not affect the larger pulmonary veins $[92,371]$. Vasoreactivity testing may be complicated by acute pulmonary oedema $[92,371]$.

\subsubsection{Therapy}

There is no established medical therapy for PVOD/PCH [92]. Most importantly, vasodilators and, in particular, i.v. epoprostenol must be used with great caution because of the high risk of severe drug-induced pulmonary oedema $[92,373]$. However, there are reports of sustained clinical improvement in individual patients treated with these medications [379]. High-dose diuretics, $\mathrm{O}_{2}$ therapy and slow increases in epoprostenol doses are recommended [379]. Therefore therapy for PVOD/PCH should be undertaken only at centres with extensive experience in the management of $\mathrm{PH}$ and patients should be fully informed about the risks. Investigational use of angiogenesis inhibitors such as interferon alfa-2a has been considered in these patients but is not currently recommended [379]. The only curative therapy for $\mathrm{PVOD} / \mathrm{PCH}$ is lung transplantation, and there are no reports of recurrence of disease following transplantation. Eligible patients with $\mathrm{PVOD} / \mathrm{PCH}$ should be referred to a transplant centre for evaluation as soon as the diagnosis is established [379].

The recommendations for PVOD/PCH are reported in the Table 29. 
TABLE 29 Recommendations for pulmonary veno-occlusive disease and pulmonary capillary haemangiomatosis

\begin{tabular}{|c|c|c|c|}
\hline Recommendations & Class $^{a}$ & Levelb & Ref.c \\
\hline $\begin{array}{l}\text { A combination of clinical findings, } \\
\text { physical examination, } \\
\text { bronchoscopy and radiological } \\
\text { findings is recommended to } \\
\text { diagnose PVOD/PCH }\end{array}$ & 1 & C & $\begin{array}{c}{[92,371} \\
378]\end{array}$ \\
\hline $\begin{array}{l}\text { Identification of a bi-allelic EIF2AK4 } \\
\text { mutation is recommended to } \\
\text { confirm a diagnosis of heritable } \\
\text { PVOD/PCH without histological } \\
\text { confirmation }\end{array}$ & I & B & {$[28,376]$} \\
\hline $\begin{array}{l}\text { Referral of eligible patients with } \\
\text { PVOD/PCH to a transplant centre } \\
\text { for evaluation is indicated as soon } \\
\text { as the diagnosis is established }\end{array}$ & 1 & C & [371] \\
\hline $\begin{array}{l}\text { Patients with PVOD/PCH should be } \\
\text { managed only in centres with } \\
\text { extensive experience in } \mathrm{PH} \text { due to } \\
\text { the risk of lung oedema after the } \\
\text { initiation of PAH therapy }\end{array}$ & Ila & C & $\begin{array}{c}{[371,373,} \\
379]\end{array}$ \\
\hline
\end{tabular}

PAH: pulmonary arterial hypertension; $\mathrm{PCH}$ : pulmonary capillary haemangiomatosis; $\mathrm{PH}$ : pulmonary hypertension; PVOD: pulmonary veno-occlusive disease. ${ }^{\mathrm{a}}$ Class of recommendation. ${ }^{b}$ Level of evidence. ${ }^{c}$ Referencels) supporting recommendations.

\section{Pulmonary hypertension due to left heart disease (group 2)}

$\mathrm{PH}$ is a common complication of LHDs (PH-LHD), frequently occurring as a 'symptom' of the underlying condition $[4,380]$. and often related to disease severity. PH-LHD can complicate any left heart disorder, such as valvular heart diseases and congenital defects. However, it has been most often studied in patients with heart failure and with preserved or reduced ejection fraction $[4,380]$. When present, PH-LHD results in more severe symptoms and worse exercise tolerance and exerts a negative impact on outcome [4, 380, 381]. Compared with PAH, patients with PH-LHD [especially in the case of heart failure with preserved LV ejection fraction (HF-pEF)] are often older, female, with a higher prevalence of cardiovascular co-morbidities [382] and most, if not all, of the features of metabolic syndrome [383].

The true prevalence of PH-LHD in heart failure remains unknown, mostly because the definition of $\mathrm{PH}$ in epidemiological studies has been based on echocardiography, with a variety of cut-off values [4, 384]. Invasive haemodynamics were reported in a limited number of single-centre reports [4]. In a retrospective analysis performed in a large $\mathrm{PH}$ centre, LHD was identified as the cause of $\mathrm{PH}$ in $36 \%$ of all patients referred for evaluation, of which $55 \%$ had 'passive' PH, defined as a TPG $<12 \mathrm{mmHg}[151,385]$.

PH develops in LHD in response to a passive backward transmission of filling pressures, mainly driven by LV diastolic function, enhanced by exercise-induced mitral regurgitation and a loss of LA compliance [4]. In some patients, these purely mechanical components of venous congestion may trigger a superimposed component, combining pulmonary vasoconstriction, decreased NO availability, increased endothelin expression, desensitization to natriuretic peptide-induced vasodilation and vascular remodelling [4, 380, 386]. This results in a further increase in PAPm in excess of the elevation of PAWP that may lead to pulmonary vascular disease, increased RV afterload and RV failure [4].

The definitions of $\mathrm{PH}$ and the difference between pre- and post-capillary $\mathrm{PH}$ are based on pressure cut-off. This explains why TPG, i.e. the difference between PAPm and PAWP, has been used to distinguish 'passive' PH (TPG $<12 \mathrm{mmHg}$ ) from 'reactive' PH (TPG $\geqslant 12 \mathrm{mmHg}$ ) [151]. However, this definition and the associated terminology have been unsatisfactory, to such an extent that 'out-of-proportion' PH-LHD has often been used to characterize a subset of patients with significant changes in pulmonary circulation $[4,151]$. Ideally the latter should be defined by a haemodynamic variable that would (i) be a marker of disease, (ii) be least dependent on changes in PAWP and stroke volume and (iii) take into account the 
pulsatile nature of the pulmonary circulation $[4,81]$. The TPG is influenced by all the determinants of PAPm, including flow, resistance and left heart filling pressure $[4,81,82]$. In contrast, diastolic PAP when compared with PAPs and PAPm is less influenced by PAWP at any level of stroke volume [4, 81]. Therefore the DPG (defined as diastolic PAP - mean PAWP) appears to best approach the characteristics required to determine pulmonary vascular disease $[4,81]$. In normal subjects, DPG lies in the $1-3 \mathrm{mmHg}$ range, and in patients evaluated for cardiac disease (excluding shunts), DPG remains $\leqslant 5 \mathrm{mmHg}$ in most cases $[4,81,387]$.

The role of DPG and TPG in predicting outcome in heart failure has recently been reported [83, 385, 388]. In a single-centre study of 3107 patients, a DPG $\geqslant 7 \mathrm{mmHg}$ has been reported to be associated with a worse prognosis in a subgroup of patients with increased TPG $>12 \mathrm{mmHg}$ [385]. In another study on 463 patients with LV ejection fraction $<40 \%$, the risk of death was higher in patients with $\mathrm{PH}$ when the definition was based on PVR $(P<0.01)$, with a higher mortality rate in patients with a PVR $\geqslant 3$ WU [387]. However, the outcome was not different whether the TPG was less than or greater than $12 \mathrm{mmHg}$ [83], suggesting that the latter could be less discriminant than PVR when $\mathrm{PH}$ is present. Interestingly, the authors reported that pulmonary arterial compliance was an independent marker of prognosis. Other investigators have also reported this observation, suggesting that PA compliance is altered in heart failure [389], even in the absence of PH [390]. In 25450 patients receiving a heart transplant in the USA, $22.6 \%$ were found to have a TPG $>12 \mathrm{mmHg}$ [83]. In this specific setting, the DPG was not found to predict a worse outcome at any level studied. Another recent study reported the lack of prognostic value of the DPG even if significant methodological limitations were raised [391]. Although limited by their retrospective nature, these studies do not provide a clear answer to the question of what is the best variable to predict outcome in PH-LHD. Therefore, for consistency with the general PH definition, it is recommended to use a combination of DPG and PVR to define the different types of PH-LHD, i.e. isolated post-capillary pulmonary hypertension (Ipc-PH) and combined post-capillary and pre-capillary pulmonary hypertension (Cpc-PH) (Table 3).

\subsection{Diagnosis}

In LHDs, and more specifically in left heart failure, $\mathrm{PH}$ can easily be suspected by a stepwise approach, combining clinical presentation, specific echocardiographic features and other modalities such as ECG and other imaging techniques. Although no single variable can differentiate PH-LHD from pre-capillary $\mathrm{PH}$, the presence of multiple risk factors and findings should raise suspicion for PH-LHD (Table 30). PH

TABLE 30 Examples of key factors suggestive of group 2 pulmonary hypertension

\begin{tabular}{|c|c|c|}
\hline Clinical presentation & Echocardiography & Other features \\
\hline Age $>65$ years & $\begin{array}{l}\text { Structural left heart abnormality } \\
\text { - Disease of left heart valves } \\
\text { - LA enlargement }(>4.2 \mathrm{~cm}) \\
\text { - Bowing of the IAS to the right } \\
\text { - LV dysfunction } \\
\text { - Concentric LV hypertrophy and/or increased LV mass }\end{array}$ & $\begin{array}{l}\text { ECG } \\
\text { - LVH and/or LAH } \\
\text { - AF/Afib } \\
\text { - LBBB } \\
\text { - Presence of Q waves }\end{array}$ \\
\hline $\begin{array}{l}\text { Symptoms of left heart } \\
\text { failure }\end{array}$ & $\begin{array}{l}\text { Doppler indices of increased filling pressures } \\
\text { - Increased E/e' } \\
\text { - >Type } 2-3 \text { mitral flow abnormality }\end{array}$ & $\begin{array}{l}\text { Other imaging } \\
\text { - Kerley B lines } \\
\text { - Pleural effusion } \\
\text { - Pulmonary oedema } \\
\text { - LA enlargement }\end{array}$ \\
\hline $\begin{array}{l}\text { Features of metabolic } \\
\text { syndrome }\end{array}$ & $\begin{array}{l}\text { Absence of } \\
\text { - RV dysfunction } \\
\text { - Mid systolic notching of the PA flow } \\
\text { - Percardial effusion }\end{array}$ & \\
\hline \multicolumn{3}{|l|}{$\begin{array}{l}\text { History of heart disease } \\
\text { (past or current) }\end{array}$} \\
\hline $\begin{array}{l}\text { Persistent atrial } \\
\text { fibrillation }\end{array}$ & & \\
\hline
\end{tabular}

AF: atrial flutter; Afib: atrial fibrillation; ECG: electrocardiogram; IAS: inter-atrial septum; LA: left atrium; LAH: left atrial hypertrophy/dilatation; LBBB: left bundle branch block; LV: left ventricle; LVH: left ventricular hypertrophy; PA: pulmonary artery; RV: right ventricle. 
should be suspected when patients present with otherwise unexplained symptoms, signs of right heart failure and co-morbidities associated with $\mathrm{PH}$, such as sleep apnoea syndrome, COPD, previous PE and risk factors for PAH.

The respective role of fluid loading and exercise testing to uncover PH-LHD in patients with HF-pEF is currently not standardized and normal values are lacking to provide a clear recommendation to clinicians. In addition, it has been suggested that patients with a diagnosis of PAH may present an abnormal increase in PAWP in response to fluid loading [77].

The indications for RHC in PH-LHD, preferably electively in stable condition, are reported in Table 10.

\subsection{Therapy}

The primary goal of therapy in PH-LHD must be to improve global management of the underlying condition prior to considering specific measures to treat $\mathrm{PH}$. This includes repair of valvular heart disease when indicated and aggressive therapy for heart failure with reduced systolic function [4, 392]. Some patients may also benefit from non-specific vasodilators such as nitrates and hydralazine, although evidence supporting this strategy is limited [4,392]. In severe heart failure, optimizing volume status is of critical importance and may require invasive monitoring [4, 393]. In addition, the implantation of an LV assist device has been shown to lower pulmonary pressures through LV unloading without increasing the risk of post-implantation RV failure $[4,394,395]$. Risk factors for cardiovascular diseases and features of metabolic syndrome should be controlled [4, 392]. Concomitant disorders leading to $\mathrm{PH}$ should be identified and treated, including COPD, sleep apnoea syndrome and PE. In contrast, there is no strong evidence-based recommendation for the treatment for HF-pEF [392].

The rationale to use PAH therapies in PH-LHD has been supported by acute or short-term studies using prostanoids, ERAs and PDE-5is. Most of these studies consistently reported improvements in haemodynamics, exercise capacity and symptoms [4, 396]. However, these studies carry significant methodological limitations (small sample size, single centre, unclear or no randomization process) that do not provide enough evidence to support the use of these drugs in the clinical management of patients [4]. In addition, there is no evidence suggesting that acute changes in pulmonary circulation may have a value outside of the setting of major cardiac surgery such as heart transplantation and/or LV assist device implantation $[4,380]$.

In a recent multicentre, placebo-controlled trial [397], 201 patients with $\mathrm{PH}$ due to systolic heart failure were randomized in four arms comparing three doses of riociguat $(0.5,1$ and $2 \mathrm{mg}$ t.i.d.) with placebo over 16 weeks. No effect on the primary endpoint (a change in PAPm after 16 weeks) was observed at any dose of riociguat compared with placebo [397].

Two multicentre clinical trials in PH-LHD are currently under way with sildenafil [SilHF (NCT01616381)] and macitentan [Melody-1 (NCT02070991)], the latter being the only study requiring RHC validation.

Therefore there is no new evidence supporting the use of PAH therapies in PH-LHD, due in part to the absence of studies specifically stratifying patients for $\mathrm{PH}$ and/or targeting this specific condition. Such trials are necessary to address this area of unmet medical need and patients with $\mathrm{Cpc}-\mathrm{PH}$ should be included in clinical trials. Recommendations for the management of PH-LHD are listed in Table 31.

\section{Pulmonary hypertension due to lung diseases and/or hypoxia (group 3)}

The pathology, pathophysiology and epidemiology of these conditions have been discussed previously (see section 4). The most common lung diseases associated with $\mathrm{PH}$ are COPD, interstitial lung disease and combined pulmonary fibrosis and emphysema (CPFE). Rare conditions such as Langerhans cell granulomatosis or sarcoidosis are reported in Web Table VIII. In any lung disease, the development of $\mathrm{PH}$ is accompanied by a deterioration of exercise capacity, worsening of hypoxaemia and shorter survival [398-400]. The severity of $\mathrm{PH}$ is usually poorly associated with the severity of the underlying lung disease $[401,402]$. The most common indicators for the presence of $\mathrm{PH}$ in these patients are a disproportionally low DLCO and a low $\mathrm{pCO}_{2}[401,402]$.

The haemodynamic classification of PH associated with lung disease is shown in Table 32 [20]. In case of severe $\mathrm{PH}$, other potential causes such as LHD or CTEPH should be excluded. In some patients with lung disease and $\mathrm{PH}$, especially in patients with mild lung disease but severe $\mathrm{PH}$, it may be difficult to determine whether $\mathrm{PH}$ is due to lung disease or whether the patient suffers from two diseases, i.e. PAH and chronic lung disease. Those patients should be referred to a $\mathrm{PH}$ centre where there is also expertise in lung diseases. 
TABLE 31 Management of pulmonary hypertension in left heart disease

\begin{tabular}{|l|c|c|c|}
\hline Recommendations & Class $^{\mathrm{a}}$ & Level $^{\mathrm{b}}$ & Ref.c $^{\mathrm{c}}$ \\
\hline $\begin{array}{l}\text { Optimization of the treatment of the } \\
\text { underlying condition is recommended } \\
\text { before considering assessment of PH-LHD } \\
\text { (i.e. treating structural heart disease) }\end{array}$ & I & B & [396] \\
\hline $\begin{array}{l}\text { It is recommended to identify other causes } \\
\text { of PH li.e. COPD, sleep apnoea syndrome, }\end{array}$ & & & \\
$\begin{array}{l}\text { PE, CTEPH) and to treat them when } \\
\text { appropriate before considering assessment } \\
\text { of PH-LHD }\end{array}$ & I & C & [344] \\
\hline $\begin{array}{l}\text { It is recommended to perform invasive } \\
\text { assessment of PH in patients on optimized } \\
\text { volume status }\end{array}$ & I & C & \\
\hline $\begin{array}{l}\text { Patients with PH-LHD and a severe } \\
\text { pre-capillary component as indicated by a } \\
\text { high DPG and/or high PVR should be } \\
\text { referred to an expert PH centre for a } \\
\text { complete diagnostic workup and an } \\
\text { individual treatment decision }\end{array}$ & Ila & C & \\
\hline $\begin{array}{l}\text { The importance and role of vasoreactivity } \\
\text { testing is not established in PH-LHD, } \\
\text { except in patients who are candidates for } \\
\text { heart transplantation and/or LV assist } \\
\text { device implantation }\end{array}$ & III & C & [396] \\
\hline $\begin{array}{l}\text { The use of PAH-approved therapies is not } \\
\text { recommended in PH-LHD }\end{array}$ & III & C & [396] \\
\hline
\end{tabular}

COPD: chronic obstructive pulmonary disease; CTEPH: chronic thromboembolic pulmonary hypertension; DPG: diastolic pressure gradient; LHD: left heart disease; LV: left ventricular; PE: pulmonary embolism; $\mathrm{PH}$ : pulmonary hypertension; PVR:

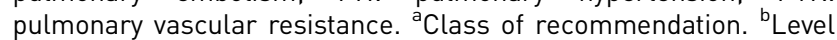
of evidence. ${ }^{C}$ Reference(s) supporting recommendations.
TABLE 32 Haemodynamic classification of pulmonary hypertension due to lung disease [9]

\begin{tabular}{|l|l|}
\hline Terminology & \multicolumn{1}{c|}{$\begin{array}{c}\text { Haemodynamics } \\
\text { (right heart catheterization) }\end{array}$} \\
\hline COPD/IPF/CPFE without PH & PAPm $<25 \mathrm{mmHg}$ \\
\hline COPD/IPF/CPFE with PH & PAPm $\geqslant 25 \mathrm{mmHg}$ \\
\hline COPD/IPF/CPFE with severe PH & $\begin{array}{l}\text { PAPm }>35 \mathrm{mmHg} \text {, or } \\
\mathrm{PAPm} \geqslant 25 \mathrm{mmHg} \text { in the presence } \\
\text { of a low cardiac output } \\
\text { (Cl }<2.5 \mathrm{~L} / \mathrm{min} / \mathrm{m}^{2} \text {, not explained } \\
\text { by other causes) }\end{array}$ \\
\hline
\end{tabular}

$\mathrm{Cl}$ : cardiac index; COPD: chronic obstructive pulmonary disease; CPFE: combined pulmonary fibrosis and emphysema; IPF: idiopathic pulmonary fibrosis; PAP: pulmonary artery pressure; PAPm: mean pulmonary arterial pressure; $\mathrm{PH}$ : pulmonary hypertension.

\subsection{Diagnosis}

Clinical symptoms and physical signs of $\mathrm{PH}$ may be difficult to identify in patients with respiratory disorders. In addition, in patients with lung disease, peripheral oedema may not necessarily indicate RV failure, as it may also result from the effects of hypoxaemia and hypercapnia on the renin-angiotensin-aldosterone system. Furthermore, concomitant LHD, which is frequently found in patients with chronic respiratory diseases, may also contribute to $\mathrm{PH}$. As a general rule, patients presenting with symptoms that are more severe than expected based on their PFT results should be further evaluated, in particular by echocardiography, to search for concomitant LHD or PH.

Echocardiography remains the most widely used non-invasive diagnostic tool for the assessment of PH. Indications for echocardiography in patients with lung diseases include the clinical suspicion of significant $\mathrm{PH}$ or the evaluation of concomitant LHD. It should be noted, however, that the accuracy of echocardiography in patients with advanced respiratory diseases is low [403-405]. Patients with clinical or echocardiographic signs of severe $\mathrm{PH}$ and/or severe RV dysfunction should be referred to a $\mathrm{PH}$ centre.

A definite diagnosis of $\mathrm{PH}$ relies on measurements obtained during RHC. Potential indications for RHC in advanced lung disease are (i) proper diagnosis or exclusion of $\mathrm{PH}$ in candidates for surgical treatments (transplantation, lung volume reduction), (ii) suspected PAH or CTEPH, (iii) episodes of RV failure and (iv) inconclusive echocardiographic findings in cases with a high level of suspicion and potential therapeutic implications. 


\subsection{Therapy}

Currently there is no specific therapy for $\mathrm{PH}$ associated with lung diseases. Long-term $\mathrm{O}_{2}$ administration has been shown to partially reduce the progression of $\mathrm{PH}$ in COPD. Nevertheless, PAP rarely returns to normal values and the structural abnormalities of pulmonary vessels remain unaltered [169]. In interstitial lung diseases, the role of long-term $\mathrm{O}_{2}$ therapy on $\mathrm{PH}$ progression is less clear.

Treatment with conventional vasodilators such as CCBs is not recommended because they may impair gas exchange due to the inhibition of hypoxic pulmonary vasoconstriction [406-408] and because of their lack of efficacy after long-term use [409, 410].

Published experience with targeted PAH drug therapy is scarce, and so far there is no evidence from RCTs suggesting that PAH drugs result in improved symptoms or outcomes in patients with lung disease [411-416].

In summary, patients with lung disease and $\mathrm{PH}$ who are hypoxaemic should receive long-term $\mathrm{O}_{2}$ therapy, adapting the general recommendations for COPD. Treatment of the underlying lung disease should be optimized. The use of drugs approved for PAH is not recommended for patients with $\mathrm{PH}$ due to lung disease. Patients with suspected PAH in addition to their lung diseases (characterized by mild lung parenchymal abnormalities, symptoms insufficiently explained by lung mechanical disturbances and a haemodynamic 'PAH phenotype', i.e. severe PH with high PVR and low $\mathrm{CO}$ ) may be treated according to the recommendations for $\mathrm{PAH}$, keeping in mind the potential implications of the co-existing lung disease on symptoms and response to therapy.

The recommendations for $\mathrm{PH}$ due to lung diseases are reported in the Table 33.

\section{Chronic thromboembolic pulmonary hypertension (group 4)}

CTEPH is a disease of obstructive PA remodelling as a consequence of major vessel thromboembolism. CTEPH has been reported with a cumulative incidence of $0.1-9.1 \%$ within the first 2 years after a symptomatic PE event [417]. The large margin of error is probably due to referral bias, a paucity of early symptoms and difficulty in differentiating acute PE from symptoms of pre-existing CTEPH [418]. Although the exact prevalence and annual incidence of CTEPH are unknown, some data suggest that this condition may occur in approximately 5 individuals per million population per year [419].

In the differential diagnosis of CTEPH the following conditions have to be considered and treated according to current best knowledge. These conditions include PA sarcoma, tumour cell embolism, parasites (hydatid cyst), foreign body embolism and congenital or acquired PA stenosis (Table 4).

\subsection{Diagnosis}

Routine screening for CTEPH after PE is not supported by current evidence; a significant number of CTEPH cases develop in the absence of previous acute PE.

The median age of patients at diagnosis is 63 years and both genders are equally affected [25]; paediatric cases are rare $[287,420]$. Clinical symptoms and signs are non-specific or absent in early CTEPH, with signs of right heart failure only becoming evident in advanced disease. Thus early diagnosis remains a challenge in CTEPH, with a median time of 14 months between symptom onset and diagnosis in expert centres [421]. When present, the clinical symptoms of CTEPH may resemble those of acute PE or IPAH; in the latter context, oedema and haemoptysis occur more often in CTEPH, while syncope is more common in IPAH [422].

The diagnosis of CTEPH is based on findings obtained after at least 3 months of effective anticoagulation in order to discriminate this condition from 'subacute' PE. These findings are mean PAP $\geqslant 25 \mathrm{mmHg}$ with PAWP $\leqslant 15 \mathrm{mmHg}$, mismatched perfusion defects on lung scan and specific diagnostic signs for CTEPH seen by multidetector CT angiography, MR imaging or conventional pulmonary cineangiography, such as ring-like stenoses, webs/slits and chronic total occlusions (pouch lesions or tapered lesions).

Some patients, particularly those with complete unilateral obstruction, may present with normal pulmonary haemodynamics at rest despite symptomatic disease. These patients are also considered to have CTEPH and are managed accordingly. A suitable terminology to describe this condition of chronic thromboembolic pulmonary vascular disease is still lacking.

An algorithm for CTEPH diagnosis is shown in Figure 3. While CT pulmonary angiography is the investigation of choice for the diagnosis of acute $\mathrm{PE}$, planar V/Q lung scan remains the main first-line imaging modality for CTEPH, as it carries a $96-97 \%$ sensitivity and a $90-95 \%$ specificity for the diagnosis [47]. In contrast, in IPAH and PVOD, perfusion scans typically show non-segmental defects or are normal [377]. More recent work suggests that both V/Q scanning and modern CT pulmonary angiography may be accurate methods for the detection of CTEPH, with excellent diagnostic efficacy in expert hands (100\% sensitivity, 93.7\% specificity and $96.5 \%$ accuracy for V/Q and $96.1 \%, 95.2 \%$ and $95.6 \%$, respectively, for CT pulmonary angiography) $[93,423,424]$. 
TABLE 33 Recommendations for pulmonary hypertension due to lung diseases

\begin{tabular}{|c|c|c|c|}
\hline Recommendations & Class ${ }^{a}$ & Levelb & Ref.c \\
\hline $\begin{array}{l}\text { Echocardiography is recommended } \\
\text { for the non-invasive diagnostic } \\
\text { assessment of suspected } \mathrm{PH} \text { in } \\
\text { patients with lung disease }\end{array}$ & 1 & C & {$[403,405]$} \\
\hline $\begin{array}{l}\text { Referral to an expert centre is } \\
\text { recommendedd in patients with } \\
\text { echocardiographic signs of severe } \\
\mathrm{PH} \text { and/or severe right ventricular } \\
\text { dysfunction }\end{array}$ & 1 & C & \\
\hline $\begin{array}{l}\text { The optimal treatment of the } \\
\text { underlying lung disease, including } \\
\text { long-term } \mathrm{O}_{2} \text { therapy in patients } \\
\text { with chronic hypoxaemia, is } \\
\text { recommended in patients with } \mathrm{PH} \\
\text { due to lung diseases }\end{array}$ & 1 & C & [169] \\
\hline $\begin{array}{l}\text { Referral to } \mathrm{PH} \text { expert center should } \\
\text { be considered for patients with signs } \\
\text { of severe } \mathrm{PH} / \text { severe RV failure for } \\
\text { individual-based treatment }\end{array}$ & Ila & C & \\
\hline $\begin{array}{l}\text { RHC is not recommended for } \\
\text { suspected } \mathrm{PH} \text { in patients with lung } \\
\text { disease, unless therapeutic } \\
\text { consequences are to be expected } \\
\text { (e.g. lung transplantation, } \\
\text { alternative diagnoses such as PAH } \\
\text { or CTEPH, potential enrolment in a } \\
\text { clinical trial) }\end{array}$ & III & C & [169] \\
\hline $\begin{array}{l}\text { The use of drugs approved for } \mathrm{PAH} \text { is } \\
\text { not recommended in patients with } \\
\mathrm{PH} \text { due to lung diseases }\end{array}$ & III & C & [411-416] \\
\hline
\end{tabular}

CTEPH: chronic thromboembolic pulmonary hypertension; $\mathrm{PAH}$ : pulmonary arterial hypertension; $\mathrm{PH}$ : pulmonary hypertension; RHC: right heart catheterization. ${ }^{a}$ Class of recommendation. ${ }^{\text {b }}$ evel

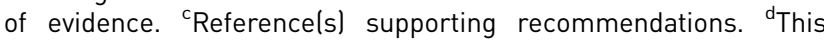
recommendation does not apply to patients with end-stage lung disease who are not considered candidates for lung transplantation.

Multidetector CT pulmonary angiography has become an established imaging modality for confirming CTEPH [93], however, this investigation alone cannot exclude the disease [47]. CT pulmonary angiography may help to identify complications of the disease such as PA dilatation resulting in left main coronary artery compression and hypertrophied bronchial arterial collaterals, which may lead to haemoptysis.

A high-resolution CT scan of the chest delivers images of the lung parenchyma and identifies emphysema, bronchial disease or interstitial lung disease, as well as infarcts, vascular and pericardial malformations and thoracic wall deformities. Perfusion inequalities manifest as a mosaic parenchymal pattern, with dark areas corresponding to relatively decreased perfusion. Although a mosaic pattern is frequent in CTEPH, it can also be observed in up to $12 \%$ of patients with PAH [425]. MR imaging of the pulmonary vasculature is still considered inferior to CT [426], but this modality, as well as cone beam CT [427], angioscopy [428], intravascular ultrasound or optical coherence tomography, may be complimentary and used according to local experience and practice.

RHC is an essential diagnostic tool. Preoperative and immediate postoperative PVR is a long-term predictor of prognosis [429]. The final step in the diagnostic pathway is selective pulmonary angiography in the anterior-posterior and lateral projections illustrating ring-like stenosis, webs ('slits'), pouches, wall irregularities, complete vascular obstructions as well as bronchial collaterals, and supports the technical assessment of operability. 


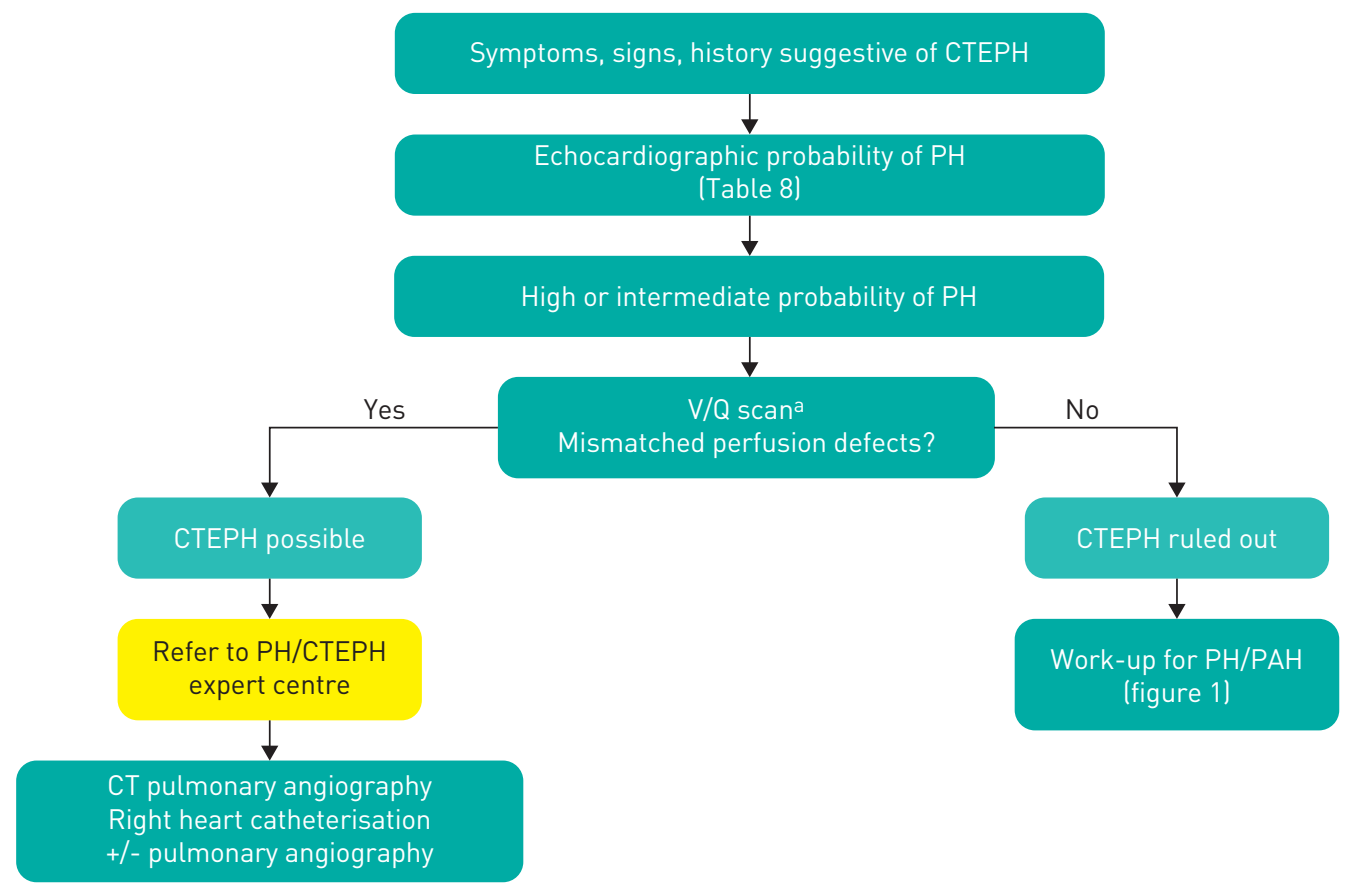

FIGURE 3 Diagnostic algorithm for chronic thromboembolic pulmonary hypertension. CT: computed thomography; CTEPH: chronic thromboembolic pulmonary hypertension; $\mathrm{PAH}$ : pulmonary arterial hypertension; $\mathrm{PH}$ : pulmonary hypertension; $\mathrm{V} / \mathrm{Q}$ : ventilation/perfusion. ${ }^{\mathrm{C}} \mathrm{CT}$ pulmonary angiography alone may miss diagnosis of chronic thromboembolic pulmonary hypertension.

\subsection{Therapy}

10.2.1 Surgical

PEA is the treatment of choice for CTEPH (Figure 4). In Europe, in-hospital mortality is currently as low as $4.7 \%$ [430], and even lower in high-volume single centres [431]. The majority of patients experience substantial relief from symptoms and near normalization of haemodynamics [430-432]. In contrast to surgical embolectomy for acute $\mathrm{PE}$, treatment of $\mathrm{CTEPH}$ necessitates a true bilateral endarterectomy through the medial layer of the pulmonary arteries, which is performed under deep hypothermia and circulatory arrest [431], without the need for cerebral perfusion [433].

Operability of patients with CTEPH is determined by multiple factors that cannot easily be standardized; these are related to the suitability of the patient, the expertise of the surgical team and available resources. General criteria include preoperative WHO-FC II-IV and surgical accessibility of thrombi in the main, lobar or segmental pulmonary arteries. Advanced age per se is not a contraindication for surgery. There is no PVR threshold or measure of RV dysfunction that can be considered to preclude PEA.

Postoperative ECMO is recommended as a standard of care in PEA centres for severe cases [434-436]. Early postoperative reperfusion oedema may require veno-arterial ECMO, and severe persistent $\mathrm{PH}$ may be bridged to emergency lung transplantation with veno-venous ECMO.

Patients who do not undergo PEA or suffer from persistent or recurrent PH after PEA (post-PEA PH) face a poor prognosis.

\subsubsection{Medical}

Optimal medical treatment for CTEPH consists of anticoagulants and diuretics, and $\mathrm{O}_{2}$ in cases of heart failure or hypoxaemia. Lifelong anticoagulation is recommended, even after PEA, though no data exist on the efficacy and safety of new oral anticoagulants. Although there is no consensus, routine cava filter placement is not justified by the available evidence. Pulmonary microvascular disease in CTEPH has provided the rationale for off-label use of drugs approved for PAH [25]. Some non-randomized studies have provided evidence for improvement in exercise capacity and haemodynamics [437-439]. Medical treatment of CTEPH with targeted therapy may be justified in technically non-operable patients or in the presence of an unacceptable surgical risk:benefit ratio (Figure 2). Patients with persistent or recurrent PH after PEA may also be candidates for targeted medical therapy. The use of targeted therapy in operable patients with severe haemodynamic compromise as a bridge to PEA has not yet been supported by scientific evidence. 


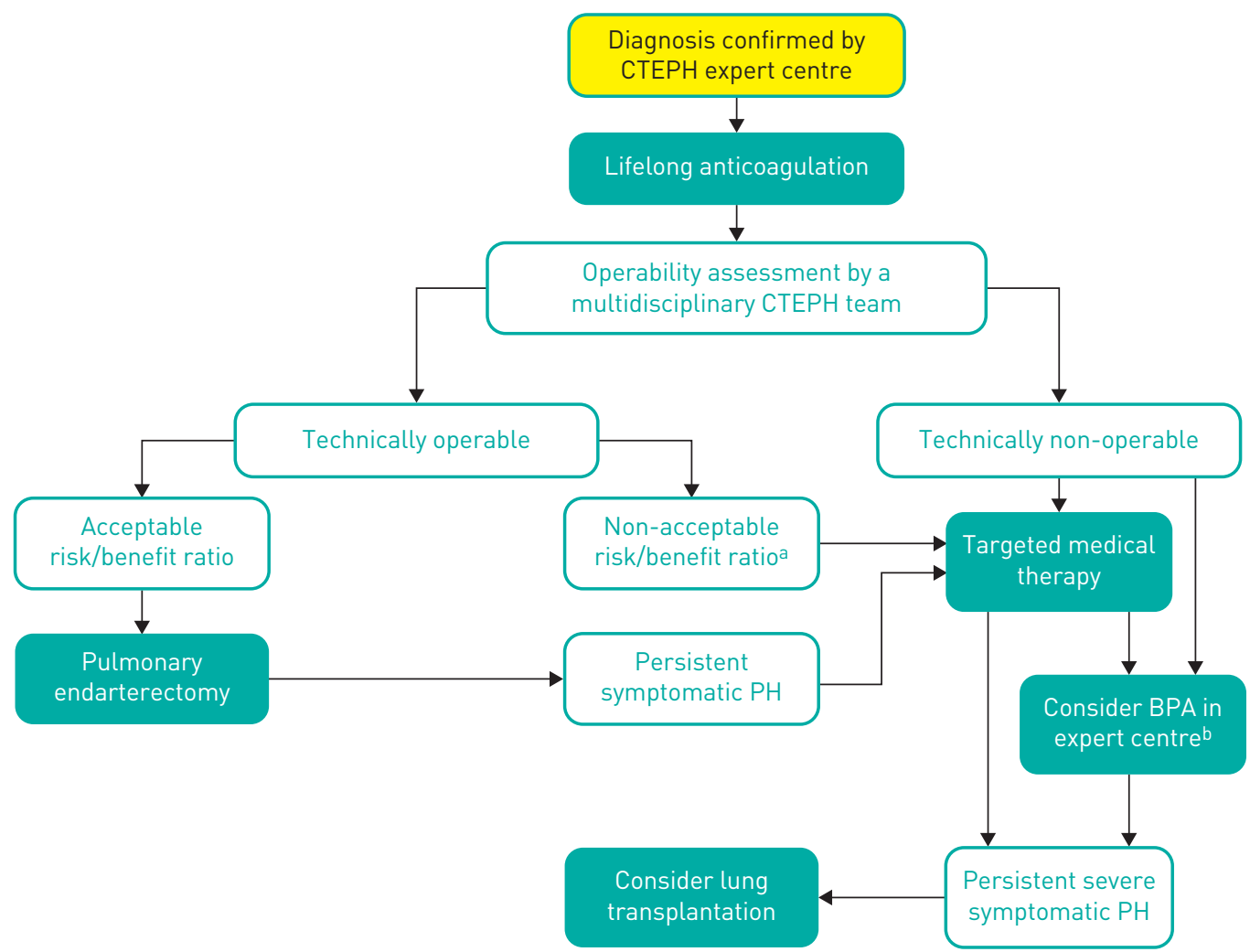

FIGURE 4 Treatment algorithm for chronic thromboembolic pulmonary hypertension. BPA: balloon pulmonary angioplasty; CTEPH: chronic thromboembolic pulmonary hypertension; $\mathrm{PH}$ : pulmonary hypertension. ${ }^{\mathrm{a} T e c h n i c a l l y}$ operable patients with non-acceptable risk/benefit ratio can be considered also for BPA. ${ }^{\mathrm{b}}$ In some centers medical therapy and BPA are initiated concurrently.

The dual endothelin antagonist bosentan was evaluated in 157 patients with inoperable CTEPH or persistent/recurrent PH after PEA over 16 weeks; the primary combined endpoint of a decrease in PVR and an increase in the 6MWD was not met [440]. However, an oral sGC stimulator, riociguat, was administered to 261 of 446 screened patients with non-operable CTEPH or persistent/recurrent $\mathrm{PH}$ after PEA for 16 weeks and led to a mean increase of $39 \mathrm{~m}$ in the 6MWD $(P<0.001$, primary endpoint) and to a least squares mean difference of $246 \mathrm{dyn} \cdot \mathrm{cm} \cdot \mathrm{s}^{-5}$ in PVR $(P<0.001$, secondary endpoint); the time to clinical worsening remained unchanged [441].

Preoperative medical treatment is uncertain because the magnitude of effects was small in one RCT [442]. One retrospective study indicated no difference in outcome, but there was a delay in surgery for patients treated medically [442]. Prospective RCTs are needed in patients with potential treatment benefit; for example, patients with a high PVR and technically challenging anatomy.

After PEA, patients should be followed in CTEPH centres, with at least one haemodynamic assessment to be considered at 6-12 months after the intervention.

\subsubsection{Interventional}

In 2001 Feinstein et al. [443]. published a series of 18 patients with non-operable CTEPH who had been subjected to balloon dilatation of the pulmonary arteries. Despite a significant decrease in PAPm, 11 patients developed reperfusion pulmonary oedema and 3 required mechanical ventilation. Recently, Japanese investigators have refined BPA by using smaller balloons, by cautiously limiting the number of balloon inflations per session to one or two pulmonary vascular segments and by the use of intravascular imaging [444-446]. An average number of 4.8 sessions is needed per patient to improve parameters of RV function [57]. A careful approach with targeting only one lobe during each session and very cautious balloon sizing have reduced the incidence of reperfusion pulmonary oedema to $2 \%$ in individual centres [447]. While BPA is still not extensively used, [448]. it is rapidly gaining attention worldwide. BPA should only be performed in experienced and high-volume CTEPH centres.

The recommendations for CTEPH are summarised in Table 34. 
An algorithm for the treatment of CTEPH is provided in Figure 4.

\section{Pulmonary hypertension with unclear and/or multifactorial mechanisms (group 5)}

$\mathrm{PH}$ with unclear and/or multifactorial mechanisms (group 5, Table 4) includes several disorders with multiple patho-aetiologies. A common feature of these diseases is that the mechanisms of $\mathrm{PH}$ are poorly understood and may include pulmonary vasoconstriction, proliferative vasculopathy, extrinsic compression, intrinsic occlusion, high-output cardiac failure, vascular obliteration and left heart failure as causes (Web Table VIII).

These patients need careful diagnosis. Treatment is tailored for that diagnosis; treatment of $\mathrm{PH}$ is secondary. The axiom should be 'Treat the lung not the pressure'. There are no RCTs regarding the use of $\mathrm{PAH}$-approved drugs in the treatment of group 5 disorders [450]. Of particular importance is that some of the diseases described in Web Table VII may have a venous component (PVOD) that could be made worse by the use of pulmonary arterial vasodilators.

\section{Definition of a pulmonary hypertension referral centre}

$\mathrm{PAH}$ is a rare disease. Since, in general, medical centres with a high volume of patients tend to obtain the best outcomes, the establishment of expert referral centres is clinically and economically highly desirable and supported by patient organisations. The purpose of a referral centre is to receive new referrals and undertake assessment and investigation of all causes of $\mathrm{PH}$, routinely manage appropriate patients with $\mathrm{PAH}$ - and CTEPH-specific drug therapies, work closely with other healthcare providers to obtain the best outcomes for patients and undertake audits, research and education.

Referral centres should have sufficient patients on chronic therapy as well as new referrals to warrant this status. The ideal number of patients seen by an adult centre each year is recommended to be no fewer than 200, of which at least half have a final diagnosis of PAH. In countries with a population $>10$ million, adult centres should ideally expand to accommodate $>300$ patients annually. It is recommended that a referral centre, as a minimum, should follow at least 50 patients with PAH or CTEPH and receive at least two new referrals per month with documented PAH or CTEPH. Paediatric centres are recommended to see 30-50 patients per year. These numbers can be adapted according to specific country characteristics (population distribution, geographical constraints, etc.).

\subsection{Facilities and skills required for a referral centre}

1. Referral centres are recommended to provide care by an interprofessional team that should, as a minimum, comprise [451-456]:

(a) two consultant physicians (normally from either or both cardiology and respiratory medicine) experienced in and with a special interest in $\mathrm{PH}$ with dedicated $\mathrm{PH}$ clinical sessions for outpatients, inpatients and a multidisciplinary team meeting

(b) clinical nurse specialist

(c) radiologist with expertise in pulmonary hypertension imaging

(d) cardiologist or $\mathrm{PH}$ physician with expertise in echocardiography

(e) cardiologist or $\mathrm{PH}$ physician with expertise in RHC and vasoreactivity testing

(f) access to psychological and social work support

(g) appropriate on-call cover and expertise

2. For referral centres, access to the following facilities is recommended:

(a) a ward where staff has special expertise in $\mathrm{PH}$

(b) an intensive therapy unit with relevant expertise

(c) a specialist outpatient service

(d) emergency care

(e) diagnostic investigations including echocardiography, CT scanning, nuclear scanning, MR imaging, ultrasound, exercise testing, lung function testing and a cardiac catheterization laboratory

(f) access to the full range of specific PAH and CTEPH drug therapy available in their country

3. Referral centres are recommended to have established networks (e.g. referral criteria, patient pathway and clinical management protocols) with other services that may not necessarily be on the same site [452]:
(a) genetics
(b) CTD
(c) family planning
(d) PEA
(e) lung transplantation
(f) adult $\mathrm{CHD}$ 
TABLE 34 Recommendations for chronic thromboembolic pulmonary hypertension

\begin{tabular}{|c|c|c|c|}
\hline Recommendations & Class ${ }^{a}$ & Level $^{b}$ & Ref.c \\
\hline $\begin{array}{l}\text { In PE survivors with exercise dyspnoea, } \\
\text { CTEPH should be considered }\end{array}$ & Ila & C & [449] \\
\hline $\begin{array}{l}\text { Life-long anticoagulation is } \\
\text { recommended in all patients with } \\
\text { CTEPH }\end{array}$ & I & C & [91] \\
\hline $\begin{array}{l}\text { It is recommended that in all patients } \\
\text { with CTEPH the assessment of } \\
\text { operability and decisions regarding } \\
\text { other treatment strategies should be } \\
\text { made by a multidisciplinary team of } \\
\text { experts }\end{array}$ & 1 & C & [91] \\
\hline $\begin{array}{l}\text { Surgical PEA in deep hypothermia } \\
\text { circulatory arrest is recommended for } \\
\text { patients with CTEPH }\end{array}$ & I & C & [91] \\
\hline $\begin{array}{l}\text { Riociguat is recommended in } \\
\text { symptomatic patients who have been } \\
\text { classified as having } \\
\text { persistent/recurrent CTEPH after } \\
\text { surgical treatment or inoperable } \\
\text { CTEPH by a CTEPH team including at } \\
\text { least one experienced PEA surgeon }\end{array}$ & I & B & [441] \\
\hline $\begin{array}{l}\text { Off-label use of drugs approved for PAH } \\
\text { may be considered in symptomatic } \\
\text { patients who have been classified as } \\
\text { having inoperable CTEPH by a CTEPH } \\
\text { team including at least one } \\
\text { experienced PEA surgeon }\end{array}$ & IIb & B & [437-440] \\
\hline $\begin{array}{l}\text { Interventional BPA may be considered } \\
\text { in patients who are technically } \\
\text { non-operable or carry an unfavourable } \\
\text { risk:benefit ratio for PEA }\end{array}$ & IIb & C & $\begin{array}{c}{[57} \\
444-446 \\
448]\end{array}$ \\
\hline $\begin{array}{l}\text { Screening for CTEPH in asymptomatic } \\
\text { survivors of PE is currently not } \\
\text { recommended }\end{array}$ & III & C & [417] \\
\hline
\end{tabular}

BPA: balloon pulmonary angioplasty; CTEPH: chronic thromboembolic pulmonary hypertension; $\mathrm{PAH}$ : pulmonary arterial hypertension; $\mathrm{PE}$ : pulmonary embolism; PEA: pulmonary endarterectomy. ${ }^{a}$ Class of recommendation. ${ }^{\mathrm{b}}$ Level of evidence. ${ }^{\mathrm{c}}$ Referencels) supporting recommendations.
TABLE 35 Recommendations for pulmonary hypertension referral centres

\begin{tabular}{|l|c|c|}
\hline Recommendations & Class $^{a}$ & Level $^{b}$ \\
\hline $\begin{array}{l}\text { It is recommended for referral centres to provide } \\
\text { care by a multiprofessional team (cardiology and } \\
\text { respiratory medicine physicians, clinical nurse } \\
\text { specialist, radiologists, psychological and social } \\
\text { work support, appropriate on-call expertise) }\end{array}$ & I & C \\
\hline $\begin{array}{l}\text { It is recommended for referral centres to have } \\
\text { direct links and quick referral patterns to other } \\
\text { services (such as CTD, family planning, PEA, lung } \\
\text { transplantation, adult congenital heart disease) }\end{array}$ & I & C \\
\hline $\begin{array}{l}\text { It should be considered that a referral centre follow } \\
\text { at least } 50 \text { patients with PAH or CTEPH and should } \\
\text { receive at least two new referrals per month with } \\
\text { documented PAH or CTEPH }\end{array}$ & Ila & $\mathrm{C}$ \\
\hline $\begin{array}{l}\text { It should be considered that a referral centre } \\
\text { perform at least } 20 \text { vasoreactivity tests in IPAH, } \\
\text { HPAH or DPAH patients per year }\end{array}$ & Ila & $\mathrm{C}$ \\
\hline $\begin{array}{l}\text { Referral centres should participate in collaborative } \\
\text { clinical research in PAH, including phase II and } \\
\text { phase III clinical trials }\end{array}$ & Ila & $\mathrm{C}$ \\
\hline
\end{tabular}

CTD: connective tissue disease; CTEPH: chronic thromboembolic pulmonary hypertension; DPAH: drug-induced pulmonary arterial hypertension; HPAH: heritable pulmonary arterial hypertension; IPAH: idiopathic pulmonary arterial hypertension; PAH: pulmonary arterial hypertension; PEA: pulmonary endarterectomy. ${ }^{a}$ Class of recommendation. ${ }^{b}$ Level of evidence. ${ }^{\text {CReference(s) supporting }}$ recommendations.

4. Referral centres should consider undertaking a programme of clinical audit of adherence to guidelines and clinical outcomes that includes survival analysis. Audits should also carry out comparisons within the same country where there is more than one referral centre.

5. Referral centres should consider participating in collaborative clinical research in PAH and CTEPH that includes phase II and phase III clinical trials.

6. Referral centres should consider raising awareness about referral criteria and provide regular education about all aspects of $\mathrm{PH}$ to appropriate healthcare professionals. In particular, education should be aimed at junior doctors in training as well as senior colleagues.

7. Referral centres should consider participating in the development and running of a network of $\mathrm{PH}$ centres within their own country where there is more than one referral centre.

8. Referral centres should consider having a link to their national and/or European PH patients' associations. The recommendations for pulmonary hypertension referral centres are reported in the Table 35. 


\section{To do and not to do messages from the guidelines}

\begin{tabular}{|c|c|c|}
\hline \multicolumn{3}{|l|}{ Pulmonary hypertension diagnosis } \\
\hline $\begin{array}{l}\text { Right heart catheterization is recommended to confirm the } \\
\text { diagnosis of pulmonary arterial hypertension (PAH - } \\
\text { Group 1) and to support treatment decisions }\end{array}$ & I & C \\
\hline $\begin{array}{l}\text { Vasoreactivity testing is recommended in patients with } \\
\text { IPAH, HPAH and PAH induced by drugs use to detect } \\
\text { patients who can be treated with high doses of a calcium } \\
\text { channel blocker }\end{array}$ & I & C \\
\hline \multicolumn{3}{|l|}{ Pulmonary arterial hypertension severity } \\
\hline $\begin{array}{l}\text { It is recommended to evaluate the severity of PAH patients } \\
\text { with a panel of data derived from clinical assessment, } \\
\text { exercise tests, biochemical markers, and } \\
\text { echocardiographic and haemodynamic evaluation (Tables } \\
13 \text { and } 14 \text { ) and to perform regular follow-up assessments } \\
\text { every 3-6 months in stable patients (Table 14) }\end{array}$ & 1 & C \\
\hline \multicolumn{3}{|l|}{ Pulmonary arterial hypertension general measures } \\
\hline It is recommended to avoid pregnancy in patients with $\mathrm{PAH}$ & । & C \\
\hline \multicolumn{3}{|l|}{ Pulmonary arterial hypertension therapy } \\
\hline $\begin{array}{l}\text { It is recommended for referral centres to provide care by a } \\
\text { multi-professional team (cardiology and respiratory } \\
\text { medicine physicians, clinical nurse specialist, } \\
\text { radiologists, psychological and social work support, } \\
\text { appropriate on-call expertise) }\end{array}$ & I & C \\
\hline $\begin{array}{l}\text { Initial approved drugs monotherapy is recommended in } \\
\text { treatment naïve, low or intermediate risk patients with } \\
\text { pulmonary arterial hypertension (Table 19) }\end{array}$ & I & A \\
\hline $\begin{array}{l}\text { Initial approved oral drugs combination therapy is } \\
\text { recommended in treatment naïve, low or intermediate } \\
\text { risk patients with pulmonary arterial hypertension (Table } \\
\text { 20) }\end{array}$ & I & B \\
\hline $\begin{array}{l}\text { Sequential drugs combination therapy is recommended in } \\
\text { patients with inadequate treatment response to initial } \\
\text { monotherapy or to initial double combination therapy } \\
\text { (Table 21) }\end{array}$ & I & B \\
\hline \multicolumn{3}{|l|}{ Recommendations for left heart disease and lung diseases } \\
\hline $\begin{array}{l}\text { The use of PAH approved therapies is not recommended in } \\
\text { patients with pulmonary hypertension due to left heart } \\
\text { disease or lung diseases }\end{array}$ & III & C \\
\hline \multicolumn{3}{|l|}{$\begin{array}{l}\text { Recommendations for chronic thromboembolic pulmonary } \\
\text { hypertension }\end{array}$} \\
\hline $\begin{array}{l}\text { Surgical pulmonary endarterectomy in deep hypothermia } \\
\text { circulatory arrest is recommended for patients with } \\
\text { CTEPH and it is recommended that the assessment of } \\
\text { operability and decisions regarding other treatment } \\
\text { strategies (drugs therapy or balloon pulmonary } \\
\text { angioplasty) be made by a multidisciplinary team of } \\
\text { experts }\end{array}$ & 1 & C \\
\hline
\end{tabular}

\section{Appendix}

Task Force Members: Nazzareno Galiè (ESC Chairperson) (Italy), Marc Humbert (ERS Chairperson) (France), Jean-Luc Vachiery (Belgium), Simon Gibbs (UK), Irene Lang (Austria), Adam Torbicki (Poland), Gérald Simonneau (France), Andrew Peacock (UK), Anton Vonk Noordegraaf (The Netherlands), Maurice 
Beghetti (Switzerland), Ardeschir Ghofrani (Germany), Miguel Angel Gomez Sanchez (Spain), Georg Hansmann (Germany), Walter Klepetko (Austria), Patrizio Lancellotti (Belgium), Marco Matucci (Italy), Theresa McDonagh (UK), Luc A. Pierard (Belgium), Pedro T. Trindade (Switzerland), Maurizio Zompatori (Italy) and Marius Hoeper (Germany). Document reviewers: Victor Aboyans (CPG Review Coordinator) (France), Antonio Vaz Carneiro (CPG Review Coordinator) (Portugal), Stephan Achenbach (Germany), Stefan Agewall (Norway), Yannick Allanore (France), Riccardo Asteggiano (Italy), Luigi Paolo Badano (Italy), Joan Albert Barberà (Spain), Hélène Bouvaist (France), Héctor Bueno (Spain), Robert A. Byrne (Germany), Scipione Carerj (Italy), Gra̧̧a Castro (Portugal), Çetin Erol (Turkey), Volkmar Falk (Germany), Christian Funck-Brentano (France), Matthias Gorenflo (Germany), John Granton (Canada), Bernard Iung (France), David G. Kiely (UK), Paulus Kirchhof (Germany/UK), Barbro Kjellstrom (Sweden), Ulf Landmesser (Switzerland), John Lekakis (Greece), Christos Lionis (Greece), Gregory Y.H. Lip (UK), Stylianos E. Orfanos (Greece), Myung H. Park (USA), Massimo F. Piepoli (Italy), Piotr Ponikowski (Poland), Marie-Pierre Revel (France), David Rigau (ERS methodologist) (Switzerland), Stephan Rosenkranz (Germany), Heinz Völler (Germany), and Jose Luis Zamorano (Spain).

ESC Associations: Acute Cardiovascular Care Association (ACCA), European Association for Cardiovascular Prevention and Rehabilitation (EACPR), European Association of Cardiovascular Imaging (EACVI), European Association of Percutaneous Cardiovascular Interventions (EAPCI), European Heart Rhythm Association (EHRA), Heart Failure Association (HFA).

ESC Councils: Council for Cardiology Practice (CCP), Council on Cardiovascular Nursing and Allied Professions (CCNAP), Council on Cardiovascular Primary Care (CCPC).

ESC Working Groups: Cardiovascular Pharmacotherapy, Cardiovascular Surgery, Grown-up Congenital Heart Disease, Pulmonary Circulation and Right Ventricular Function, Valvular Heart Disease.

ESC Committee for Practice Guidelines (CPG): Jose Luis Zamorano (Chairperson) (Spain), Victor Aboyans (France), Stephan Achenbach (Germany), Stefan Agewall (Norway), Lina Badimon (Spain), Gonzalo Barón-Esquivias (Spain), Helmut Baumgartner (Germany), Jeroen J. Bax (The Netherlands), Héctor Bueno (Spain), Scipione Carerj (Italy), Veronica Dean (France), Çetin Erol (Turkey), Donna Fitzsimons (UK), Oliver Gaemperli (Switzerland), Paulus Kirchhof (Germany/UK), Philippe Kolh (Belgium), Patrizio Lancellotti (Belgium), Gregory Y.H. Lip (UK), Petros Nihoyannopoulos (UK), Massimo F. Piepoli (Italy), Piotr Ponikowski (Poland), Marco Roffi (Switzerland), Adam Torbicki (Poland), Antonio Vaz Carneiro (Portugal), Stephan Windecker (Switzerland).

ESC National Cardiac Societies actively involved in the review process of the 2015 ESC/ERS Guidelines for the diagnosis and treatment of pulmonary hypertension:

Albania: Albanian Society of Cardiology, Sokol Myftiu; Austria: Austrian Society of Cardiology, Diana Bonderman; Azerbaijan: Azerbaijan Society of Cardiology, Ibrahimov Firdovsi; Belarus: Belorussian Scientific Society of Cardiologists, Irina Lazareva; Belgium: Belgian Society of Cardiology, Michel De Pauw; Bosnia \& Herzegovina: Association of Cardiologists of Bosnia \& Herzegovina, Šekib Sokolović; Bulgaria: Bulgarian Society of Cardiology, Vasil Velchev; Croatia: Croatian Cardiac Society, Maja Čikeš; Cyprus: Cyprus Society of Cardiology, Josef Antoniou Moutiris; Czech Republic: Czech Society of Cardiology, Pavel Jansa; Denmark: Danish Society of Cardiology, Jens Erik Nielsen-Kudsk; Estonia: Estonian Society of Cardiology, Ly Anton; Finland: Finnish Cardiac Society, Pertti Jääskeläinen; France: French Society of Cardiology, Fabrice Bauer; Georgia: Georgian Society of Cardiology, Archil Chukhrukidze; Germany: German Cardiac Society, Christian Opitz; Greece: Hellenic Cardiological Society, George Giannakoulas; Hungary: Hungarian Society of Cardiology, Kristóf Karlócai; Iceland: Icelandic Society of Cardiology, Hjörtur Oddsson; Ireland: Irish Heart Foundation, Sean Gaine; Israel: Israel Heart Society, Doron Menachemi; Italy: Italian Federation of Cardiology, Michele Emdin; Kyrgyzstan: Kyrgyz Society of Cardiology, Talant Sooronbaev; Latvia: Latvian Society of Cardiology, Ainars Rudzitis; Lithuania: Lithuanian Society of Cardiology, Lina Gumbiene; Luxembourg: Luxembourg Society of Cardiology, Frederic Lebrun; Malta: Maltese Cardiac Society, Josef Micallef; Moldavia: Moldavian Society of Cardiology, Victor Botnaru; Morocco: Moroccan Society of Cardiology, Latifa Oukerraj; Norway: Norwegian Society of Cardiology, Arne K. Andreassen; Poland: Polish Cardiac Society, Marcin Kurzyna; Portugal: Portuguese Society of Cardiology, Maria João Ribeiro Leite Baptista; Romania: Romanian Society of Cardiology, Ioan Mircea Coman; Russia: Russian Society of Cardiology, Olga Moiseeva; Serbia: Cardiology Society of Serbia, Branislav S. Stefanović; Slovakia: Slovak Society of Cardiology, Iveta Šimková; Sweden: Swedish Society of Cardiology, Gerhard Wikström; Switzerland: Swiss Society of Cardiology, Markus Schwerzmann; The Former Yugoslav Republic of Macedonia: Macedonian FYR Society of Cardiology, Elizabeta Srbinovska-Kostovska; The Netherlands: Netherlands Society of Cardiology, Arie P. J. van Dijk; Tunisia: Tunisian Society of Cardiology and Cardio-Vascular Surgery, Abdallah Mahdhaoui; Turkey: Turkish Society of Cardiology, Cihangir Kaymaz; UK: British Cardiovascular Society, Gerry Coghlan; Ukraine: Ukrainian Association of Cardiology, Yuriy Sirenko. 
15. Web addenda

This article has supplementary materials available from erj.ersjournals.com

16. References

Hoeper MM, Bogaard HJ, Condliffe R, et al. Definitions and diagnosis of pulmonary hypertension. J Am Coll Cardiol 2013; 62(Suppl):D42-D50.

2 Kovacs G, Berghold A, Scheidl S, et al. Pulmonary arterial pressure during rest and exercise in healthy subjects A systematic review. Eur Respir J 2009; 34: 888-894.

3 Herve P, Lau E, Sitbon O, et al. Criteria for diagnosis of exercise pulmonary hypertension. Eur Respir J 2015; 46: 728-737.

4 Vachiery JL, Adir Y, Barbera JA, et al. Pulmonary hypertension due to left heart disease. J Am Coll Cardiol 2013; 62: D100-D108.

5 Simonneau G, Galiè N, Rubin LJ, et al. Clinical classification of pulmonary hypertension. J Am Coll Cardiol 2004;43(Suppl 1):S5-S12.

6 Simonneau G, Gatzoulis MA, Adatia I, et al. Updated clinical classification of pulmonary hypertension. $\mathrm{J} \mathrm{Am}$ Coll Cardiol 2013; 62: D34-D41.

7 Dhillon R. The management of neonatal pulmonary hypertension. Arch Dis Child Fetal Neonatal Ed 2012; 97: F223-F228.

8 Porta NF, Steinhorn RH. Pulmonary vasodilator therapy in the NICU: inhaled nitric oxide, sildenafil, and other pulmonary vasodilating agents. Clin Perinatol 2012; 39: 149-164.

9 Ivy DD, Abman SH, Barst RJ, et al. Pediatric pulmonary hypertension. J Am Coll Cardiol 2013; 62: D117-D126.

10 Humbert M, Sitbon O, Chaouat A, et al. Pulmonary arterial hypertension in France: results from a national registry. Am J Respir Crit Care Med 2006; 173: 1023-1030.

11 Peacock AJ, Murphy NF, McMurray JJV, et al. An epidemiological study of pulmonary arterial hypertension. Eur Respir J 2007; 30: 104-109.

12 McGoon MD, Benza RL, Escribano-Subias P, et al. Pulmonary arterial hypertension: epidemiology and registries. J Am Coll Cardiol 2013;62(Suppl):D51-D59.

13 Simonneau G, Robbins I, Beghetti M, et al. Updated clinical classification of pulmonary hypertension. J Am Coll Cardiol 2009;54(Suppl):S43-S54.

14 Montani D, Bergot E, Günther S, et al. Pulmonary arterial hypertension in patients treated by dasatinib. Circulation 2012; 125: 2128-2137.

15 Savale L, Chaumais MC, Cottin V, et al. Pulmonary hypertension associated with benfluorex exposure. Eur Respir J 2012; 40: 1164-1172.

16 Savale L, Sattler C, Gunther S, et al. Pulmonary arterial hypertension in patients treated with interferon. Eur Respir J 2014; 44: 1627-1634.

17 Badesch BD, Champion HC, Gomez-Sanchez MA, et al. Diagnosis and assessment of pulmonary arterial hypertension. J Am Coll Cardiol 2009;54(Suppl):S55-S56.

18 Oudiz RJ. Pulmonary hypertension associated with left-sided heart disease. Clin Chest Med 2007; 28: 233-241.

19 Vahanian A, Alfieri O, Andreotti F, et al. Guidelines on the management of valvular heart disease (version 2012). Eur Heart J 2012; 33: 2451-2496.

20 Seeger W, Adir Y, Barberà JA, et al. Pulmonary hypertension in chronic lung diseases. J Am Coll Cardiol 2013;62 (Suppl):D109-D116.

21 Hurdman J, Condliffe R, Elliot CA, et al. Pulmonary hypertension in COPD: results from the ASPIRE registry. Eur Respir J 2013; 41: 1292-1301.

22 Cottin V, Nunes H, Brillet PY, et al. Combined pulmonary fibrosis and emphysema: a distinct underrecognised entity. Eur Respir J 2005; 26: 586-593.

23 Escribano-Subias P, Blanco I, Lopez-Meseguer M, et al. Survival in pulmonary hypertension in Spain: insights from the Spanish registry. Eur Respir J 2012; 40: 596-603.

24 Pengo V, Lensing AW, Prins $\mathrm{MH}$, et al. Incidence of chronic thromboembolic pulmonary hypertension after pulmonary embolism. $N$ Engl J Med 2004; 350: 2257-2264.

25 Pepke-Zaba J, Delcroix M, Lang I, et al. Chronic thromboembolic pulmonary hypertension (CTEPH): results from an international prospective registry. Circulation 2011; 124: 1973-1981.

26 Soubrier F, Chung WK, Machado R, et al. Genetics and genomics of pulmonary arterial hypertension. J Am Coll Cardiol 2013;62(Suppl):D13-D21.

27 Ma L, Roman-Campos D, Austin ED, et al. A novel channelopathy in pulmonary arterial hypertension. $N$ Engl $J$ Med 2013; 369: 351-361.

28 Eyries M, Montani D, Girerd B, et al. EIF2AK4 mutations cause pulmonary veno-occlusive disease, a recessive form of pulmonary hypertension. Nat Genet 2014; 46: 65-69.

29 Eddahibi S, Chaouat A, Morrell N, et al. Polymorphism of the serotonin transporter gene and pulmonary hypertension in chronic obstructive pulmonary disease. Circulation 2003; 108: 1839-1844.

30 Bonderman D, Wexberg P, Martischnig AM, et al. A noninvasive algorithm to exclude pre-capillary pulmonary hypertension. Eur Respir J 2011; 37: 1096-1103.

31 Rich JD, Thenappan T, Freed B, et al. QTc prolongation is associated with impaired right ventricular function and predicts mortality in pulmonary hypertension. Int J Cardiol 2013; 167: 669-676.

32 Sun PY, Jiang X, Gomberg-Maitland M, et al. Prolonged QRS duration: a new predictor of adverse outcome in idiopathic pulmonary arterial hypertension. Chest 2012; 141: 374-380.

33 Olsson KM, Nickel NP, Tongers J, et al. Atrial flutter and fibrillation in patients with pulmonary hypertension. Int J Cardiol 2013; 167: 2300-2305.

34 Rich S, Dantzker DR, Ayres SM, et al. Primary pulmonary hypertension: a national prospective study. Ann Intern Med 1987; 107: 216-223.

35 Milne EN. Forgotten gold in diagnosing pulmonary hypertension: the plain chest radiograph. Radiographics 2012; 32: 1085-1087. 
Trip P, Nossent EJ, de Man FS, et al. Severely reduced diffusion capacity in idiopathic pulmonary arterial hypertension: patient characteristics and treatment responses. Eur Respir J 2013; 42: 1575-1585.

Sun XG, Hansen JE, Oudiz RJ, et al. Pulmonary function in primary pulmonary hypertension. J Am Coll Cardiol 2003; 41: 1028-1035.

Hoeper MM, Pletz MW, Golpon H, et al. Prognostic value of blood gas analyses in patients with idiopathic pulmonary arterial hypertension. Eur Respir J 2007; 29: 944-950.

Pellegrino R, Viegi G, Brusasco V, et al. Interpretative strategies for lung function tests. Eur Respir J 2005; 26: 948-968.

Holverda S, Bogaard HJ, Groepenhoff H, et al. Cardiopulmonary exercise test characteristics in patients with chronic obstructive pulmonary disease and associated pulmonary hypertension. Respiration 2008; 76: $160-167$.

Jilwan FN, Escourrou P, Garcia G, et al. High occurrence of hypoxemic sleep respiratory disorders in precapillary pulmonary hypertension and mechanisms. Chest 2013; 143: 47-55.

Rafanan AL, Golish JA, Dinner DS, et al. Nocturnal hypoxemia is common in primary pulmonary hypertension. Chest 2001; 120: 894-899.

Rudski LG, Lai WW, Afilalo J, et al. Guidelines for the echocardiographic assessment of the right heart in adults: a report from the American Society of Echocardiography endorsed by the European Association of Echocardiography, a registered branch of the European Society of Cardiology, and the Canadian Society of Echocardiography. J Am Soc Echocardiogr 2010; 23: 685-713.

Lang RM, Badano LP, Mor-Avi V, et al. Recommendations for cardiac chamber quantification by echocardiography in adults: an update from the American Society of Echocardiography and the European Association of Cardiovascular Imaging. Eur Heart J Cardiovasc Imaging 2015; 16: 233-271.

Foale R, Nihoyannopoulos P, McKenna W, et al. Echocardiographic measurement of the normal adult right ventricle. Br Heart J 1986; 56: 33-44.

Hachulla E, Gressin V, Guillevin L, et al. Early detection of pulmonary arterial hypertension in systemic sclerosis: a French nationwide prospective multicenter study. Arthritis Rheum 2005; 52: 3792-3800.

Tunariu N, Gibbs SJR, Win Z, et al. Ventilation-perfusion scintigraphy Is more sensitive than multidetecto CTPA in detecting chronic thromboembolic pulmonary disease as a treatable cause of pulmonary hypertension. $J$ Nucl Med 2007; 48: 680-684.

Meng JJ, Zhang LJ, Wang Q, et al. [A comparison of ventilation/perfusion single photon emission CT and CT pulmonary angiography for diagnosis of pulmonary embolism]. Zhonghua Jie $\mathrm{He} \mathrm{He} \mathrm{Hu} \mathrm{Xi} \mathrm{Za} \mathrm{Zhi} \mathrm{2013;} \mathrm{36:}$ $177-181$.

Rajaram S, Swift AJ, Telfer A, et al. 3D contrast-enhanced lung perfusion MRI is an effective screening tool for chronic thromboembolic pulmonary hypertension: results from the ASPIRE Registry. Thorax 2013; 68: 677-678.

Rajaram S, Swift AJ, Condliffe R, et al. CT features of pulmonary arterial hypertension and its major subtypes: a systematic CT evaluation of 292 patients from the ASPIRE Registry. Thorax 2015; 70: 382-387.

Shen Y, Wan C, Tian P, et al. CT-base pulmonary artery measurement in the detection of pulmonary hypertension: a meta-analysis and systematic review. Medicine (Baltimore) 2014; 93: e256.

Tan RT, Kuzo R, Goodman LR, et al. Utility of CT scan evaluation for predicting pulmonary hypertension in patients with parenchymal lung disease. Chest 1998; 113: 1250-1256.

Resten A, Maitre S, Humbert M, et al. Pulmonary hypertension: CT of the chest in pulmonary venoocclusive disease. Am J Roentgenol 2004; 183: 65-70.

Dartevelle P, Fadel E, Mussot S, et al. Chronic thromboembolic pulmonary hypertension. Eur Respir J 2004; 23 : 637-648.

Reichelt A, Hoeper MM, Galanski M, et al. Chronic thromboembolic pulmonary hypertension: evaluation with 64-detector row CT versus digital substraction angiography. Eur J Radiol 2008; 71: 49-54.

Fedullo PF, Auger WR, Kerr KM, et al. Chronic thromboembolic pulmonary hypertension. N Engl J Med 2001; 345: 1465-1472.

Fukui S, Ogo T, Morita Y, et al. Right ventricular reverse remodelling after balloon pulmonary angioplasty. Eur Respir J 2014; 43: 1394-1402.

Castaner E, Alguersuari A, Andreu M, et al. Imaging findings in pulmonary vasculitis. Semin Ultrasound CT MR 2012; 33: 567-579.

Nawaz A, Litt HI, Stavropoulos SW, et al. Digital subtraction pulmonary arteriography versus multidetector CT in the detection of pulmonary arteriovenous malformations. J Vasc Interv Radiol 2008; 19: 1582-1588.

Peacock AJ, Vonk Noordegraaf A. Cardiac magnetic resonance imaging in pulmonary arterial hypertension. Eur Respir Rev 2013; 22: 526-534.

Swift AJ, Rajaram S, Condliffe R, et al. Diagnostic accuracy of cardiovascular magnetic resonance imaging of right ventricular morphology and function in the assessment of suspected pulmonary hypertension results from the ASPIRE registry. J Cardiovasc Magn Reson 2012; 14: 40-50.

Swift AJ, Rajaram S, Hurdman J, et al. Noninvasive estimation of PA pressure, flow, and resistance with CMR imaging: derivation and prospective validation study from the ASPIRE registry. JACC Cardiovasc Imaging 2013; 6: $1036-1047$

Ley S, Kauczor HU, Heussel CP, et al. Value of contrast-enhanced MR angiography and helical CT angiography in chronic thromboembolic pulmonary hypertension. Eur Radiol 2003; 13: 2365-2371.

van Wolferen SA, Marcus JT, Boonstra A, et al. Prognostic value of right ventricular mass, volume, and function in idiopathic pulmonary arterial hypertension. Eur Heart J 2007; 28: 1250-1257.

Peacock AJ, Crawley S, McLure L, et al. Changes in right ventricular function measured by cardiac magnetic resonance imaging in patients receiving pulmonary arterial hypertension-targeted therapy: the EURO-MR Study. Circ Cardiovasc Imaging 2014; 7: 107-114.

van de Veerdonk MC, Kind T, Marcus JT, et al. Progressive right ventricular dysfunction in patients with pulmonary arterial hypertension responding to therapy. J Am Coll Cardiol 2011; 58: 2511-2519.

Albrecht T, Blomley MJ, Cosgrove DO, et al. Non-invasive diagnosis of hepatic cirrhosis by transit-time analysis of an ultrasound contrast agent. Lancet 1999; 353: 1579-1583.

Naeije R. Hepatopulmonary syndrome and portopulmonary hypertension. Swiss Med Wkly 2003; 133: 163-169. 
Hoeper MM, Lee SH, Voswinckel R, et al. Complications of right heart catheterization procedures in patients with pulmonary hypertension in experienced centers. J Am Coll Cardiol 2006; 48: 2546-2552.

Kovacs G, Avian A, Pienn M, et al. Reading pulmonary vascular pressure tracings. How to handle the problems of zero leveling and respiratory swings. Am J Respir Crit Care Med 2014; 190: 252-257.

Hoeper MM, Maier R, Tongers J, et al. Determination of cardiac output by the Fick method, thermodilution, and acetylene rebreathing in pulmonary hypertension. Am J Respir Crit Care Med 1999; 160: 535-541.

Frost AE, Farber HW, Barst RJ, et al. Demographics and outcomes of patients diagnosed with pulmonary hypertension with pulmonary capillary wedge pressures 16 to $18 \mathrm{~mm} \mathrm{Hg}$ : insights from the REVEAL Registry. Chest 2013; 143: 185-195.

Abraham WT, Adamson PB, Bourge RC, et al. Wireless pulmonary artery haemodynamic monitoring in chronic heart failure: a randomised controlled trial. Lancet 2011; 377: 658-666.

Prasad A, Hastings JL, Shibata S, et al. Characterization of static and dynamic left ventricular diastolic function in patients with heart failure with a preserved ejection fraction. Circ Heart Fail 2010; 3: 617-626.

Fujimoto N, Borlaug BA, Lewis GD, et al. Hemodynamic responses to rapid saline loading: the impact of age, sex, and heart failure. Circulation 2013; 127: 55-62.

Fox BD, Shimony A, Langleben D, et al. High prevalence of occult left heart disease in scleroderma-pulmonary hypertension. Eur Respir J 2013; 42: 1083-1091.

Robbins IM, Hemnes AR, Pugh ME, et al. High prevalence of occult pulmonary venous hypertension revealed by fluid challenge in pulmonary hypertension. Circ Heart Fail 2014; 7: 116-122.

Borlaug BA, Nishimura RA, Sorajja P, et al. Exercise hemodynamics enhance diagnosis of early heart failure with preserved ejection fraction. Circ Heart Fail 2010; 3: 588-595.

Hager WD, Collins I, Tate JP, et al. Exercise during cardiac catheterization distinguishes between pulmonary and left ventricular causes of dyspnea in systemic sclerosis patients. Clin Respir J 2013; 7: 227-236.

Halpern SD, Taichman DB. Misclassification of pulmonary hypertension due to reliance on pulmonary capillary wedge pressure rather than left ventricular end-diastolic pressure. Chest 2009; 136: 37-43.

Naeije R, Vachiery JL, Yerly P, et al. The transpulmonary pressure gradient for the diagnosis of pulmonary vascular disease. Eur Respir J 2013; 41: 217-223.

Provencher S, Herve P, Sitbon O, et al. Changes in exercise haemodynamics during treatment in pulmonary arterial hypertension. Eur Respir J 2008; 32: 393-398.

Tedford RJ, Beaty CA, Mathai SC, et al. Prognostic value of the pre-transplant diastolic pulmonary artery pressure-to-pulmonary capillary wedge pressure gradient in cardiac transplant recipients with pulmonary hypertension. J Heart Lung Transplant 2014; 33: 289-297.

Rich S, Kaufmann E, Levy PS. The effect of high doses of calcium-channel blockers on survival in primary pulmonary hypertension [see comments]. N Engl J Med 1992; 327: 76-81.

Sitbon $\mathrm{O}$, Humbert $\mathrm{M}$, Jaïs $\mathrm{X}$, et al. Long-term response to calcium channel blockers in idiopathic pulmonary arterial hypertension. Circulation 2005; 111: 3105-3111.

Barst R, McGoon M, Torbicki A, et al. Diagnosis and differential assessment of pulmonary arterial hypertension. J Am Coll Cardiol 2004;43(Suppl 1):S40-S47.

Morgan JM, McCormack DG, Griffiths MJ, et al. Adenosine as a vasodilator in primary pulmonary hypertension [see comments]. Circulation 1991; 84: 1145-1149.

Nootens M, Schrader B, Kaufmann E, et al. Comparative acute effects of adenosine and prostacyclin in primary pulmonary hypertension. Chest 1995; 107: 54-57.

Hoeper MM, Olschewski H, Ghofrani HA, et al. A comparison of the acute hemodynamic effects of inhaled nitric oxide and aerosolized iloprost in primary pulmonary hypertension. J Am Coll Cardiol 2000; 35: 176-182. Opitz CF, Wensel R, Bettmann M, et al. Assessment of the vasodilator response in primary pulmonary hypertension. Comparing prostacyclin and iloprost administered by either infusion or inhalation. Eur Heart J 2003; 24: 356-365.

Kim NH, Delcroix M, Jenkins DP, et al. Chronic thromboembolic pulmonary hypertension. J Am Coll Cardiol 2013; 62: D92-D99.

Montani D, Price LC, Dorfmuller P, et al. Pulmonary veno-occlusive disease. Eur Respir J 2009; 33: 189-200.

He J, Fang W, Lv B, et al. Diagnosis of chronic thromboembolic pulmonary hypertension: comparison of ventilation/perfusion scanning and multidetector computed tomography pulmonary angiography with pulmonary angiography. Nucl Med Commun 2012; 33: 459-463.

Cottin V, Le Pavec J, Prevot G, et al. Pulmonary hypertension in patients with combined pulmonary fibrosis and emphysema syndrome. Eur Respir J 2010; 35: 105-111.

Taichman DB, McGoon MD, Harhay MO, et al. Wide variation in clinicians' assessment of New York Heart Association/World Health Organization functional class in patients with pulmonary arterial hypertension. Mayo Clin Proc 2009; 84: 586-592.

Sitbon $\mathrm{O}$, Humbert $\mathrm{M}$, Nunes $\mathrm{H}$, et al. Long-term intravenous epoprostenol infusion in primary pulmonary hypertension: prognostic factors and survival. J Am Coll Cardiol 2002; 40: 780-788.

Nickel N, Golpon H, Greer M, et al. The prognostic impact of follow-up assessments in patients with idiopathic pulmonary arterial hypertension. Eur Respir J 2012; 39: 589-596.

Barst RJ, Chung L, Zamanian RT, et al. Functional class improvement and 3-year survival outcomes in patients with pulmonary arterial hypertension in the REVEAL Registry. Chest 2013; 144: 160-168.

Benza RL, Miller DP, Gomberg-Maitland M, et al. Predicting survival in pulmonary arterial hypertension: insights from the Registry to Evaluate Early and Long-Term Pulmonary Arterial Hypertension Disease Management (REVEAL). Circulation 2010; 122: 164-172.

00 McLaughlin VV, Sitbon O, Badesch DB, et al. Survival with first-line bosentan in patients with primary pulmonary hypertension. Eur Respir J 2005; 25: 244-249.

Sachdev A, Villarraga HR, Frantz RP, et al. Right ventricular strain for prediction of survival in patients with pulmonary arterial hypertension. Chest 2011; 139: 1299-1309.

Raymond RJ, Hinderliter AL, Willis PW, et al. Echocardiographic predictors of adverse outcomes in primary pulmonary hypertension. J Am Coll Cardiol 2002; 39: 1214-1219.

Bustamante-Labarta M, Perrone S, De La Fuente RL, et al. Right atrial size and tricuspid regurgitation severity predict mortality or transplantation in primary pulmonary hypertension. J Am Soc Echocardiogr 2002; 15: 1160-1164. 
104 Forfia PR, Fisher MR, Mathai SC, et al. Tricuspid annular displacement predicts survival in pulmonary hypertension. Am J Respir Crit Care Med 2006; 174: 1034-1041

105 Thenappan T, Shah SJ, Rich S, et al. Survival in pulmonary arterial hypertension: a reappraisal of the NIH risk stratification equation. Eur Respir J 2010; 35: 1079-1087.

106 Wensel R, Opitz CF, Anker SD, et al. Assessment of survival in patients with primary pulmonary hypertension: importance of cardiopulmonary exercise testing. Circulation 2002; 106: 319-324.

107 McLaughlin VV, Shillington A, Rich S. Survival in primary pulmonary hypertension: the impact of epoprostenol therapy. Circulation 2002; 106: 1477-1482.

108 Fine NM, Chen L, Bastiansen PM, et al. Outcome prediction by quantitative right ventricular function assessment in 575 subjects evaluated for pulmonary hypertension. Circ Cardiovasc Imaging 2013; 6: 711-721.

109 Shimada YJ, Shiota M, Siegel RJ, et al. Accuracy of right ventricular volumes and function determined by three-dimensional echocardiography in comparison with magnetic resonance imaging: a meta-analysis study. J Am Soc Echocardiogr 2010; 23: 943-953.

110 Smith BC, Dobson G, Dawson D, et al. Three-dimensional speckle tracking of the right ventricle: toward optimal quantification of right ventricular dysfunction in pulmonary hypertension. J Am Coll Cardiol 2014; 64: 41-51.

111 Grunig E, Tiede H, Enyimayew EO, et al. Assessment and prognostic relevance of right ventricular contractile reserve in patients with severe pulmonary hypertension. Circulation 2013; 128: 2005-2015.

112 Swift AJ, Rajaram S, Marshall H, et al. Black blood MRI has diagnostic and prognostic value in the assessment of patients with pulmonary hypertension. Eur Radiol 2012; 22: 695-702.

113 Swift AJ, Rajaram S, Campbell MJ, et al. Prognostic value of cardiovascular magnetic resonance imaging measurements corrected for age and sex in idiopathic pulmonary arterial hypertension. Circ Cardiovasc Imaging 2014; 7: 100-106.

114 Sitbon O, McLaughlin VV, Badesch DB, et al. Survival in patients with class III idiopathic pulmonary arterial hypertension treated with first line oral bosentan compared with an historical cohort of patients started on intravenous epoprostenol. Thorax 2005; 60: 1025-1030.

115 Rich JD, Archer SL, Rich S. Noninvasive cardiac output measurements in patients with pulmonary hypertension. Eur Respir J 2013; 42: 125-133.

116 Savarese G, Paolillo S, Costanzo P, et al. Do changes of 6-minute walk distance predict clinical events in patients with pulmonary arterial hypertension?: a meta-analysis of 22 randomized trials. J Am Coll Cardiol 2012; 60: 1192-1201.

117 Gabler NB, French B, Strom BL, et al. Validation of 6-minute walk distance as a surrogate end point in pulmonary arterial hypertension trials. Circulation 2012; 126: 349-356.

118 Fritz JS, Blair C, Oudiz RJ, et al. Baseline and follow-up 6-min walk distance and brain natriuretic peptide predict 2-year mortality in pulmonary arterial hypertension. Chest 2013; 143: 315-323.

119 Paciocco G, Martinez F, Bossone E, et al. Oxygen desaturation on the six-minute walk test and mortality in untreated primary pulmonary hypertension. Eur Respir J 2001; 17: 647-652.

120 Provencher $\mathrm{S}$, Chemla $\mathrm{D}$, Herve $\mathrm{P}$, et al. Heart rate responses during the 6-minute walk test in pulmonary arterial hypertension. Eur Respir J 2006; 27: 114-120.

121 Sun XG, Hansen JE, Oudiz RJ, et al. Exercise pathophysiology in patients with primary pulmonary hypertension. Circulation 2001; 104: 429-435.

122 Wensel R, Francis DP, Meyer FJ, et al. Incremental prognostic value of cardiopulmonary exercise testing and resting haemodynamics in pulmonary arterial hypertension. Int J Cardiol 2013; 167: 1193-1198.

123 Blumberg FC, Arzt M, Lange $\mathrm{T}$, et al. Impact of right ventricular reserve on exercise capacity and survival in patients with pulmonary hypertension. Eur J Heart Fail 2013; 15: 771-775.

124 Diller GP, Dimopoulos K, Okonko D, et al. Exercise intolerance in adult congenital heart disease: comparative severity, correlates, and prognostic implication. Circulation 2005; 112: 828-835.

125 Arena R, Lavie CJ, Milani RV, et al. Cardiopulmonary exercise testing in patients with pulmonary arterial hypertension: an evidence-based review. J Heart Lung Transplant 2010; 29: 159-173.

126 Pullamsetti S, Kiss L, Ghofrani HA, et al. Increased levels and reduced catabolism of asymmetric and symmetric dimethylarginines in pulmonary hypertension. FASEB J 2005; 19: 1175-1177.

127 Kielstein JT, Bode-Boger SM, Hesse G, et al. Asymmetrical dimethylarginine in idiopathic pulmonary arterial hypertension. Arterioscler Thromb Vasc Biol 2005; 25: 1414-1418.

128 Kielstein JT, Impraim B, Simmel S, et al. Cardiovascular effects of systemic nitric oxide synthase inhibition with asymmetrical dimethylarginine in humans. Circulation 2004; 109: 172-177.

129 Kawut SM, Horn EM, Berekashvili KK, et al. von Willebrand factor independently predicts long-term survival in patients with pulmonary arterial hypertension. Chest 2005; 128: 2355-2362.

130 Kumpers $\mathrm{P}$, Nickel N, Lukasz A, et al. Circulating angiopoietins in idiopathic pulmonary arterial hypertension. Eur Heart J 2010; 31: 2291-2300.

131 Rubens C, Ewert R, Halank M, et al. Big endothelin-1 and endothelin-1 plasma levels are correlated with the severity of primary pulmonary hypertension. Chest 2001; 120: 1562-1569.

132 Quarck R, Nawrot T, Meyns B, et al. C-reactive protein: a new predictor of adverse outcome in pulmonary arterial hypertension. J Am Coll Cardiol 2009; 53: 1211-1218.

133 Balabanian K, Foussat A, Dorfmuller P, et al. CX(3)C chemokine fractalkine in pulmonary arterial hypertension. Am J Respir Crit Care Med 2002; 165: 1419-1425.

134 Dorfmuller P, Zarka V, Durand-Gasselin I, et al. Chemokine RANTES in severe pulmonary arterial hypertension. Am J Respir Crit Care Med 2002; 165: 534-539.

135 Humbert M, Monti G, Brenot F, et al. Increased interleukin-1 and interleukin-6 serum concentrations in severe primary pulmonary hypertension. Am J Respir Crit Care Med 1995; 151: 1628-1631.

136 Nagaya N, Nishikimi T, Uematsu M, et al. Plasma brain natriuretic peptide as a prognostic indicator in patients with primary pulmonary hypertension. Circulation 2000; 102: 865-870.

137 Leuchte HH, El Nounou M, Tuerpe JC, et al. N-terminal pro-brain natriuretic peptide and renal insufficiency as predictors of mortality in pulmonary hypertension. Chest 2007; 131: 402-409.

138 Fijalkowska A, Kurzyna M, Torbicki A, et al. Serum N-terminal brain natriuretic peptide as a prognostic parameter in patients with pulmonary hypertension. Chest 2006; 129: 1313-1321.

139 Torbicki A, Kurzyna M, Kuca P, et al. Detectable serum cardiac troponin $\mathrm{T}$ as a marker of poor prognosis among patients with chronic precapillary pulmonary hypertension. Circulation 2003; 108: 844-848. 

hypertension. Am I Respir Crit Care Med 2008; 178: 534-541.

Nagaya N, Uematsu M, Satoh T, et al. Serum uric acid levels correlate with the severity and the mortality of primary pulmonary hypertension. Am J Respir Crit Care Med 1999; 160: 487-492.

Lorenzen JM, Nickel N, Kramer R, et al. Osteopontin in patients with idiopathic pulmonary hypertension. Chest 2011; 139: 1010-1017.

Warwick G, Thomas PS, Yates DH. Biomarkers in pulmonary hypertension. Eur Respir J 2008; 32: 503-512. pulmonary arterial hypertension. Eur Respir J 2005; 26: 858-863.

McLaughlin VV, Gaine SP, Howard LS, et al. Treatment goals of pulmonary hypertension. J Am Coll Cardiol 2013;62(Suppl):D73-D81.

Galiè N, Manes A, Negro L, et al. A meta-analysis of randomized controlled trials in pulmonary arterial hypertension. Eur Heart J 2009; 30: 394-403.

Bai Y, Sun L, Hu S, et al. Combination therapy in pulmonary arterial hypertension: a meta-analysis. Cardiology 2011; 120: 157-165

Galiè N, Simonneau G. The Fifth World Symposium on Pulmonary Arterial Hypertension. J Am Coll Cardiol 2013;62(Suppl):D1-D3.

Galiè N, Corris P, Frost A, et al. Updated treatment algorithm of pulmonary hypertension. J Am Coll Cardiol 2013;62(Suppl):D60-D72.

Löwe B, Gräfe K, Ufer C, et al. Anxiety and depression in patients with pulmonary hypertension. Psychosom Med 2004; 66: 831-836.

Galiè N, Hoeper M, Humbert M, et al. Guidelines on diagnosis and treatment of pulmonary hypertension: the Task Force on Diagnosis and Treatment of Pulmonary Hypertension of the European Society of Cardiology and of the European Respiratory Society. Eur Heart J 2009; 30: 2493-2537.

Mereles D, Ehlken N, Kreuscher S, et al. Exercise and respiratory training improve exercise capacity and quality of life in patients with severe chronic pulmonary hypertension. Circulation 2006; 114: 1482-1489.

de Man FS, Handoko ML, Groepenhoff $\mathrm{H}$, et al. Effects of exercise training in patients with idiopathic pulmonary arterial hypertension. Eur Respir J 2009; 34: 669-675.

Grunig E, Ehlken N, Ghofrani A, et al. Effect of exercise and respiratory training on clinical progression and survival in patients with severe chronic pulmonary hypertension. Respiration 2011; 81: 394-401.

Grunig E, Maier F, Ehlken N, et al. Exercise training in pulmonary arterial hypertension associated with connective tissue diseases. Arthritis Res Ther 2012; 14: R148.

Grunig E, Lichtblau M, Ehlken N, et al. Safety and efficacy of exercise training in various forms of pulmonary hypertension. Eur Respir J 2012; 40: 84-92.

Becker-Grunig T, Klose $\mathrm{H}$, Ehlken $\mathrm{N}$, et al. Efficacy of exercise training in pulmonary arterial hypertension associated with congenital heart disease. Int J Cardiol 2013; 168: 375-381.

Weinstein AA, Chin LMK, Keyser RE, et al. Effect of aerobic exercise training on fatigue and physical activity in patients with pulmonary arterial hypertension. Respir Med 2013; 107: 778-784.

Chan L, Chin LM, Kennedy M, et al. Benefits of intensive treadmill exercise training on cardiorespiratory function and quality of life in patients with pulmonary hypertension. Chest 2013; 143: 333-343.

Jaï X, Olsson KM, Barbera JA, et al. Pregnancy outcomes in pulmonary arterial hypertension in the modern management era. Eur Respir J 2012; 40: 881-885.

Duarte AG, Thomas S, Safdar Z, et al. Management of pulmonary arterial hypertension during pregnancy: a retrospective, multicenter experience. Chest 2013; 143: 1330-1336.

Thorne S, Nelson-Piercy C, MacGregor AJ, et al. Pregnancy and contraception in heart disease and pulmonary arterial hypertension. J Fam Plann Reprod Health Care 2006; 32: 75-81.

Bendayan D, Hod M, Oron G, et al. Pregnancy outcome in patients with pulmonary arterial hypertension receiving prostacyclin therapy. Obstet Gynecol 2005; 106: 1206-1210.

Bonnin M, Mercier FJ, Sitbon O, et al. Severe pulmonary hypertension during pregnancy: mode of delivery and anesthetic management of 15 consecutive cases. Anesthesiology 2005; 102: 1133-1137.

Meyer S, McLaughlin VV, Seyfarth HJ, et al. Outcomes of noncardiac, nonobstetric surgery in patients with PAH: an international prospective survey. Eur Respir J 2013; 41: 1302-1307.

Olofsson C, Bremme K, Forssell G, et al. Cesarean section under epidural ropivacaine $0.75 \%$ in a parturient with severe pulmonary hypertension. Acta Anaesthesiol Scand 2001; 45: 258-260.

Raines DE, Liberthson RR, Murray JR. Anesthetic management and outcome following noncardiac surgery in nonparturients with Eisenmenger's physiology. J Clin Anesth 1996; 8: 341-347.

Guillevin L, Armstrong I, Aldrighetti R, et al. Understanding the impact of pulmonary arterial hypertension on patients' and carers' lives. Eur Respir Rev 2013; 22: 535-542.

Weitzenblum E, Sautegeau A, Ehrhart M, et al. Long-term oxygen therapy can reverse the progression of pulmonary hypertension in patients with chronic obstructive pulmonary disease. Am Rev Respir Dis 1985; 131: 493-498.

70 Frydman N, Steffann J, Girerd B, et al. Pre-implantation genetic diagnosis in pulmonary arterial hypertension due to BMPR2 mutation. Eur Respir J 2012; 39: 1534-1535.

Fuster V, Steele PM, Edwards WD, et al. Primary pulmonary hypertension: natural history and the importance of thrombosis. Circulation 1984; 70: 580-587.

2 Herve $\mathrm{P}$, Humbert $\mathrm{M}$, Sitbon $\mathrm{O}$, et al. Pathobiology of pulmonary hypertension: the role of platelets and thrombosis. Clin Chest Med 2001; 22: 451-458.

Hoeper MM, Sosada M, Fabel H. Plasma coagulation profiles in patients with severe primary pulmonary hypertension. Eur Respir J 1998; 12: 1446-1449.

Huber K, Beckmann R, Frank H, et al. Fibrinogen, t-PA, and PAI-1 plasma levels in patients with pulmonary hypertension. Am J Respir Crit Care Med 1994; 150: 929-933.

5 Olsson KM, Delcroix M, Ghofrani HA, et al. Anticoagulation and survival in pulmonary arterial hypertension: results from the Comparative, Prospective Registry of Newly Initiated Therapies for Pulmonary Hypertension (COMPERA). Circulation 2014; 129: 57-65. 

with pulmonary arterial hypertension $(\mathrm{PAH})$ : insights from the randomised controlled SERAPHIN trial of macitentan. Eur Heart J 2014; 35: 10.

177 Preston RJ, Roberts KE, Miller DP, et al. Effect of warfarin treatment on survival of patients with pulmonary arterial hypertension (PAH) in the Registry to Evaluate Early and Long-Term PAH Disease Management (REVEAL). Am J Respir Crit Care Med 2014; 189: A2464.

178 Cohn JN. Optimal diuretic therapy for heart failure. Am J Med 2001; 111: 577

179 Sandoval J, Aguirre JS, Pulido T, et al. Nocturnal oxygen therapy in patients with the Eisenmenger syndrome. Am J Respir Crit Care Med 2001; 164: 1682-1687.

180 Rich S, Seidlitz M, Dodin E, et al. The short-term effects of digoxin in patients with right ventricular dysfunction from pulmonary hypertension. Chest 1998; 114: 787-792.

181 Ruiter G, Lankhorst S, Boonstra A, et al. Iron deficiency is common in idiopathic pulmonary arterial hypertension. Eur Respir J 2011; 37: 1386-1391.

182 Ruiter G, Lanser IJ, de Man FS, et al. Iron deficiency in systemic sclerosis patients with and without pulmonary hypertension. Rheumatology (Oxford) 2014; 53: 285-292.

183 Broberg CS, Bax BE, Okonko DO, et al. Blood viscosity and its relationship to iron deficiency, symptoms, and exercise capacity in adults with cyanotic congenital heart disease. J Am Coll Cardiol 2006; 48: 356-365.

184 Rhodes CJ, Howard LS, Busbridge M, et al. Iron deficiency and raised hepcidin in idiopathic pulmonary arterial hypertension clinical prevalence, outcomes, and mechanistic insights. J Am Coll Cardiol 2011; 58: 300-309.

185 Van De Bruaene A, Delcroix M, Pasquet A, et al. Iron deficiency is associated with adverse outcome in Eisenmenger patients. Eur Heart J 2011; 32: 2790-2799.

186 Viethen T, Gerhardt F, Dumitrescu D, et al. Ferric carboxymaltose improves exercise capacity and quality of life in patients with pulmonary arterial hypertension and iron deficiency: a pilot study. Int J Cardiol 2014; 175: 233-239. hypertension. Am J Cardiol 1995; 75: 55A-62A.

188 Mukerjee D, St George D, Coleiro B, et al. Prevalence and outcome in systemic sclerosis associated pulmonary arterial hypertension: application of a registry approach. Ann Rheum Dis 2003; 62: 1088-1093.

189 Montani D, Savale L, Natali D, et al. Long-term response to calcium-channel blockers in non-idiopathic pulmonary arterial hypertension. Eur Heart J 2010; 31: 1898-1907.

190 Giaid A, Yanagisawa M, Langleben D, et al. Expression of endothelin-1 in the lungs of patients with pulmonary hypertension. N Engl J Med 1993; 328: 1732-1739.

191 Stewart DJ, Levy RD, Cernacek P, et al. Increased plasma endothelin-1 in pulmonary hypertension: marker or mediator of disease? Ann Intern Med 1991; 114: 464-469.

192 Galié N, Manes A, Branzi A. The endothelin system in pulmonary arterial hypertension. Cardiovasc Res 2004; 61: 227-237.

193 Galié N, Badesch BD, Oudiz R, et al. Ambrisentan therapy for pulmonary arterial hypertension. J Am Coll Cardiol 2005; 46: 529-535.

194 Galié N, Olschewski H, Oudiz RJ, et al. Ambrisentan for the treatment of pulmonary arterial hypertension. Results of the ambrisentan in pulmonary arterial hypertension, randomized, double-blind, placebo-controlled, multicenter, efficacy (ARIES) study 1 and 2. Circulation 2008; 117: 3010-3019.

195 McGoon M, Frost A, Oudiz R, et al. Ambrisentan therapy in patients with pulmonary arterial hypertension who discontinued bosentan or sitaxsentan due to liver function test abnormalities. Chest 2009; 135: 122-129.

196 Channick RN, Simonneau G, Sitbon O, et al. Effects of the dual endothelin-receptor antagonist bosentan in patients with pulmonary hypertension: a randomised placebo-controlled study. Lancet 2001; 358: 1119-1123.

197 Rubin LJ, Badesch DB, Barst RJ, et al. Bosentan therapy for pulmonary arterial hypertension. N Engl J Med 2002; 346: 896-903.

198 Humbert M, Barst RJ, Robbins IM, et al. Combination of bosentan with epoprostenol in pulmonary arterial hypertension: BREATHE-2. Eur Respir J 2004; 24: 353-359.

199 Galiè N, Rubin LJ, Hoeper M, et al. Treatment of patients with mildly symptomatic pulmonary arterial hypertension with bosentan (EARLY study): a double-blind, randomised controlled trial. Lancet 2008; 371: 2093-2100.

200 Galiè N, Beghetti M, Gatzoulis MA, et al. Bosentan therapy in patients with Eisenmenger syndrome: a multicenter, double-blind, randomized, placebo-controlled study. Circulation 2006; 114: 48-54.

201 Pulido T, Adzerikho I, Channick RN, et al. Macitentan and morbidity and mortality in pulmonary arterial hypertension. N Engl J Med 2013; 369: 809-818.

202 Wharton J, Strange JW, Moller GMO, et al. Antiproliferative effects of phosphodiesterase type 5 inhibition in human pulmonary artery cells. Am J Respir Crit Care Med 2005; 172: 105-113.

203 Tantini B, Manes A, Fiumana E, et al. Antiproliferative effect of sildenafil on human pulmonary artery smooth muscle cells. Basic Res Cardiol 2005; 100: 131-138.

204 Ghofrani HA, Voswinckel R, Reichenberger F, et al. Differences in hemodynamic and oxygenation responses to three different phosphodiesterase-5 inhibitors in patients with pulmonary arterial hypertension: a randomized prospective study. J Am Coll Cardiol 2004; 44: 1488-1496.

205 Galiè N, Ghofrani HA, Torbicki A, et al. the Sildenafil Use in Pulmonary Arterial Hypertension (SUPER) Study Group. Sildenafil citrate therapy for pulmonary arterial hypertension. N Engl J Med 2005; 353: 2148-2157.

206 Sastry BKS, Narasimhan C, Reddy NK, et al. Clinical efficacy of sildenafil in primary pulmonary hypertension: a randomized, placebo-controlled, double-blind, crossover study. J Am Coll Cardiol 2004; 43: 1149-1153.

207 Iversen K, Jensen AS, Jensen TV, et al. Combination therapy with bosentan and sildenafil in Eisenmenger syndrome: a randomized, placebo-controlled, double-blinded trial. Eur Heart J 2010; 31: 1124-1131.

208 Singh T, Rohit M, Grover A, et al. A randomized, placebo-controlled, double-blind, crossover study to evaluate the efficacy of oral sildenafil therapy in severe pulmonary artery hypertension. Am Heart J 2006; 151: 851.e1-851.e5. Simonneau G, Rubin L, Galiè N, et al. Addition of sildenafil to long-term intravenous epoprostenol therapy in patients with pulmonary arterial hypertension. Ann Intern Med 2008; 149: 521-530.

210 Vachiery JL, Huez S, Gillies H, et al. Safety, tolerability and pharmacokinetics of an intravenous bolus of sildenafil in patients with pulmonary arterial hypertension. Br J Clin Pharmacol 2011; 71: 289-292. 
211 Galiè N, Brundage BH, Ghofrani HA, et al. Tadalafil therapy for pulmonary arterial hypertension. Circulation 2009; 119: 2894-2903

212 Jing ZC, Yu ZX, Shen JY, et al. Vardenafil in pulmonary arterial hypertension: a randomized, double-blind, placebo-controlled study. Am J Respir Crit Care Med 2011; 183: 1723-1729.

213 Giaid A, Saleh D. Reduced expression of endothelial nitric oxide synthase in the lungs of patients with pulmonary hypertension. N Engl J Med 1995; 333: 214-221.

214 Ghofrani HA, Galiè N, Grimminger F, et al. Riociguat for the treatment of pulmonary arterial hypertension. $N$ Engl J Med 2013; 369: 330-340.

215 Galiè N, Muller K, Scalise AV, et al. PATENT PLUS: a blinded, randomised and extension study of riociguat plus sildenafil in PAH. Eur Respir J 2015; 45: 1314-1322.

216 Jones DA, Benjamin CW, Linseman DA. Activation of thromboxane and prostacyclin receptors elicits opposing effects on vascular smooth muscle cell growth and mitogen-activated protein kinase signaling cascades. $\mathrm{Mol}$ Pharmacol 1995; 48: 890-896.

217 Galiè N, Manes A, Branzi A. Prostanoids for pulmonary arterial hypertension. Am J Respir Med 2003; 2: 123-137.

218 Galiè N, Humbert M, Vachiery JL, et al. Effects of beraprost sodium, an oral prostacyclin analogue, in patients with pulmonary arterial hypertension: a randomised, double-blind placebo-controlled trial. J Am Coll Cardiol 2002; 39: 1496-1502.

219 Barst RJ, McGoon M, McLaughlin VV, et al. Beraprost therapy for pulmonary arterial hypertension. J Am Coll Cardiol 2003; 41: 2119-2125.

220 Rubin LJ, Mendoza J, Hood M, et al. Treatment of primary pulmonary hypertension with continuous intravenous prostacyclin (epoprostenol). Results of a randomized trial. Ann Intern Med 1990; 112: 485-491.

221 Barst RJ, Rubin LJ, Long WA, et al. A comparison of continuous intravenous epoprostenol (prostacyclin) with conventional therapy for primary pulmonary hypertension [see comments]. N Engl J Med 1996; 334: 296-302.

222 Badesch DB, Tapson VF, McGoon MD, et al. Continuous intravenous epoprostenol for pulmonary hypertension due to the scleroderma spectrum of disease. A randomized, controlled trial [see comments]. Ann Intern Med 2000; 132: 425-434.

223 Rosenzweig EB, Kerstein D, Barst RJ. Long-term prostacyclin for pulmonary hypertension with associated congenital heart defects. Circulation 1999; 99: 1858-1865.

224 Krowka MJ, Frantz RP, McGoon MD, et al. Improvement in pulmonary hemodynamics during intravenous epoprostenol (prostacyclin): a study of 15 patients with moderate to severe portopulmonary hypertension. Hepatology 1999; 30: 641-648.

Nunes $\mathrm{H}$, Humbert $\mathrm{M}$, Sitbon $\mathrm{O}$, et al. Prognostic factors for survival in human immunodeficiency virus-associated pulmonary arterial hypertension. Am J Respir Crit Care Med 2003; 167: 1433-1439.

rol S, Souza R, Jais X, et al. Intravenous epoprostenol in inoperable chronic thromboembolic pulmonary hypertension. J Heart Lung Transplant 2007; 26: 357-362.

227 Doran AK, Ivy DD, Barst RJ, et al. Guidelines for the prevention of central venous catheter-related blood stream infections with prostanoid therapy for pulmonary arterial hypertension. Int J Clin Pract Suppl 2008; 160: 5-9.

228 Sitbon O, Delcroix M, Bergot E, et al. EPITOME-2: an open-label study assessing the transition to a new formulation of intravenous epoprostenol in patients with pulmonary arterial hypertension. Am Heart J 2014; 167: 210-217.

Olschewski H, Simonneau G, Galiè N, et al. Inhaled iloprost in severe pulmonary hypertension. N Engl J Med 2002; 347: 322-329.

230 McLaughlin VV, Oudiz RJ, Frost A, et al. Randomized study of adding inhaled iloprost to existing bosentan in pulmonary arterial hypertension. Am J Respir Crit Care Med 2006; 174: 1257-1263.

231 Hoeper M, Leuchte H, Halank M, et al. Combining inhaled iloprost with bosentan in patients with idiopathic pulmonary arterial hypertension. Eur Respir J 2006; 4: 691-694.

232 Higenbottam T, Butt AY, McMahon A, et al. Long-term intravenous prostaglandin (epoprostenol or iloprost) for treatment of severe pulmonary hypertension. Heart 1998; 80: 151-155.

233 Simonneau G, Barst RJ, Galiè N, et al. Continuous subcutaneous infusion of treprostinil, a prostacyclin analogue, in patients with pulmonary arterial hypertension. A double-blind, randomized, placebo-controlled trial. Am J Respir Crit Care Med 2002; 165: 800-804.

234 Hiremath J, Thanikachalam S, Parikh K, et al. Exercise improvement and plasma biomarker changes with intravenous treprostinil therapy for pulmonary arterial hypertension: a placebo-controlled trial. $J$ Heart Lung Transplant 2010; 29: 137-149.

235 Tapson VF, Gomberg-Maitland M, McLaughlin VV, et al. Safety and efficacy of IV treprostinil for pulmonary arterial hypertension: a prospective, multicenter, open-label, 12-week trial. Chest 2006; 129: 683-688.

236 Sitbon O, Manes A, Jaïs X, et al. Rapid switch from intravenous epoprostenol to intravenous treprostinil in patients with pulmonary arterial hypertension. J Cardiovasc Pharmacol 2007; 49: 1-5.

237 McLaughlin V, Rubin L, Benza RL, et al. Addition of inhaled treprostinil to oral therapy for pulmonary arterial hypertension: a randomized controlled clinical trial. J Am Coll Cardiol 2010; 55: 1915-1922.

238 Tapson VF, Torres F, Kermeen F, et al. Oral treprostinil for the treatment of pulmonary arterial hypertension in patients on background endothelin receptor antagonist and/or phosphodiesterase type 5 inhibitor therapy (the FREEDOM-C study): a randomized controlled trial. Chest 2012; 142: 1383-1390.

239 Tapson VF, Jing ZC, Xu KF, et al. Oral treprostinil for the treatment of pulmonary arterial hypertension in patients on background endothelin receptor antagonist and/or phosphodiesterase type 5 inhibitor therapy (the FREEDOM-C2 study): a randomized controlled trial. Chest 2013; 144: 952-958.

240 Jing ZC, Parikh K, Pulido T, et al. Efficacy and safety of oral treprostinil monotherapy for the treatment of pulmonary arterial hypertension: a randomized, controlled trial. Circulation 2013; 127: 624-633.

241 Simonneau G, Torbicki A, Hoeper MM, et al. Selexipag, an oral, selective prostacyclin receptor agonist for the treatment of pulmonary arterial hypertension. Eur Respir J 2012; 40: 874-880.

242 Chen SL, Zhang FF, Xu J, et al. Pulmonary artery denervation to treat pulmonary arterial hypertension: the single-center, prospective, first-in-man PADN-1 study (first-in-man pulmonary artery denervation for treatment of pulmonary artery hypertension). J Am Coll Cardiol 2013; 62: 1092-1100. 
Galiè N, Manes A. New treatment strategies for pulmonary arterial hypertension: hopes or hypes? J Am Coll Cardiol 2013; 62: 1101-1102.

Galiè N, Palazzini M, Manes A. Pulmonary arterial hypertension: from the kingdom of the near-dead to multiple clinical trial meta-analyses. Eur Heart J 2010; 31: 2080-2086.

Kemp K, Savale L, O'Callaghan DS, et al. Usefulness of first-line combination therapy with epoprostenol and bosentan in pulmonary arterial hypertension: an observational study. J Heart Lung Transplant 2012; 31: $150-158$.

Sitbon O, Jaiis X, Savale L, et al. Upfront triple combination therapy in pulmonary arterial hypertension: a pilot study. Eur Respir J 2014; 43: 1691-1697.

Galiè N, Barbera JA, Frost A, et al. Initial Use of Ambrisentan plus Tadalafil in Pulmonary Arterial Hypertension. New Engl J Med 2015; 379: 834-844.

McLaughlin VV, Channick R, Chin KM, et al. Effect of selexipag on morbidity/mortality in pulmonary arterial hypertension: results of the GRIPHON study. J Am Coll Cardiol 2015;65(Suppl A):A380.

Badesch BD, Feldman J, Keogh A, et al. ARIES-3: ambrisentan therapy in a diverse population of patients with pulmonary hypertension. Cardiovasc Ther 2012; 30: 93-99.

Provencher S, Sitbon O, Humbert M, et al. Long-term outcome with first-line bosentan therapy in idiopathic pulmonary arterial hypertension. Eur Heart J 2006; 27: 589-595.

McLaughlin V, Channick RN, Ghofrani HA, et al. Bosentan added to sildenafil therapy in patients with pulmonary arterial hypertension. Eur Respir J 2015; 46: 405-413.

Dardi F, Manes A, Palazzini M, et al. Combining bosentan and sildenafil in pulmonary arterial hypertension patients failing monotherapy: real-world insights. Eur Respir J 2015; 46: 414-421.

Sandoval J, Gaspar J, Pulido T, et al. Graded balloon dilation atrial septostomy in severe primary pulmonary hypertension. A therapeutic alternative for patients nonresponsive to vasodilator treatment. J Am Coll Cardiol 1998; 32: 297-304.

Kurzyna M, Dabrowski M, Bielecki D, et al. Atrial septostomy in treatment of end-stage right heart failure in Patients with pulmonary hypertension. Chest 2007; 131: 977-983.

Althoff TF, Knebel F, Panda A, et al. Long-term follow-up of a fenestrated Amplatzer atrial septal occluder in pulmonary arterial hypertension. Chest 2008; 133: 283-285.

Keogh A, Benza RL, Corris P, et al. Interventional and surgical modalities of treatment in pulmonary arterial hypertension. J Am Coll Cardiol 2009;54(Suppl):S67-S77.

Sztrymf B, Souza R, Bertoletti I, et al. Prognostic factors of acute heart failure in patients with pulmonary arterial hypertension. Eur Respir J 2010; 35: 1286-1293.

Zamanian RT, Haddad F, Doyle RL, et al. Management strategies for patients with pulmonary hypertension in the intensive care unit. Crit Care Med 2007; 35: 2037-2050.

Price LC, Wort SJ, Finney SJ, et al. Pulmonary vascular and right ventricular dysfunction in adult critical care: current and emerging options for management: a systematic literature review. Crit Care 2010; 14: R169.

Hoeper MM, Granton J. Intensive care unit management of patients with severe pulmonary hypertension and right heart failure. Am J Respir Crit Care Med 2011; 184: 1114-1124. for acute right heart failure in group 1 pulmonary arterial hypertension. ASAIO J 2014; 60: 129-133.

Olsson KM, Simon A, Strueber M, et al. Extracorporeal membrane oxygenation in nonintubated patients as bridge to lung transplantation. Am J Transplant 2010; 10: 2173-2178.

Fuehner T, Kuehn C, Hadem J, et al. Extracorporeal membrane oxygenation in awake patients as bridge to lung transplantation. Am J Respir Crit Care Med 2012; 185: 763-768.

de Perrot M, Granton JT, McRae K, et al. Impact of extracorporeal life support on outcome in patients with idiopathic pulmonary arterial hypertension awaiting lung transplantation. J Heart Lung Transplant 2011; 30: 997-1002

Strueber M, Hoeper MM, Fischer S, et al. Bridge to thoracic organ transplantation in patients with pulmonary arterial hypertension using a pumpless lung assist device. Am J Transplant 2009; 9: 853-857.

Trulock EP, Edwards LB, Taylor DO, et al. Registry of the International Society for Heart and Lung Transplantation: twenty-third official adult lung and heart lung transplantation report-2006. J Heart Lung Transplant 2006; 25: 880-892.

Toyoda Y, Thacker J, Santos R, et al. Long-term outcome of lung and heart-lung transplantation for idiopathic pulmonary arterial hypertension. Ann Thorac Surg 2008; 86: 1116-1122.

Fadel E, Mercier O, Mussot S, et al. Long-term outcome of double-lung and heart-lung transplantation for pulmonary hypertension: a comparative retrospective study of 219 patients. Eur J Cardiothorac Surg 2010; 38: 277-284.

de Perrot M, Granton JT, McRae K, et al. Outcome of patients with pulmonary arterial hypertension referred for lung transplantation: a 14-year single-center experience. J Thorac Cardiovasc Surg 2012; 143: 910-918.

Christie JD, Edwards LB, Kucheryavaya AY, et al. The Registry of the International Society for Heart and Lung Transplantation: 29th adult lung and heart-lung transplant report-2012. J Heart Lung Transplant 2012;31: 1073-1086.

1 Waddell TK, Bennett L, Kennedy R, et al. Heart-lung or lung transplantation for Eisenmenger syndrome. J Heart Lung Transplant 2002; 21: 731-737.

Choong CK, Sweet SC, Guthrie TJ, et al. Repair of congenital heart lesions combined with lung transplantation for the treatment of severe pulmonary hypertension: a 13-year experience. J Thorac Cardiovasc Surg 2005; 129: 661-669.

Fleming TR, Powers JH. Biomarkers and surrogate endpoints in clinical trials. Statistic Med 2012; 31: 2973-2984. Fleming TR. Surrogate endpoints and FDA accelerated approval process. Health Affairs 2005; 24: 67-78.

Tongers J, Schwerdtfeger B, Klein G, et al. Incidence and clinical relevance of supraventricular tachyarrhythmias in pulmonary hypertension. Am Heart J 2007; 153: 127-132.

Hoeper MM, Galiè N, Murali S, et al. Outcome after cardiopulmonary resuscitation in patients with pulmonary arterial hypertension. Am J Respir Crit Care Med 2002; 165: 341-344. 

clinical improvement in patients with persistent atrial flutter and severe pulmonary arterial hypertension. Int $J$ Cardiol 2011; 149: 279-280.

278 Zylkowska J, Kurzyna M, Pietura R, et al. Recurrent hemoptysis: an emerging life-threatening complication in idiopathic pulmonary arterial hypertension. Chest 2011; 139: 690-693.

279 Zylkowska J, Kurzyna M, Florczyk M, et al. Pulmonary artery dilatation correlates with the risk of unexpected death in chronic arterial or thromboembolic pulmonary hypertension. Chest 2012; 142: 1406-1416.

280 Russo V, Zompatori M, Galiè N. Extensive right pulmonary artery dissection in a young patient with chronic pulmonary hypertension. Heart 2012; 98: 265-266.

281 Demerouti EA, Manginas AN, Athanassopoulos GD, et al. Complications leading to sudden cardiac death in pulmonary arterial hypertension. Respiratory Care 2013; 58: 1246-1254.

282 Lee MS, Oyama J, Bhatia R, et al. Left main coronary artery compression from pulmonary artery enlargement due to pulmonary hypertension: a contemporary review and argument for percutaneous revascularization. Catheter Cardiovasc Interv 2010; 76: 543-550.

283 Barst RJ, Ertel SI, Beghetti M, et al. Pulmonary arterial hypertension: a comparison between children and adults. Eur Respir J 2011; 37: 665-677.

284 Barst RJ, McGoon MD, Elliott CG, et al. Survival in childhood pulmonary arterial hypertension. Circulation 2012; 125: 113-122.

285 van Loon RL, Roofthooft MTR, Hillege HL, et al. Pediatric pulmonary hypertension in the Netherlands: epidemiology and characterization during the period 1991 to 2005. Circulation 2011; 124: 1755-1764. Moledina S, Hislop AA, Foster H, et al. Childhood idiopathic pulmonary arterial hypertension: a national cohort study. Heart 2010; 96: 1401-1406.

287 Berger RM, Beghetti M, Humpl T, et al. Clinical features of paediatric pulmonary hypertension: a registry study. Lancet 2012; 379: 537-546.

288 Hansmann G, Hoeper MM. Registries for paediatric pulmonary hypertension. Eur Respir J 2013; 42: $580-583$.

289 Cerro MJ, Abman S, Diaz G, et al. A consensus approach to the classification of pediatric pulmonary hypertensive vascular disease: report from the PVRI Pediatric Taskforce, Panama 2011. Pulm Circ 2011; 1: 286-298.

290 Beghetti M, Berger RM, Schulze-Neick I, et al. Diagnostic evaluation of paediatric pulmonary hypertension in current clinical practice. Eur Respir J 2013; 42: 689-700.

291 Schulze-Neick I, Beghetti M. Issues related to the management and therapy of paediatric pulmonary hypertension. Eur Respir Rev 2010; 19: 331-339.

292 Adatia I, Haworth SG, Wegner M, et al. Clinical trials in neonates and children: report of the Pulmonary Hypertension Academic Research Consortium Pediatric Advisory Committee. Pulm Circ 2013; 3: 252-266.

293 Lammers AE, Hislop AA, Flynn Y, et al. Epoprostenol treatment in children with severe pulmonary hypertension. Heart 2007; 93: 739-743.

294 Barst RJ, Maislin G, Fishman AP. Vasodilator therapy for primary pulmonary hypertension in children. Circulation 1999; 99: 1197-1208.

295 Levy M, Celermajer DS, Bourges-Petit E, et al. Add-on therapy with subcutaneous treprostinil for refractory pediatric pulmonary hypertension. J Pediatr 2011; 158: 584-588.

296 Ivy DD, Doran AK, Smith KJ, et al. Short- and long-term effects of inhaled iloprost therapy in children with pulmonary arterial hypertension. J Am Coll Cardiol 2008; 51: 161-169.

297 Barst R, Ivy D, Widlitz AC, et al. Pharmacokinetics, safety, and efficacy of bosentan in pediatric patients with pulmonary arterial hypertension. Clin Pharmacol Ther 2003; 73: 372-382.

298 Rosenzweig EB, Ivy DD, Widlitz A, et al. Effects of long-term bosentan in children with pulmonary arterial hypertension. J Am Coll Cardiol 2005; 46: 697-704.

299 Beghetti M, Haworth SG, Bonnet D, et al. Pharmacokinetic and clinical profile of a novel formulation of bosentan in children with pulmonary arterial hypertension: the FUTURE-1 study. Br J Clin Pharmacol 2009; 68: 948-955.

300 Barst RJ, Ivy DD, Gaitan G, et al. A randomized, double-blind, placebo-controlled, dose-ranging study of oral sildenafil citrate in treatment-naive children with pulmonary arterial hypertension. Circulation 2012; 125: 324-334.

301 Barst RJ, Beghetti M, Pulido T, et al. STARTS-2: long-term survival with oral sildenafil monotherapy in treatment-naive pediatric pulmonary arterial hypertension. Circulation 2014; 129: 1914-1923.

302 Takatsuki S, Calderbank M, Ivy DD. Initial experience with tadalafil in pediatric pulmonary arterial hypertension. Pediatr Cardiol 2012; 33: 683-688.

303 Douwes JM, Roofthooft MT, Van Loon RL, et al. Sildenafil add-on therapy in paediatric pulmonary arterial hypertension, experiences of a national referral centre. Heart 2014; 100: 224-230.

304 Micheletti A, Hislop AA, Lammers A, et al. Role of atrial septostomy in the treatment of children with pulmonary arterial hypertension. Heart 2006; 92: 969-972.

305 Boudjemline Y, Patel M, Malekzadeh-Milani S, et al. Patent ductus arteriosus stenting (transcatheter Potts shunt) for palliation of suprasystemic pulmonary arterial hypertension: a case series. Circ Cardiovasc Interv 2013; 6: e18-e20.

306 Baruteau AE, Belli E, Boudjemline $\mathrm{Y}$, et al. Palliative Potts shunt for the treatment of children with drug-refractory pulmonary arterial hypertension: updated data from the first 24 patients. Eur J Cardiothorac Surg 2015; 47: e105-e110.

307 Esch JJ, Shah PB, Cockrill BA, et al. Transcatheter Potts shunt creation in patients with severe pulmonary arterial hypertension: initial clinical experience. J Heart Lung Transplant 2013; 32: 381-387.

308 Ploegstra MJ, Douwes JM, Roofthooft MT, et al. Identification of treatment goals in paediatric pulmonary arterial hypertension. Eur Respir J 2014; 44: 1616-1626.

309 Galiè N, Manes A, Palazzini M, et al. Management of pulmonary arterial hypertension associated with congenital systemic-to-pulmonary shunts and Eisenmenger's syndrome. Drugs 2008; 68: 1049-1066.

310 Engelfriet PM, Duffels MGJ, Moller T, et al. Pulmonary arterial hypertension in adults born with a heart septal defect: the Euro Heart Survey on adult congenital heart disease. Heart 2007; 93: 682-687.

311 Beghetti M, Galiè N. Eisenmenger syndrome: a clinical perspective in a new therapeutic era of pulmonary arterial hypertension. J Am Coll Cardiol 2009; 53: 733-740. 
312 Daliento L, Somerville J, Presbitero P, et al. Eisenmenger syndrome. Factors relating to deterioration and death. Eur Heart J 1998; 19: 1845-1855.

313 Hopkins WE, Ochoa LL, Richardson GW, et al. Comparison of the hemodynamics and survival of adults with severe primary pulmonary hypertension or Eisenmenger syndrome. J Heart Lung Transplant 1996; 15: 100-105.

314 Manes A, Palazzini M, Leci E, et al. Current era survival of patients with pulmonary arterial hypertension associated with congenital heart disease: a comparison between clinical subgroups. Eur Heart J 2014; 35: 716-724.

315 Hopkins WE. The remarkable right ventricle of patients with Eisenmenger syndrome. Coron Artery Dis 2005; 16: $19-25$.

316 Diller GP, Gatzoulis MA. Pulmonary vascular disease in adults with congenital heart disease. Circulation 2007; 115: 1039-1050.

317 Moller JH, Patton C, Varco RL, et al. Late results (30 to 35 years) after operative closure of isolated ventricular septal defect from 1954 to 1960. Am J Cardiol 1991; 68: 1491-1497.

318 van Albada ME, Berger RM. Pulmonary arterial hypertension in congenital cardiac disease-the need for refinement of the Evian-Venice classification. Cardiol Young 2008; 18: 10-17.

319 Schulze-Neick I, Beghetti M. Classifying pulmonary hypertension in the setting of the congenitally malformed heart-cleaning up a dog's dinner. Cardiol Young 2008; 18: 22-25.

320 Lopes AA, Leary PW. Measurement, interpretation and use of hemodynamic parameters. Cardiol Young 2009;19 (Suppl 1):8-12.

321 Broberg CS, Ujita M, Prasad S, et al. Pulmonary arterial thrombosis in Eisenmenger syndrome is associated with biventricular dysfunction and decreased pulmonary flow velocity. J Am Coll Cardiol 2007; 50: 634-642.

322 Gatzoulis MA, Beghetti M, Galiè N, et al. Longer-term bosentan therapy improves functional capacity in Eisenmenger syndrome: results of the BREATHE-5 open-label extension study. Int J Cardiol 2007; 127: 27-32.

323 Zuckerman WA, Leaderer D, Rowan CA, et al. Ambrisentan for pulmonary arterial hypertension due to congenital heart disease. J Am Coll Cardiol 2011; 107: 1381-1385.

324 Mukhopadhyay S, Sharma M, Ramakrishnan S, et al. Phosphodiesterase-5 inhibitor in Eisenmenger syndrome: a preliminary observational study. Circulation 2006; 114: 1807-1810.

325 Khanna D, Gladue H, Channick R, et al. Recommendations for screening and detection of connective tissue disease-associated pulmonary arterial hypertension. Arthritis Rheum 2013; 65: 3194-3201.

326 Gashouta MA, Humbert M, Hassoun PM. Update in systemic sclerosis-associated pulmonary arterial hypertension. Presse Med 2014; 43: e293-e304.

327 Coghlan JG, Denton CP, Gruenig E, et al. Evidence-based detection of pulmonary arterial hypertension in systemic sclerosis: the DETECT study. Ann Rheum Dis 2014; 73: 1340-1349.

328 Humbert M, Yaici A, de Groote P, et al. Screening for pulmonary arterial hypertension in patients with systemic sclerosis: clinical characteristics at diagnosis and long-term survival. Arthritis Rheum 2011; 63: 3522-3530.

329 Hao YJ, Jiang X, Zhou W, et al. Connective tissue disease-associated pulmonary arterial hypertension in Chinese patients. Eur Respir J 2014; 44: 963-972.

330 Hachulla E, Carpentier P, Gressin V, et al, ItinerAIR-Sclerodermie Study Investigators. Risk factors for death and the 3-year survival of patients with systemic sclerosis: the French ItinerAIR-Sclerodermie study. Rheumatology 2009; 48: 304-308.

331 Avouac J, Airo P, Meune C, et al. Prevalence of pulmonary hypertension in systemic sclerosis in European Caucasians and metaanalysis of 5 studies. J Rheumatol 2010; 37: 2290-2298.

332 Gunther S, Jaïs X, Maitre S, et al. Computed tomography findings of pulmonary venoocclusive disease in scleroderma patients presenting with precapillary pulmonary hypertension. Arthritis Rheum 2012; 64: 2995-3005.

333 Coghlan G. Does left heart disease cause most systemic sclerosis associated pulmonary hypertension? Eur Respir J 2013; 42: 888-890.

334 Humbert M, Sitbon O, Yaïci A, et al. Survival in incident and prevalent cohorts of patients with pulmonary arterial hypertension. Eur Respir J 2010; 36: 549-555.

335 Fisher MR, Mathai SC, Champion HC, et al. Clinical differences between idiopathic and scleroderma-related pulmonary hypertension. Arthritis Rheum 2006; 54: 3043-3050.

336 Launay D, Sitbon O, Hachulla E, et al. Survival in systemic sclerosis-associated pulmonary arterial hypertension in the modern management era. Ann Rheum Dis 2013; 72: 1940-1946.

337 O'Callaghan DS, Dorfmuller P, Jaï X, et al. Pulmonary veno-occlusive disease: the bete noire of pulmonary hypertension in connective tissue diseases? Presse Med 2011; 40: e65-e78.

338 Demling RH, Smith M, Gunther R, et al. Pulmonary injury and prostaglandin production during endotoxemia in conscious sheep. Am J Physiol 1981; 240: H348-H353.

339 Jais X, Launay D, Yaici A, et al. Immunosuppressive therapy in lupus- and mixed connective tissue disease-associated pulmonary arterial hypertension: a retrospective analysis of twenty-three cases. Arthritis Rheum 2008; 58: 521-531.

340 Avouac J, Wipff J, Kahan A, et al. Effects of oral treatments on exercise capacity in systemic sclerosis related pulmonary arterial hypertension: a meta-analysis of randomised controlled trials. Ann Rheum Dis 2008; 67: 808-814.

341 Launay D, Savale L, Berezne A, et al. Lung and heart-lung transplantation for systemic sclerosis patients. A monocentric experience of 13 patients, review of the literature and position paper of a multidisciplinary working group. Presse Med 2014; 43: e345-e363.

342 Hoeper MM, Krowka MJ, Strassburg CP. Portopulmonary hypertension and hepatopulmonary syndrome. Lancet 2004; 363: 1461-1468.

343 Zopey R, Susanto I, Barjaktarevic I, et al. Transition from hepatopulmonary syndrome to portopulmonary hypertension: a case series of 3 patients. Case Rep Pulmonol 2013; 2013: 561870.

344 Krowka MJ, Swanson KL, Frantz RP, et al. Portopulmonary hypertension: results from a 10-year screening algorithm. Hepatology 2006; 44: 1502-1510.

345 Kawut SM, Krowka MJ, Trotter JF, et al. Clinical risk factors for portopulmonary hypertension. Hepatology 2008; 48: 196-203.

346 Roberts KE, Fallon MB, Krowka MJ, et al. Genetic risk factors for portopulmonary hypertension in patients with advanced liver disease. Am J Respir Crit Care Med 2009; 179: 835-842. 
347 Krowka MJ, Miller DP, Barst RJ, et al. Portopulmonary hypertension: a report from the US-based REVEAL Registry. Chest 2012; 141: 906-915.

348 Le Pavec J, Souza R, Herve P, et al. Portopulmonary hypertension: survival and prognostic factors. Am J Respir Crit Care Med 2008; 178: 637-643.

349 Provencher S, Herve P, Jaï X, et al. Deleterious effects of beta-blockers on exercise capacity and hemodynamics in patients with portopulmonary hypertension. Gastroenterology 2006; 130: 120-126.

350 Hoeper MM, Seyfarth HJ, Hoeffken G, et al. Experience with inhaled iloprost and bosentan in portopulmonary hypertension. Eur Respir J 2007; 30: 1096-1102.

351 Halank M, Knudsen L, Seyfarth HJ, et al. Ambrisentan improves exercise capacity and symptoms in patients with portopulmonary hypertension. Z Gastroenterol 2011; 49: 1258-1262.

352 Reichenberger F, Voswinckel R, Steveling E, et al. Sildenafil treatment for portopulmonary hypertension. Eur Respir J 2006; 28: 563-567.

353 Kuo PC, Johnson LB, Plotkin JS, et al. Continuous intravenous infusion of epoprostenol for the treatment of portopulmonary hypertension. Transplantation 1997; 63: 604-606.

354 Swanson KL, Wiesner RH, Nyberg SL, et al. Survival in portopulmonary hypertension: Mayo Clinic experience categorized by treatment subgroups. Am J Transplant 2008; 8: 2445-2453.

355 Sakai T, Planinsic RM, Mathier MA, et al. Initial experience using continuous intravenous treprostinil to manage pulmonary arterial hypertension in patients with end-stage liver disease. Transpl Int 2009; 22: 554-561.

356 Savale L, Magnier R, Le Pavec J, et al. Efficacy, safety and pharmacokinetics of bosentan in portopulmonary hypertension. Eur Respir J 2013; 41: 96-103.

357 Humbert M, Segal ES, Kiely DG, et al. Results of European post-marketing surveillance of bosentan in pulmonary hypertension. Eur Respir J 2007; 30: 338-344.

Kia L, Shah SJ, Wang E, et al. Role of pretransplant echocardiographic evaluation in predicting outcomes following liver transplantation. Am J Transplant 2013; 13: 2395-2401.

359 Krowka MJ, Plevak DJ, Findlay JY, et al. Pulmonary hemodynamics and perioperative cardiopulmonary-related mortality in patients with portopulmonary hypertension undergoing liver transplantation. Liver Transplant 2000; 6: $443-450$.

360 Ashfaq M, Chinnakotla S, Rogers L, et al. The impact of treatment of portopulmonary hypertension on survival following liver transplantation. Am J Transplant 2007; 7: 1258-1264.

361 Raevens S, De Pauw M, Reyntjens K, et al. Oral vasodilator therapy in patients with moderate to severe portopulmonary hypertension as a bridge to liver transplantation. Eur J Gastroenterol Hepatol 2013; 25: 495-502. Plotkin JS, Kuo PC, Rubin LJ, et al. Successful use of chronic epoprostenol as a bridge to liver transplantation in severe portopulmonary hypertension. Transplantation 1998; 65: 457-459.

363 Austin MJ, McDougall NI, Wendon JA, et al. Safety and efficacy of combined use of sildenafil, bosentan, and iloprost before and after liver transplantation in severe portopulmonary hypertension. Liver Transplant 2008; 14: 287-291.

364 Grannas G, Neipp M, Hoeper MM, et al. Indications for and outcomes after combined lung and liver transplantation: a single-center experience on 13 consecutive cases. Transplantation 2008; 85: 524-531.

365 Robalino BD, Moodie DS. Association between primary pulmonary hypertension and portal hypertension: analysis of its pathophysiology and clinical, laboratory and hemodynamic manifestations. J Am Coll Cardiol 1991; 17: 492-498. Barbaro G, Lucchini A, Pellicelli AM, et al. Highly active antiretroviral therapy compared with HAART and bosentan in combination in patients with HIV-associated pulmonary hypertension. Heart 2006; 92: 1164-1166. Degano B. HIV-associated pulmonary arterial hypertension: survival and prognostic factors in the modern therapeutic era. AIDS 2010; 24: 67-75.

368 Opravil M, Pechère M, Speich R, et al. HIV-associated primary pulmonary hypertension. A case control study. Swiss HIV Cohort Study. Am J Respir Crit Care Med 1997; 155: 990-995.

369 Sitbon O, Lascoux-Combe C, Delfraissy JF, et al. Prevalence of HIV-related pulmonary arterial hypertension in the current antiretroviral therapy era. Am J Respir Crit Care Med 2008; 177: 108-113.

370 Sitbon O, Gressin V, Speich R, et al. Bosentan for the treatment of human immunodeficiency virus-associated pulmonary arterial hypertension. Am J Respir Crit Care Med 2004; 170: 1212-1217.

371 Montani D, Achouh L, Dorfmuller P, et al. Pulmonary veno-occlusive disease: clinical, functional, radiologic, and hemodynamic characteristics and outcome of 24 cases confirmed by histology. Medicine (Baltimore) 2008; 87: 220-233.

Lantuejoul S, Sheppard MN, Corrin B, et al. Pulmonary veno-occlusive disease and pulmonary capillary hemangiomatosis: a clinicopathologic study of 35 cases. Am J Surg Pathol 2006; 30: 850-857.

Humbert M, Maitre S, Capron F, et al. Pulmonary edema complicating continuous intravenous prostacyclin in pulmonary capillary hemangiomatosis. Am J Respir Crit Care Med 1998; 157: 1681-1685.

Mandel J, Mark EJ, Hales CA. Pulmonary veno-occlusive disease. Am I Respir Crit Care Med 2000; 162: 1964-1973.

375 Langleben D, Heneghan JM, Batten AP, et al. Familial pulmonary capillary hemangiomatosis resulting in primary pulmonary hypertension. Ann Intern Med 1988; 109: 106-109 [published erratum appears in Ann Intern Med 1988;109:439].

376 Best DH, Sumner KL, Austin ED, et al. EIF2AK4 mutations in pulmonary capillary hemangiomatosis. Chest 2014; 145: 231-236.

377 Seferian A, Helal B, Jaïs X, et al. Ventilation/perfusion lung scan in pulmonary veno-occlusive disease. Eur Respir J 2012; 40: 75-83.

378 Rabiller A, Jaïs X, Hamid A, et al. Occult alveolar haemorrhage in pulmonary veno-occlusive disease. Eur Respir J 2006; 27: 108-113.

379 Montani D, Jaïs X, Price LC, et al. Cautious epoprostenol therapy is a safe bridge to lung transplantation in pulmonary veno-occlusive disease. Eur Respir J 2009; 34: 1348-1356.

380 Fang JC, DeMarco T, Givertz MM, et al. World Health Organization Pulmonary Hypertension Group 2: pulmonary hypertension due to left heart disease in the adult-a summary statement from the Pulmonary Hypertension Council of the International Society for Heart and Lung Transplantation. J Heart Lung Transplant 2012; 31: 913-933. 
Ghio S, Gavazzi A, Campana C, et al. Independent and additive prognostic value of right ventricular systolic function and pulmonary artery pressure in patients with chronic heart failure. J Am Coll Cardiol 2001; 37: 183-188. Thenappan T, Shah SJ, Gomberg-Maitland M, et al. Clinical characteristics of pulmonary hypertension in patients with heart failure and preserved ejection fraction. Circ Heart Fail 2011; 4: 257-265.

Robbins IM, Newman JH, Johnson RF, et al. Association of the metabolic syndrome with pulmonary venous hypertension. Chest 2009; 136: 31-36.

Bursi F, McNallan SM, Redfield MM, et al. Pulmonary pressures and death in heart failure: a community study. $J$ Am Coll Cardiol 2012; 59: 222-231.

Gerges C, Gerges M, Lang MB, et al. Diastolic pulmonary vascular pressure gradient: a predictor of prognosis in "out-of-proportion" pulmonary hypertension. Chest 2013; 143: 758-766.

Moraes DL, Colucci WS, Givertz MM. Secondary pulmonary hypertension in chronic heart failure: the role of the endothelium in pathophysiology and management. Circulation 2000; 102: 1718-1723.

Rapp AH, Lange RA, Cigarroa JE, et al. Relation of pulmonary arterial diastolic and mean pulmonary arterial wedge pressures in patients with and without pulmonary hypertension. Am J Cardiol 2001; 88: 823-824.

Miller WL, Grill DE, Borlaug BA. Clinical features, hemodynamics, and outcomes of pulmonary hypertension due to chronic heart failure with reduced ejection fraction: pulmonary hypertension and heart failure. JACC Heart Fail 2013; 1: 290-299.

Tampakakis E, Leary PJ, Selby VN, et al. The diastolic pulmonary gradient does not predict survival in patients with pulmonary hypertension due to left heart disease. JACC Heart Fail 2015; 3: 9-16.

Pellegrini P, Rossi A, Pasotti M, et al. Prognostic relevance of pulmonary arterial compliance in patients with chronic heart failure. Chest 2014; 145: 1064-1070.

Chatterjee NA, Lewis GD. Characterization of pulmonary hypertension in heart failure using the diastolic pressure gradient: limitations of a solitary measurement. JACC Heart Fail 2015; 3: 17-21.

McMurray JJ, Adamopoulos S, Anker SD, et al. ESC Guidelines for the diagnosis and treatment of acute and chronic heart failure 2012: the Task Force for the Diagnosis and Treatment of Acute and Chronic Heart Failure 2012 of the European Society of Cardiology. Developed in collaboration with the Heart Failure Association (HFA) of the ESC. Eur Heart J 2012; 33: 1787-1847.

Khush KK, Tasissa G, Butler J, et al. Effect of pulmonary hypertension on clinical outcomes in advanced heart failure: analysis of the Evaluation Study of Congestive Heart Failure and Pulmonary Artery Catheterization Effectiveness (ESCAPE) database. Am Heart J 2009; 157: 1026-1034.

Patel ND, Weiss ES, Schaffer J, et al. Right heart dysfunction after left ventricular assist device implantation: a comparison of the pulsatile HeartMate I and axial-flow HeartMate II devices. Ann Thorac Surg 2008; 86: 832-840. Torre-Amione G, Southard RE, Loebe MM, et al. Reversal of secondary pulmonary hypertension by axial and pulsatile mechanical circulatory support. J Heart Lung Transplant 2010; 29: 195-200.

Barnett CF, DeMarco T. Pulmonary hypertension associated with left-sided heart disease. Heart Fail Clin 2012; 8: $447-459$.

Bonderman D, Ghio S, Felix SB, et al. Riociguat for patients with pulmonary hypertension caused by systolic left ventricular dysfunction: a phase IIb double-blind, randomized, placebo-controlled, dose-ranging hemodynamic study. Circulation 2013; 128: 502-511.

Oswald-Mammosser M, Weitzenblum E, Quoix E, et al. Prognostic factors in COPD patients receiving long-term oxygen therapy. Importance of pulmonary artery pressure. Chest 1995; 107: 1193-1198.

Kessler R, Faller M, Weitzenblum E, et al. "Natural history" of pulmonary hypertension in a series of 131 patients with chronic obstructive lung disease. Am J Respir Crit Care Med 2001; 164: 219-224.

Lettieri CJ, Nathan SD, Barnett SD, et al. Prevalence and outcomes of pulmonary arterial hypertension in advanced idiopathic pulmonary fibrosis. Chest 2006; 129: 746-752.

Thabut G, Dauriat G, Stern JB, et al. Pulmonary hemodynamics in advanced COPD candidates for lung volume reduction surgery or lung transplantation. Chest 2005; 127: 1531-1536.

Chaouat A, Bugnet AS, Kadaoui N, et al. Severe pulmonary hypertension and chronic obstructive pulmonary disease. Am J Respir Crit Care Med 2005; 172: 189-194.

Arcasoy SM, Christie JD, Ferrari VA, et al. Echocardiographic assessment of pulmonary hypertension in patients with advance lung disease. Am J Respir Crit Care Med 2003; 167: 735-740.

Fisher MR, Forfia PR, Chamera E, et al. Accuracy of Doppler echocardiography in the hemodynamic assessment of pulmonary hypertension. Am J Respir Crit Care Med 2009; 179: 615-621.

Nathan SD, Shlobin OA, Barnett SD, et al. Right ventricular systolic pressure by echocardiography as a predictor of pulmonary hypertension in idiopathic pulmonary fibrosis. Respir Med 2008; 102: 1305-1310.

Agusti AG, Barbera JA, Roca J, et al. Hypoxic pulmonary vasoconstriction and gas exchange during exercise in chronic obstructive pulmonary disease. Chest 1990; 97: 268-275.

Barbera JA, Roger N, Roca J, et al. Worsening of pulmonary gas exchange with nitric oxide inhalation in chronic obstructive pulmonary disease. Lancet 1996; 347: 436-440.

Simonneau G, Escourrou P, Duroux P, et al. Inhibition of hypoxic pulmonary vasoconstriction by nifedipine. $N$ Engl J Med 1981; 304: 1582-1585.

Morrell NW, Higham MA, Phillips PG, et al. Pilot study of losartan for pulmonary hypertension in chronic obstructive pulmonary disease. Respir Res 2005; 6: 88-95.

Saadjian A, Philip-Joet F, Paganelli F, et al. Long-term effects of cicletanine on secondary pulmonary hypertension. J Cardiovasc Pharmacol 1998; 31: 364-371.

Stolz D, Rasch H, Linka A, et al. A randomised, controlled trial of bosentan in severe COPD. Eur Respir J 2008; 32: 619-628.

Blanco I, Gimeno E, Munoz PA, et al. Hemodynamic and gas exchange effects of sildenafil in patients with chronic obstructive pulmonary disease and pulmonary hypertension. Am J Respir Crit Care Med 2010; 181: 270-278.

Zisman DA, Schwarz M, Anstrom KJ, et al. A controlled trial of sildenafil in advanced idiopathic pulmonary fibrosis. N Engl J Med 2010; 363: 620-628.

Blanco I, Santos S, Gea J, et al. Sildenafil to improve respiratory rehabilitation outcomes in COPD: a controlled trial. Eur Respir J 2013; 42: 982-992. 
Lederer DJ, Bartels MN, Schluger NW, et al. Sildenafil for chronic obstructive pulmonary disease: a randomized crossover trial. COPD 2012; 9: 268-275.

Goudie AR, Lipworth BJ, Hopkinson PJ, et al. Tadalafil in patients with chronic obstructive pulmonary disease: a randomised, double-blind, parallel-group, placebo-controlled trial. Lancet Respir Med 2014; 2: 293-300.

Lang IM, Pesavento R, Bonderman D, et al. Risk factors and basic mechanisms of chronic thromboembolic pulmonary hypertension: a current understanding. Eur Respir J 2013; 41: 462-468.

Guerin L, Couturaud F, Parent F, et al. Prevalence of chronic thromboembolic pulmonary hypertension after acute pulmonary embolism. Prevalence of CTEPH after pulmonary embolism. Thromb Haemost 2014; 112: $598-605$.

Pepke-Zaba J, Jansa P, Kim NH, et al. Chronic thromboembolic pulmonary hypertension: role of medical therapy. Eur Respir J 2013; 41: 985-990.

Madani MM, Wittine LM, Auger WR, et al. Chronic thromboembolic pulmonary hypertension in pediatric patients. J Thorac Cardiovasc Surg 2011; 141: 624-630.

Pepke-Zaba J, Hoeper MM, Humbert M. Chronic thromboembolic pulmonary hypertension: advances from bench to patient management. Eur Respir J 2013; 41: 8-9.

ang IM, Simonneau G, Pepke-Zaba JW, et al. Factors associated with diagnosis and operability of chronic thromboembolic pulmonary hypertension. A case-control study. Thromb Haemost 2013; 110: 83-91.

Lang IM, Plank C, Sadushi-Kolici R, et al. Imaging in pulmonary hypertension. JACC Cardiovasc Imaging 2010; 3: $1287-1295$

Hoeper MM, Mayer E, Simonneau G, et al. Chronic thromboembolic pulmonary hypertension. Circulation 2006; 113: 2011-2020.

AD, Swensen SJ, Hartman TE. Mosaic pattern of lung attenuation on CT scans: frequency among patients with pulmonary artery hypertension of different causes. Am J Roentgenol 1997; 169: 79-82.

Ley S, Ley-Zaporozhan J, Pitton MB, et al. Diagnostic performance of state-of-the-art imaging techniques for morphological assessment of vascular abnormalities in patients with chronic thromboembolic pulmonary hypertension (CTEPH). Eur Radiol 2012; 22: 607-616.

Sugiyama M, Fukuda T, Sanda Y, et al. Organized thrombus in pulmonary arteries in patients with chronic thromboembolic pulmonary hypertension; imaging with cone beam computed tomography. Jpn J Radiol 2014; 32: $375-382$

Shure D, Gregoratos G, Moser KM. Fiberoptic angioscopy: role in the diagnosis of chronic pulmonary arterial obstruction. Ann Intern Med 1985; 103: 844-850.

Skoro-Sajer N, Marta G, Gerges C, et al. Surgical specimens, haemodynamics and long-term outcomes after pulmonary endarterectomy. Thorax 2014; 69: 116-122.

Mayer E, Jenkins D, Lindner J, et al. Surgical management and outcome of patients with chronic thromboembolic pulmonary hypertension: results from an international prospective registry. J Thorac Cardiovasc Surg 2011; 141: 702-710.

Madani MM, Auger WR, Pretorius V, et al. Pulmonary endarterectomy: recent changes in a single institution's experience of more than 2,700 patients. Ann Thorac Surg 2012; 94: 97-103.

Jenkins D, Mayer E, Screaton N, et al. State-of-the-art chronic thromboembolic pulmonary hypertension diagnosis and management. Eur Respir Rev 2012; 21: 32-39.

Vuylsteke A, Sharples L, Charman G, et al. Circulatory arrest versus cerebral perfusion during pulmonary endarterectomy surgery (PEACOG): a randomised controlled trial. Lancet 2011; 378: 1379-1387.

Thistlethwaite PA, Madani MM, Kemp AD, et al. Venovenous extracorporeal life support after pulmonary endarterectomy: indications, techniques, and outcomes. Ann Thorac Surg 2006; 82: 2139-2145.

Berman M, Tsui S, Vuylsteke A, et al. Successful extracorporeal membrane oxygenation support after pulmonary thromboendarterectomy. Ann Thorac Surg 2008; 86: 1261-1267.

Mydin M, Berman M, Klein A, et al. Extracorporeal membrane oxygenation as a bridge to pulmonary endarterectomy. Ann Thorac Surg 2011; 92: e101-e103. pulmonary hypertension: a 1-year follow-up study. Eur Respir J 2006; 28: 138-143.

Skoro-Sajer N, Bonderman D, Wiesbauer F, et al. Treprostinil for severe inoperable chronic thromboembolic pulmonary hypertension. Thromb Haemost 2007; 5: 483-489.

Reichenberger F, Voswinckel R, Enke B, et al. Long-term treatment with sildenafil in chronic thromboembolic pulmonary hypertension. Eur Respir J 2007; 30: 922-927.

Jaïs X, D'Armini AM, Jansa P, et al. Bosentan for treatment of inoperable chronic thromboembolic pulmonary hypertension: BENEFiT (Bosentan Effects in iNopErable Forms of chronIc Thromboembolic pulmonary hypertension), a randomized, placebo-controlled trial. J Am Coll Cardiol 2008; 52: 2127-2134.

Ghofrani HA, D'Armini AM, Grimminger F, et al. Riociguat for the treatment of chronic thromboembolic pulmonary hypertension. N Engl J Med 2013; 369: 319-329.

Reesink HJ, Surie S, Kloek JJ, et al. Bosentan as a bridge to pulmonary endarterectomy for chronic thromboembolic pulmonary hypertension. J Thorac Cardiovasc Surg 2010; 139: 85-91.

Feinstein JA, Goldhaber SZ, Lock JE, et al. Balloon pulmonary angioplasty for treatment of chronic thromboembolic pulmonary hypertension. Circulation 2001; 103: 10-13.

Kataoka M, Inami T, Hayashida K, et al. Percutaneous transluminal pulmonary angioplasty for the treatment of chronic thromboembolic pulmonary hypertension. Circ Cardiovasc Interv 2012; 5: 756-762.

Mizoguchi H, Ogawa A, Munemasa M, et al. Refined balloon pulmonary angioplasty for inoperable patients with chronic thromboembolic pulmonary hypertension. Circ Cardiovasc Interv 2012; 5: 748-755.

Sugimura K, Fukumoto Y, Satoh K, et al. Percutaneous transluminal pulmonary angioplasty markedly improves pulmonary hemodynamics and long-term prognosis in patients with chronic thromboembolic pulmonary hypertension. Circ J 2012; 76: 485-488.

7 Inami $\mathrm{T}$, Kataoka $\mathrm{M}$, Shimura $\mathrm{N}$, et al. Pulmonary edema predictive scoring index (PEPSI), a new index to predict risk of reperfusion pulmonary edema and improvement of hemodynamics in percutaneous transluminal pulmonary angioplasty. JACC Cardiovasc Interv 2013; 6: 725-736.

Andreassen AK, Ragnarsson A, Gude E, et al. Balloon pulmonary angioplasty in patients with inoperable chronic thromboembolic pulmonary hypertension. Heart 2013; 99: 1415-1420. 
Rubin LJ, Hoeper MM, Klepetko W, et al. Current and future management of chronic thromboembolic pulmonary hypertension: from diagnosis to treatment responses. Proc Am Thorac Soc 2006; 3: 601-607.

450 Peacock A. Pulmonary hypertension due to chronic hypoxia. BMJ 1990; 300: 763.

451 Recommendations on the management of pulmonary hypertension in clinical practice. Heart 2001; 86(Suppl 1): I1-I13.

452 Consensus statement on the management of pulmonary hypertension in clinical practice in the UK and Ireland. Thorax 2008; 63(Suppl 2):ii1-ii41.

453 Barbera JA, Escribano P, Morales P, et al. [Standards of care in pulmonary hypertension. Consensus statement of the Spanish Society of Pulmonology and Thoracic Surgery (SEPAR) and the Spanish Society of Cardiology (SEC)]. Rev Esp Cardiol 2008; 61: 170-184.

454 Armstrong I, Rochnia N, Harries C, et al. The trajectory to diagnosis with pulmonary arterial hypertension: a qualitative study. BMJ Open 2012; 2: e000806.

455 Ghofrani HA, Distler O, Gerhardt F, et al. Treatment of pulmonary arterial hypertension (PAH): updated Recommendations of the Cologne Consensus Conference 2011. Int J Cardiol 2011;154(Suppl 1):S20-S33.

456 Vachiery JL, Gaine S. Challenges in the diagnosis and treatment of pulmonary arterial hypertension. Eur Respir Rev 2012; 21: 313-320. 